\title{
Quantum simulations and many-body physics with light
}

\author{
Changsuk Noh ${ }^{1,2}$ and Dimitris G. Angelakis ${ }^{1,3}$ \\ 1 Centre for Quantum Technologies, National University of Singapore, 3 Science Drive 2, Singapore 117543 \\ 2 Korea Institute for Advanced Study, 85 Hoegiro, Seoul 130722 and \\ 3 School of Electronic and Computer Engineering, \\ Technical University of Crete, Chania, Crete, Greece, 73100
}

(Dated: April 18, 2016)

\begin{abstract}
In this review we discuss the works in the area of quantum simulation and many-body physics with light, from the early proposals on equilibrium models to the more recent works in driven dissipative platforms. We start by describing the founding works on Jaynes-Cummings-Hubbard model and the corresponding photon-blockade induced Mott transitions and continue by discussing the proposals to simulate effective spin models and fractional quantum Hall states in coupled resonator arrays (CRAs). We also analyze the recent efforts to study out-of-equilibrium many-body effects using driven CRAs, including the predictions for photon fermionization and crystallization in driven rings of CRAs as well as other dynamical and transient phenomena. We try to summarise some of the relatively recent results predicting exotic phases such as super-solidity and Majorana like modes and then shift our attention to developments involving one dimensional nonlinear slow light setups. There the simulation of strongly correlated phases characterising Tonks-Girardeau gases, Luttinger liquids, and interacting relativistic fermionic models is described. We review the major theory results and also briefly outline recent developments in ongoing experimental efforts involving different platforms in circuit QED, photonic crystals and nanophotonic fibers interfaced with cold atoms.
\end{abstract}

\section{Contents}

\section{Introduction}

II. Equilibrium many-body effects with coupled resonator arrays

A. Photon-blockade induced Mott transitions and the Jaynes-Cummings-Hubbard Model

B. Mott and superfluid phases of light in the Jaynes-Cummings-Hubbard model

C. Effective spin models

D. Fractional Quantum Hall states of photons

III. Out-of-equilibrium physics in CRAs

A. Comparison between JCH and BH models 9

B. Fermionization and crystallization of photons and polaritons in $\mathrm{BH}$ and $\mathrm{JCH}$ models

1. Fermionization of photons

2. Crystallization of polaritons

C. Dynamical phenomena

1. Superfluid-insulator transition

2. Localization-delocalization transition of photons

D. Exotic phases out of equilibrium

1. Photon supersolid phase

2. Majorana-like modes

E. Further interesting works

F. Experimental implementations

IV. Simulating strongly correlated effects in 1D with photons in waveguides with EIT-based nonlinearities

A. Background

1. Stationary pulses of light

2. EIT nonlinearities with four-level atoms

B. Tonks-Girardeau gas of photons

9

9
C. Introducing an effective potential-Bose Hubbard and sine-Gordon models, and the 'pinning' transition

D. Bose-Einstein condensation of stationary-light polaritons

E. Two-component bosonic models: Luttinger liquids, spin-charge separation and Cooper pairing with photons

1. Luttinger liquid and spin-charge separation 25

2. BCS-BEC like crossover

F. Interacting relativistic theories with slow light 26

G. Experimental progress

H. Outlook: Rydberg polaritons

27

V. Summary and outlook

28

VI. Acknowledgements

29

References

29

\section{INTRODUCTION}

Quantum simulators offer a promising alternative when analytical and numerical methods fail in analyzing models with strong correlations. Strong correlations characterise effects in many different fields of physical science. To date, a collection of such effects in different areas ranging from condensed matter physics to relativistic quantum theories and nanotechnology have been simulated [1, 2], using different platforms such as trapped ions [3] and cold atoms in optical lattices [4].

In parallel with the progress in laser cooling that led to ultracold atoms and ion traps, the field of quantum nonlinear optics and cavity QED has seen great advances 
in the last two decades. This has motivated proposals to study many-body physics using strongly correlated photons (SCPs). Initially, coupled resonator arrays (CRAs) were considered where photons trapped in resonators were assumed to be interacting with real or artificial atoms. Pioneering works suggested the simulation of Mott transitions in CRAs assuming either i) two-level dopants leading to what is now known as the Jaynes-Cummings-Hubbard (JCH) model [5] or ii) fourlevel atoms with configurations used in electromagnetically induced transparency, giving rise to strongly interacting polaritons [6], and calculated the phase diagram of the JCH model using mean field theory [7]. These were followed soon after by a number of works investigating: beyond-mean-field effects using numerical methods [8], strongly correlated polaritons in photonic crystals [9], quantum state transfer 10 12, propagation of photonic or atomic excitations [13], quantum phase transition in the Tavis-Cummings lattice model [14, simulation of spin models [15 17, and the fractional quantum Hall effect 18 . We would like to mention here that there also exists a line of works which bring the exotic physics of single particle relativistic effects into the laboratory. These have also been categorised as quantum or optical simulations and range from numerous theoretical works involving the Dirac equation and associated phenomena including Zitterbewegung and Klein-tunneling [19]26 to experimental implementations of them in various platforms like the ones mentioned above [27, 28, and linear optics [29, 30]. In this review however, we will focus on simulations of strongly correlated phenomena with strongly interacting atom-photon systems.

These early proposals, and the numerous ones that followed studying the JCH system from different perspectives, marked the beginning of a novel direction in doing many-body physics and quantum simulations using lightmatter systems. To mention a few of the works without trying to be exhaustive, we refer the reader to: proposals for entanglement generation 31 34, polaritonic phase diagram studies using numerical and approximate analytic techniques 35 39], works on different connecting geometries 40, 41, two component models and the emergent solitonic behaviour [42, 43, the strong coupling theory for JCH 44, and applications in quantum information processing [4]. Implementations-wise, the coupled QED resonators found its ideal realization in circuit QED platforms 46, enjoying widely-tunable coupling strengths and low decoherence rates. Other technologies such as photonic crystal structures and open cavities configurations have also been explored [47, 48.

The next major development in the field was proposals for simulations of $1 \mathrm{D}$ continuous interacting models with photons and polaritons in quantum nonlinear optical setups. Here, light pulses trapped in hollow-core fibers interacting with cold atoms were shown to behave as a Tonks-Girardeau gas 49. This was soon extended to Luttinger liquids exhibiting spin charge separation [50]. More recently, a simulation of $1 \mathrm{D}$ interacting relativistic theories as described by the Thirring model was proposed, using polarised photons acting as effective fermions [51]. The ability to efficiently measure correlation functions of photonic states, the integrability of the proposed structures with other optical components on a chip, and the ability to operate at higher and even room temperatures, made quantum simulations with SCPs a quickly evolving field with many theoretical and experimental groups actively pursuing research.

We note here that the idea of using exciton polaritons (excitons coupled to photons in semiconductor materials) to realize quantum fluids has been developed in parallel, motivated by progress in controlling light-matter interactions in semiconductor structures [52. The interaction strength is typically weak in exciton-polariton systems but ways to enhance polariton-polariton interaction strength have been proposed (see for example [53]). Experimentally, the observation of Bose-Einstein condensation by Kasprzak et al. in 2006 [54 has stirred enormous interest in this system. The relationship between the paraxial propagation of a light beam in bulknonlinear media and the many-body quantum nonlinear Schrödinger equation has been pioneered by Lai and Haus 55. 56] and the possibility to use the latter type of system for quantum simulation has been recently addressed in [57]. Exciting developments in these fields have been extensively reviewed in [52], to which we refer the interested reader.

The aim of the current report is to review the evolution of SCPs as they have emerged from the early cavity QED approaches. The first part of this manuscript briefly reviews the early proposals on the simulations of Mott transition, the use of Mott state in quantum computing, quantum Heisenberg spin models, and the fractional quantum Hall effect in CRAs. The second part presents more recent results in the direction of out-of-equilibrium properties of lossy driven CRAs. We discuss similarities and differences between the JCH and the Bose-Hubbard systems and review various phenomena arising in either or both of these systems. Fermionization and crystallization of photons are first reviewed, which is followed by the works on dynamical signatures of superfluid-Mott transition and the so-called localization-delocalization transition. We then review more exotic effects such as photonic supersolid-like phases and Majorana-like modes and finish off with a survey of studies on smaller arrays and possible experimental implementations. The final part of this review is devoted to reviewing the recent progress in simulating strongly correlated one-dimensional systems using slow light in a hollow-core or tapered fiber coupled to an ensemble of cold atoms. In this part, we first review the proposal for realising a photonic Tonks-Girardeau gas, and then proceed to follow up works on simulating spin-charge separation and a photonic Luttinger liquid, the so-called pinning transition, BCS-BEC crossover, and interacting relativistic theories with photons. Although the focus in this part is on the main theoretical proposals, we also briefly refer to the most recent developments in 
ongoing experimental efforts in nonlinear fiber systems.

\section{EQUILIBRIUM MANY-BODY EFFECTS WITH COUPLED RESONATOR ARRAYS}

The early proposals on simulations of quantum manybody effects with coupled resonator arrays were partially motivated by: 1) advances in realising coupled resonator optical waveguides (CROWs) and 2) breakthroughs in achieving strong coupling between quantum emitters and single photons in various light-matter interfaces. CROWs were initially envisioned in photonic crystals structures [58 and were used mainly for classical microwave optics applications. These works were followed by studies in the optical frequencies for applications such as efficient guiding of light 59. and building Mach-Zehnder interferometers [60, 61]. The latter were extended, among others, to $2 \mathrm{D}$ CROW structures in 2D photonic crystals for tunable optical delay components and nonlinear optics 62. Strong coupling between single emitters and single photons was also implemented soon after both in semiconductor [63] and circuit QED platforms [64]. Motivated by these progress, and the race for coming up with efficient QIP implementations, the use of cavity QED physics in CROWs doped with quantum emitters was proposed for implementing photonic phase gates 65.

Next, the use of CRAs for quantum many-body simulations was proposed in an array of coupled QED resonators interacting strongly with single two level systems. Such a setup was envisioned as a photonic analogue of an optical lattice in atomic systems, that could allow both the trapping of photonic excitations and strong interactions between them. Taking advantage of the photon blockade effect-naturally arising when the atom is strongly coupled to the localized resonator photon 66. 67--it was shown that an insulator phase of the total (atomic+photonic or simply polaritonic) excitations was possible in an array of atom-resonator systems [5. In the same work, it was shown that, in parallel with optical lattices, one could mimic a quantum phase transition from the photonic insulator to the superfluid phase by varying the strength of the effective nonlinearity through the tuning of the atom-resonator detuning. Soon after, the complete phase diagram has been reproduced, first in 2D using mean field theory [7 and then in 1D using numerical methods based on DMRG techniques [8].

The Jaynes-Cummings-Hubbard (JCH) Hamiltonian as it came to be known, involves a combination of bosonic (photons) and spin excitations (the atoms). In general, the insulator-to-superfluid transition in this system is also accompanied by a transition in the nature of the excitations from polaritonic to photonic. Another important difference is that here quasiparticles are formed from hybrid light-matter excitations, rather than real physical particles such as atoms, and the former's internal structure makes the phase diagram extremely rich as we will discuss in detail later. In addition to the phase transi- tion, the possibility to simulate the dynamics of XY spin chains with individual-spin manipulation was proposed in the same work [5], using a mechanism different from that of optical lattices 68. In parallel with the above, the possibility to use 4-level atoms and external fields to simulate strongly interacting dynamics was proposed by Hartmann et al. [6. In this case, a time-dependent approach was employed, where the hopping/interaction ratio is varied in time such that the system initially starts deep in the Mott regime and ends up in the superfluid regime. A good agreement between the actually system and the $\mathrm{BH}$ model is found upon comparing the dynamics of the number fluctuations. This and subsequent works on simulating spin models using the four-level system approach have been reviewed in 69 .

\section{A. Photon-blockade induced Mott transitions and the Jaynes-Cummings-Hubbard Model}

In the following we describe in detail the initial proposal by Angelakis et al. [5] (first appeared in June 2006 and published in 2007), to implement the JCH model using two-level-system-doped CRAs in order to observe the Mott-superfluid transition of photons.

Consider a series of optical resonators coupled through direct photon hopping. The hopping could be generated through an overlap in the photonic modes as we assumed here or using mediating elements such as fibers [4]. The system is described by the Hamiltonian

$$
H=\sum_{k=1}^{N} \omega_{d} a_{k}^{\dagger} a_{k}-\sum_{k=1}^{N} J\left(a_{k}^{\dagger} a_{k+1}+\text { H.c. }\right)
$$

which corresponds to a series of coupled quantum harmonic oscillators. The photon frequency and hopping rate is $\omega_{d}$ and $J$ respectively and no nonlinearity is present yet. In order to simulate strongly correlated effects, interaction is necessary and the most natural way to induce it is to dope each resonator with a two level system. The latter could be an atom or a quantum dot or a superconducting qubit. Let us denote $|g\rangle_{k}$ and $|e\rangle_{k}$ as its ground and excited states at site $k$, respectively. The Hamiltonian describing the total system can be divided into three terms: $H_{\text {free }}$ the Hamiltonian for the free resonators and dopants, $H_{\text {int }}$ the Hamiltonian describing the local coupling of the photon and the dopant, and $H_{\text {hop }}$ for the photon hopping between cavities. Explicitly, these read

$$
\begin{aligned}
H_{\text {free }} & =\omega_{d} \sum_{k=1}^{N} a_{k}^{\dagger} a_{k}+\omega_{0} \sum_{k}|e\rangle_{k}\left\langle\left. e\right|_{k},\right. \\
H_{\text {int }} & =g \sum_{k=1}^{N}\left(a_{k}^{\dagger}|g\rangle_{k}\left\langle\left. e\right|_{k}+\text { H.c. }\right),\right. \\
H_{\text {hop }} & =-J \sum_{k=1}^{N}\left(a_{k}^{\dagger} a_{k+1}+\text { H.c. }\right),
\end{aligned}
$$




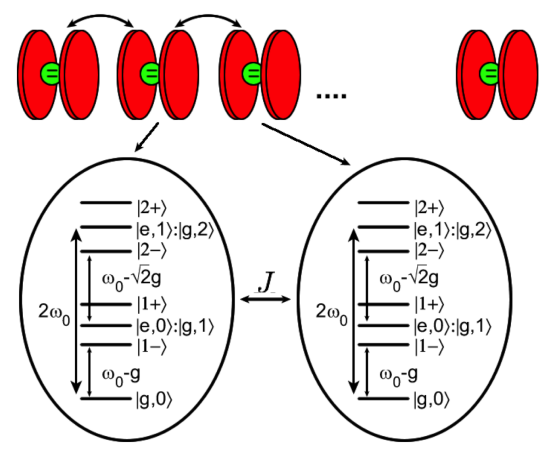

FIG. 1: (Top) Schematic representation of a series of cavities coupled through photon hopping. (bottom) The local energy structure in each atom-resonator system where the system's bare states are denoted as $\left\{|e, n-1\rangle_{k},|g, n\rangle_{k}\right\}$ and $|n \pm\rangle_{k}$ denotes the local eigenstates also known as dressed states. Reprinted from Angelakis et al. [5].

where $g$ is the atom-photon coupling strength. The local part of the Hamiltonian $H_{\text {free }}+H_{\text {int }}$ can be diagonalized in a basis of mixed photonic and atomic excitations. These excitations, known as dressed states or polaritons in quantum optics and condensed-matter communities respectively, involve a mixture of photonic and atomic excitations and are defined by annihilation operators $P_{k}^{( \pm, n)}=|g, 0\rangle_{k}\left\langle n \pm\left.\right|_{k}\right.$. The polaritonic states of the $k$ th atom-resonator system are given by $|n+\rangle_{k}=\left(\sin \theta_{n}|g, n\rangle_{k}+\cos \theta_{n}|e, n-1\rangle_{k}\right) / \sqrt{2}$ and $|n-\rangle_{k}=\left(\cos \theta_{n}|g, n\rangle_{k}-\sin \theta_{n}|e, n-1\rangle_{k}\right) / \sqrt{2}$, where $|n\rangle_{k}$ denotes the $n$-photon Fock state in the $k$ th resonator, $\tan 2 \theta_{n}=2 g \sqrt{n} / \Delta$, and $\Delta=\omega_{0}-\omega_{d}$ is the atom-light detuning. These polaritons have the energies $E_{n}^{ \pm}=n \omega_{d}+\Delta / 2 \pm \sqrt{n g^{2}+(\Delta / 2)^{2}}$ and are also eigenstates of the sum of the photonic and atomic excitation operators $\mathcal{N}_{k}=a_{k}^{\dagger} a_{k}+|e\rangle\left\langle\left. e\right|_{k}\right.$ with the eigenvalue $n$ (figure 1).

Studying the energy eigenstates of the system consistent with a given number of net excitations per site (or filling factor), it was shown that the ground state of the system reaches a Mott state of the polaritons for integer values of the filling factor. In contrast to the optical lattice systems where the lattice depth is tuned, here the detuning $\Delta=\omega_{0}-\omega_{d}$ is varied. The latter results in further shifts of the dressed states from the bare resonances, providing an effective control over effective nonlinearity. The hopping strength $J$ is usually fixed by the fabrication process and is harder to change experimentally. To see how the Mott state arises, it is convenient to rewrite the Hamiltonian in terms of the polaritonic operators (assuming $\Delta=0)$ :

$$
\begin{aligned}
H & =\sum_{k=1}^{N} \sum_{n=1}^{\infty}\left[n\left(\omega_{d}-g\right) P_{k}^{(-, n) \dagger} P_{k}^{(-, n)}\right. \\
& +n\left(\omega_{d}+g\right) P_{k}^{(+, n) \dagger} P_{k}^{(+, n)}+g(n-\sqrt{n}) P_{k}^{(-, n) \dagger} P_{k}^{(-, n)}
\end{aligned}
$$

$$
\begin{aligned}
& \left.+g(\sqrt{n}-n) P_{k}^{(+, n) \dagger} P_{k}^{(+, n)}\right] \\
& -J \sum_{k=1}^{N}\left(a_{k}^{\dagger} a_{k+1}+\text { H.c. }\right) . .
\end{aligned}
$$

Remember that the lower of the two energy eigenstates of the local system with a given number of excitations $\eta$ is the '-' dressed state $|\eta-\rangle$. Therefore, assuming the regime $J n<<g \sqrt{n}<<\omega_{d}$, one needs only consider the first, third and last terms of the above Hamiltonian for determining the low-lying energy eigenstates of the whole system. The first line corresponds to a linear spectrum, equivalent to that of a harmonic oscillator of frequency $\omega_{d}-g$. If only that part were present in the Hamiltonian, then it would not cost any extra energy to add an excitation (of frequency $\omega_{d}-g$ ) to a site already filled with one or more excitations, as opposed to an empty site.

The third term, on the contrary, provides an effective "on-site" photonic repulsion, leading to the formation of the Mott state of polaritons as the ground state of the system for a commensurate filling. Reducing the strength of the effective nonlinearity (or the photonic repulsion) through detuning, one can then drive the system to the superfluid (SF) regime. This could be done, for example, by Stark shifting the atomic transitions from the resonator by an external field. The shift being inversely proportional to the detuning, the new detuned polaritons are not as well separated as before, and in the case of large detuning, it costs little extra energy to add excitations (excite transitions to higher polaritons) in a single site. As a result, the system moves towards the SF regime with increasing detuning. Note here that the mixed nature of the polaritons could in principle allow for excitations that are mostly photonic, resulting in a photonic Mott state 70.

\section{B. Mott and superfluid phases of light in the Jaynes-Cummings-Hubbard model}

Beyond the qualitative description reproduced above, an exact numerical simulation was performed in [5], for a different number of sites (from three to seven) using the Hamiltonian $H=H_{\text {free }}+H_{\text {int }}+H_{\text {hop }}$. Note that such finite-size calculations are often employed to study the physics of the transition between Mott and superfluid phases [71] because mean-field theories in low dimensions are not guaranteed to work. We also note here that numerical simulations of the $\mathrm{JCH}$ Hamiltonian are more computationally costly than the ones for the BH Hamiltonian due to the existence of both bosonic (photon) and atomic operators in every site.

As discussed in [5], an appropriate order parameter in the $\mathrm{JCH}$ lattice is the variance of the polariton number per site, as the usual order parameter employed in other cases-like the expectation value of the annihilation operator in the BH model-is identically zero in a closed system. The variance $\operatorname{var}\left(\mathcal{N}_{k}\right)$ is plotted in figure 2 as 


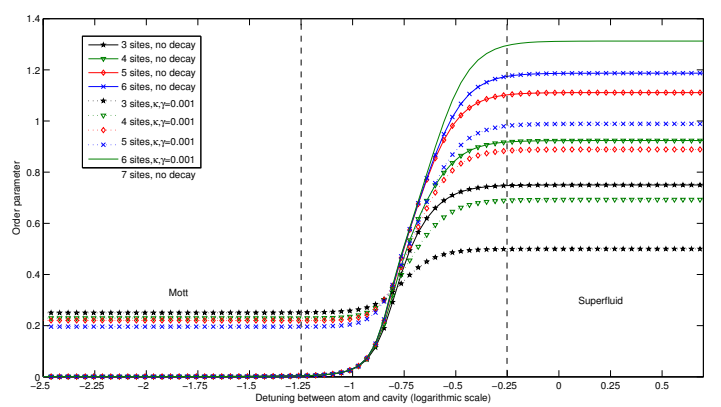

FIG. 2: The order parameter $\left(\operatorname{var}\left(\mathcal{N}_{k}\right)\right)$ as a function of the detuning $\Delta$ for 3-7 sites, with and without atomic dissipation and resonator leakage. Close to the atom-photon resonance $\left(0 \leq \Delta / g \leq 10^{-1}\right)$, where the effective on-site repulsion is maximum (and much larger than the hopping rate $A(=J)$ ), the system is forced into a polaritonic Fock state with the same number of excitations per site, i.e., the Mott insulator state. Increasing the detuning by applying external fields and inducing Stark shifts $(\Delta \geq g)$ weakens the repulsion, leading to the appearance of different coherent superpositions of excitations per site, i.e., a photonic superfluid state. The increase in the system size leads to a sharper transition as expected. Reprinted from Angelakis et al. [5].

a function of $\log _{10}(\Delta / g)$ for a filling factor of one net excitation per site. For this plot, we have taken the parameter ratio $g / J=10^{2}\left(g / J=10^{1}\right.$ gives quantitatively similar results), with $\Delta$ varying from $\sim 10^{-3} g$ to $\sim g$ and $\omega_{d}, \omega_{0} \sim 10^{4} \mathrm{~g}$. The order parameter for dissipationless atoms and cavities is displayed as solid curves and corresponding curves in the presence of a resonator dissipation and atomic spontaneous emission of $\sim 0.01 \mathrm{~g}$ are depicted with dotted lines.

In figure 3 the quantum dynamics characterizing the excitation probability of the middle site in a chain of three cavities for different regimes are calculated assuming the middle resonator is initially empty. We observe the blockade of higher than one excitation in the limit of resonant interaction (where the Mott phase is expected) as discussed in figure 2 .

The phase diagram of the $\mathrm{JCH}$ model has first been studied in 2D using mean field theory [7] and in 1D using a numerical method based on DMRG techniques 8. Figure 4(top) shows slices of the superfluid order parameter $\psi=\left\langle a_{i}\right\rangle$ as a function of the photon hopping and the chemical potential for different values of atom-photon detuning. A triangular lattice was assumed and the usual decoupling approximation $a_{i}^{\dagger} a_{j}=$ $\left\langle a_{i}^{\dagger}\right\rangle a_{j}+a_{i}^{\dagger}\left\langle a_{j}\right\rangle+\left\langle a_{i}^{\dagger}\right\rangle\left\langle a_{j}\right\rangle$ was used [7]. The bottom panels of figure 4 show the numerically-calculated phase diagram (top row) and the compressibility $(\kappa=\partial \rho / \partial \mu, \rho=$ filling factor) (bottom row) as functions of the hopping strength for a $1 \mathrm{D}$ chain.

In both figures the corresponding Mott and SF phases are explored by changing the hopping strength and the effective photonic chemical potential (the free energy term

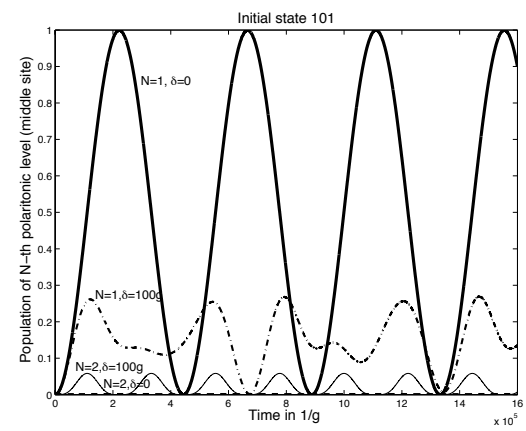

FIG. 3: The probability of exciting the polaritons corresponding to one (thick solid line) or two (thick dashed line) excitations for the middle resonator which is taken initially to be empty (in the resonant regime). In this case, as we have started with fewer polaritons than the number of cavities, oscillations occur for the single polariton excitation. The double excitation manifold, however, is never occupied due to the blockade effect (thick dashed line). For the detuned case, hopping of more than one polaritons is allowed (SF regime) which is evident as the higher polaritonic manifolds are being populated now (dot-dashed line and thin solid line). Reprinted from Angelakis et al. [5].

in the JCH Hamiltonian), following the standard treatment of quantum phase transitions. We note that in CRA experiments the hopping strength is in general not tunable but fixed by the fabrication process. In these systems, it is easier to control the effective repulsion (the photon blockade), as discussed in the previous section. Nevertheless, the phase diagrams are useful in showing the existence of Mott lobes in this photonic system and how they depend on system parameters. These works have been supplemented using the variational cluster approach, where on top of the phase diagram, singleparticle spectra and finite temperature effects have been investigated 35. Quantum Monte-Carlo method has also been employed to find various properties of the JCHtype systems [36, 72 74].

An application of the Mott regime for implementing quantum information processing (QIP): The typical implementation of cluster state quantum computing requires initializing all qubits in a 2D lattice in the $|+\rangle=$ $(|0\rangle+|1\rangle) / \sqrt{2}$ state and then performing controlled-phase gates $(C P)$ between all nearest-neighbours [75]. In the resonator array system the first proposal to create cluster states utilized the Mott state and the resulting XY dynamics [16. The ground state $|g, 0\rangle$ and the first excited state $|1-\rangle$ of the combined atom-photon (polaritonic) system in each site was used as a qubit as depicted in figure 5

The need to disable the evolution was realized by combining the system's natural dynamics with a protocol where some of the available physical qubits are used as gate "mediators" and the rest as the logical qubits. The mediators can be turned on and off on demand by tuning the atoms in the corresponding sites on and off resonance 

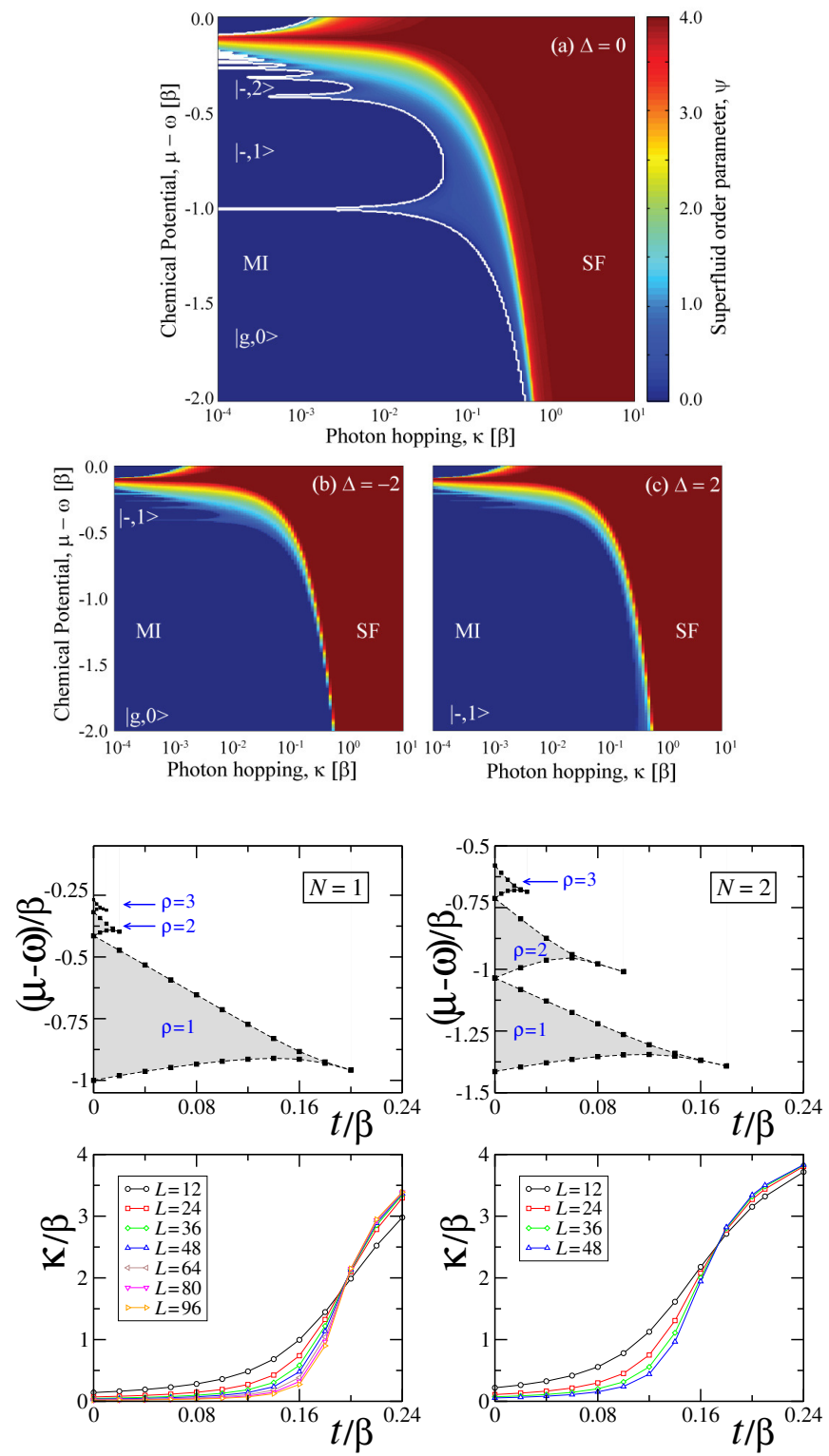

FIG. 4: (Top) The phases diagram of a 2D JCH system calculated using mean field theory for three different values of atom-photon detuning $\Delta=0$ (a), $-2 g$ (b), and $2 g$ (c). $\mu$ is the chemical potential introduced separately to the Hamiltonian, $\kappa$ is the hopping strength $(=J), \beta$ is the atom-photon coupling strength $(=g)$, and $\omega$ is the bare resonator frequency $\left(=\omega_{d}\right)$ 7]. Reprinted with permission from Greentree et al. 7]. (Bottom, top row) The phase diagram of a 1D JCH array, calculated using DMRG simulations. $\mu$ is the chemical potential, $\beta$ is the atom-photon coupling strength $(=g), t$ is the hopping strength $(=J)$, and $\rho$ is the filling factor in the Mott phase. (Bottom, bottom row) The compressibility $\kappa$ as a function of hopping strength, for different system sizes $L$. $N=1$ (left column) and $N=2$ (right column) denotes the number of atoms inside each resonator [8]. Reprinted with permission from Rossini and Fazio [8].

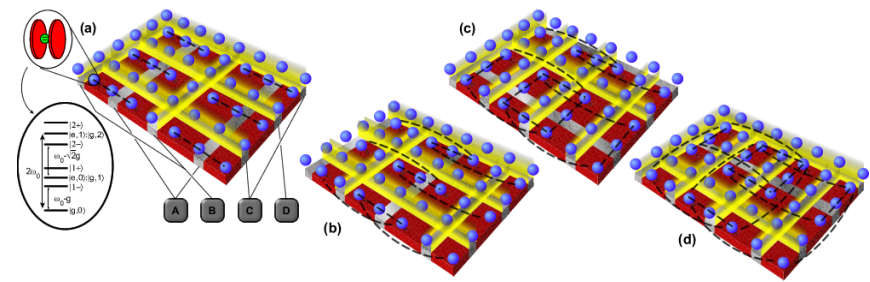

FIG. 5: A 2D array of atom-resonator systems in the Mott state can be used to implement cluster state quantum computing, utilizing the natural evolution, which in this case is of an XY spin type, and applying external global gates to switch on and off the dynamics. by properly applying local external fields, utilizing the fact that the cavities can be well separated. Reprinted from Angelakis and Kay [16].

from their cavities. This is done by Stark shifting them through the application of an external field (gates A, B, C $\mathrm{D}$ in figure 5), effectively shifting the site frequencies and inhibiting photon hopping, thereby isolating each logical qubit. Soon after there have also been other approaches focusing on the generation of the Ising interaction [76, 77 and also towards the simulation of a family of effective spin models in CRAs [15, 41, 78, 80. These will be analyzed in the next section.

\section{Effective spin models}

Quantum spin models have played a crucial role as basic models accounting for magnetic and thermodynamic properties of many-body systems. In implementations involving arrays of Josephson junctions 81 or quantum dots [82, the spin-chain Hamiltonian naturally emerges from the spin-like coupling between qubits, albeit with limited control over the coupling constants. In optical lattice simulators, perturbative dynamics with respect to the Mott-insulator state allows for an effective spin-chain Hamiltonian 68, 83. This direction has the advantage that the spin-coupling constants can be optically controlled to a great extent. In CRAs, a spin is represented by either polariton states or hyperfine ground levels of the embedded emitter and the system was shown to generate an effective Heisenberg model with optically tunable interactions

$$
H_{e f f}=-\sum_{\langle j, k\rangle} \lambda_{z} \sigma_{j}^{Z} \sigma_{k}^{Z}+\lambda_{x} \sigma_{j}^{X} \sigma_{k}^{X}+\lambda_{y} \sigma_{j}^{y} \sigma_{k}^{y}+h \sum_{j} \sigma_{j}^{Z},
$$

where the angled brackets denote the sum over nearest neighbours. We will use the same notation throughout this review.

In the work [78, an array of cavities was assumed on the vertices of a 2D lattice (note that any array is in principle possible) as depicted in figure 6(a). Each resonator is doped with a single system (which we refer to as an atom), whose energy level structure comprises of a ground state $|g\rangle$ and two degenerate excited states $|A\rangle$ 


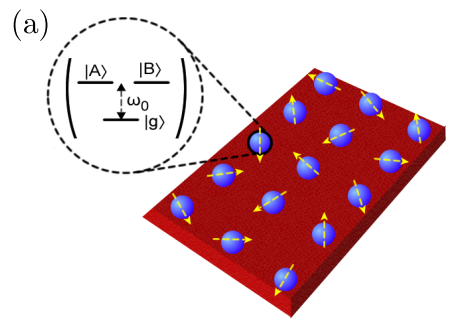

(b)

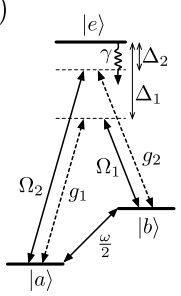

FIG. 6: (a) Two-dimensional CRA for simulating Heisenberg spin models. Each resonator is doped with a V-type atom which is coupled to two orthogonal modes 78. Reprinted with permission from Kay and Angelakis 78. (b) The atomic level structure and optical transitions involved in order to simulate higher-spin Heisenberg models 84. Reprinted from Cho et al. 84.

and $|B\rangle$. Within each lattice site, the Hamiltonian takes the form

$$
\begin{aligned}
H_{0}= & \omega_{0}\left(a_{i}^{\dagger} a_{i}+b_{i}^{\dagger} b_{i}+|A\rangle\left\langle\left. A\right|_{i}+\mid B\right\rangle\left\langle\left. B\right|_{i}\right)\right. \\
& +g\left(|A\rangle\left\langle\left. g\right|_{i} \otimes a_{i}+\mid g\right\rangle\left\langle\left. A\right|_{i} \otimes a_{i}^{\dagger}\right)\right. \\
& +g\left(|B\rangle\left\langle\left. g\right|_{i} \otimes b_{i}+\mid g\right\rangle\left\langle\left. B\right|_{i} \otimes b_{i}^{\dagger}\right)\right.
\end{aligned}
$$

where $a_{i}^{\dagger}$ and $b_{i}^{\dagger}$ create photons of orthogonal polarizations at site $i$ and the atomic levels and the resonator modes are assumed to be on resonance. The strength of the coupling between the resonator and the atom is denoted by $g$. Coupling between nearest-neighbour resonators is described by the Hamiltonian

$$
H_{h o p}=\sum_{\langle i, j\rangle} J_{a}\left(a_{i}^{\dagger} a_{j}+\text { h.c. }\right)+J_{b}\left(b_{i}^{\dagger} b_{j}+\text { h.c. }\right) .
$$

Assuming strong atom-photon interaction regime, $g \gg$ $J_{a, b}$, and restricting to the unit filling fraction, such that one excitation per site is expected, one obtains an effective Hamiltonian (6) with

$$
\begin{aligned}
h & =\frac{5}{8 g}\left(J_{a}^{2}-J_{b}^{2}\right), \quad \lambda_{z}=\frac{9}{32 g}\left(J_{a}^{2}+J_{b}^{2}\right), \\
\lambda_{x} & =\lambda_{y}=\frac{9 J_{a} J_{b}}{16 g} .
\end{aligned}
$$

Note that we have ignored the constant term $\kappa=$ $\frac{31}{32 g}\left(J_{a}^{2}+J_{b}^{2}\right)$. The local magnetic fields can be manipulated by applying local Stark fields, thereby leaving an XXZ Hamiltonian where the coefficients $\lambda_{z}$ and $\lambda_{x}$ are independently tunable (at device-manufacture stage). This approach allows a stronger spin-spin coupling than an alternative one in [15], but lacks the optical control of the coupling. The latter exhibits greater optical controllability, but relies on rapid switching of optical pulses and the consequent Trotter expansion, which unavoidably introduces additional errors and makes the implementation more difficult.
The question of simulating chains of higher spins, which may have a completely different phase diagram, was dealt with in 84. In this proposal, a fixed number of atoms are confined in each resonator with collectively applied constant laser fields, and is in a regime where both atomic and resonator excitations are suppressed (figure 6(b)). It was thus shown that as well as optically controlling the effective spin-chain Hamiltonian, it is also possible to engineer the magnitude of the spin.

\section{Fractional Quantum Hall states of photons}

Since its first discovery in a semiconductor heterostructure in the early 1980s 85], systems exhibiting FQHE has now become routinely available in laboratories. In a different context, the achievement of trapping ultracold atomic gases in a strongly correlated regime has prompted an interest in mimicking various condensed matter systems with trapped atoms, thereby allowing one to tackle such complex systems in unprecedented ways [86. The great advantage of this approach is that such a tailored system is highly controllable at the microscopic level and is close to idealized quantum models used in condensed matter systems. In the context of FQHE, a 2D atomic gas is trapped in a harmonic potential [87, 88, or in an optical lattice [89, 90. Since the atoms in consideration have no real charge, the magnetic field is simulated artificially. For instance, it can be done by rapidly rotating the harmonic trap [87, by exploiting electromagnetically induced transparency [88, or by modulating the optical lattice potential [89, 90 .

The first proposal to realize the fractional quantum Hall system with strongly correlated photons was based on an optical manipulation of atomic internal states and inter-resonator hopping of virtually excited photons in a two-dimensional array of coupled cavities [18]. It was shown that the local addressability of coupled resonator systems allows one to simulate any system of hard-core bosons on a lattice in the presence of an arbitrary Abelian vector potential. The proposal is based on the realization of the Heisenberg spin model introduced earlier and is depicted in figure 7. To facilitate a spatially varying gauge potential, an asymmetry in the resonator modes is assumed, where two orthogonal resonator modes along the $x$ and $y$ directions have different resonant frequencies (this asymmetry is not an essential requirement as explained in [18]). Realizing this geometry would be viable in several promising platforms, such as photonic bandgap microcavities and superconducting microwave cavities. The frequency difference between the two modes is assumed to be much larger than the atom-resonator coupling rates. In this way, either direction of the spin exchange can be accessed individually.

Similar works with different approaches and platforms within CRA have appeared since. Ways to introduce synthetic gauge fields-allowing one to break the timereversal symmetry which is crucial in quantum Hall 
physics-have been devised in circuit QED [91, 92] and solid state cavities [93. A photonic version of the quantum spin Hall system, which does not break the timereversal symmetry, has also been proposed [94] and experimentally realized 95. Interacting versions realizing fractional quantum Hall-type physics of photons were proposed in Kerr-nonlinear cavities [96] and the $\mathrm{JCH}$ cavities 97 and signatures of fractional quantum Hall physics in a non-equilibrium driven-dissipative scenario have been investigated [98.

(a)

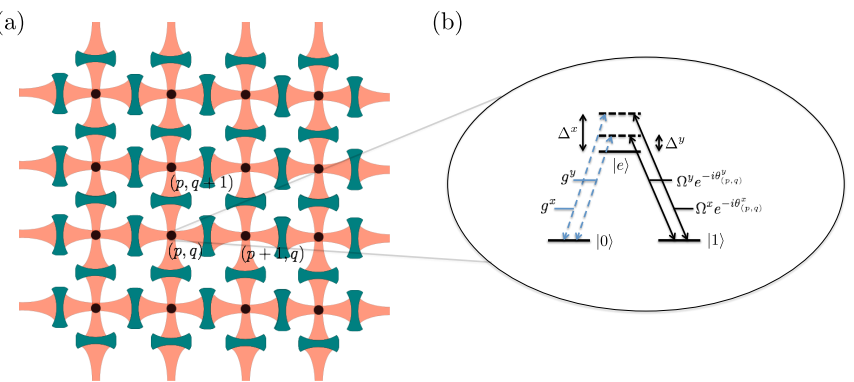

FIG. 7: (a) Schematic representation of a 2D CRA which simulates a bosonic FQH states. Each site supports two orthogonal nondegenerate resonator modes that are coupled with a single atom. (b) Involved atomic levels and transitions. The resonator mode $x(y)$ is coupled to the atomic transition $|e\rangle\langle 0|$ with coupling strengths $g^{x}\left(g^{y}\right)$ and detun$\operatorname{ing} \Delta^{x}\left(\Delta^{y}\right)$. The transition $|e\rangle\langle 1|$ is driven by site-dependent laser fields with Rabi frequencies $\Omega^{x} e^{-i \theta_{(p, q)}^{x}}$ and $\Omega^{y} e^{-i \theta_{(p, q)}^{y}}$. The subscripts denote the position of the site. Adapted with permission from 99 .

Here we will take a closer look at the first proposal. But first, to set the stage, let us very briefly describe the model Hamiltonian and its ground state wave function that captures the physics of FQHE in its fermionic counterpart.

A system of bosonic particles confined in a 2D square lattice in the presence of an artificial perpendicular magnetic field $B$ is described by the Hamiltonian

$$
H_{0}=-t \sum_{p, q}\left(c_{p+1, q}^{\dagger} c_{p, q} e^{-i 2 \pi \alpha q}+c_{p, q+1}^{\dagger} c_{p, q}+\text { h.c. }\right),
$$

where the positions of lattice sites are represented by $a(p \hat{x}+q \hat{y})$ with $p$ and $q$ being integers and $\alpha$ is the spacing of the lattice. Here, $\alpha \equiv B a^{2} / \Phi_{0}$, the number of magnetic flux quanta through a lattice cell, plays a crucial role in characterizing the energy spectrum, whose self-similar structure is known as the Hofstadter butterfly. In the hardcore (maximum one boson per site) and continuum limit $(\alpha \ll 1)$ the ground state is accurately described by the Laughlin state

$$
\Psi_{m}\left(\left\{z_{j}\right\}\right)=e^{-\frac{1}{4} \sum_{j}\left|z_{j}\right|^{2}} \prod_{j<k}\left(z_{j}-z_{k}\right)^{m},
$$

where $z_{j}=x_{j}+i y_{j}$ and the filling factor $\nu=1 / m$ corresponds to the ratio of the number of bosons to the number of magnetic flux quanta.

Assume now a two-dimensional array of coupled cavities, each confining a single atom with two ground levels as in figure 7(b). Each resonator has two modes along the $x$ and $y$ directions, whose annihilation operators will be denoted by $a^{x}$ and $a^{y}$. The atom interacts with these resonator modes with coupling rates $g^{x}$ and $g^{y}$, and with detunings $\Delta^{x}$ and $\Delta^{y}$, respectively. Furthermore, to induce site-dependent hopping, two classical fields with (complex) Rabi frequencies $\Omega^{x} e^{-i \theta_{(p, q)}^{x}}$ and $\Omega^{y} e^{-i \theta_{(p, q)}^{y}}$ are applied. In the rotating frame, the Hamiltonian for the system reads

$$
\begin{aligned}
H= & \sum_{\substack{\mu=x, y \\
j=(p, q)}}\left[g^{\mu} e^{-i \Delta^{\mu} t} a_{j}^{\mu}(|e\rangle\langle 0|)_{j}\right. \\
& \left.+\Omega^{\mu} e^{-i \theta_{j}^{\mu}} e^{-i \Delta^{\mu} t}(|e\rangle\langle 1|)_{j}+\text { h.c. }\right] \\
- & \sum_{p, q}\left(J^{x} a_{p+1, q}^{x \dagger} a_{p, q}^{x}+J^{y} a_{p, q+1}^{y \dagger} a_{p, q}^{y}+\text { h.c. }\right),
\end{aligned}
$$

where $J^{x}\left(J^{y}\right)$ denotes the inter-resonator hopping rate of the photon along the $x(y)$ direction. We assume $\Delta^{x}-$ $\Delta^{y} \gg g^{x}, g^{y}$, as mentioned above, and furthermore that $\Delta^{\mu} \gg g^{\mu} \gg \Omega^{\mu}, J^{\mu}$.

In this strong coupling regime, the atomic excitation is suppressed and one can perform adiabatic elimination to obtain an effective Hamiltonian

$$
\begin{aligned}
H & =\sum_{\substack{\mu=x, y \\
j=(p, q)}}\left[\delta^{\mu} a_{j}^{\mu \dagger} a_{j}^{\mu}(|0\rangle\langle 0|)_{j}+\omega^{\mu}\left(e^{i \theta_{j}^{\mu}} a_{j}^{\mu} \sigma_{j}^{+}+\text {h.c. }\right)\right] \\
& -\sum_{p, q}\left(J^{x} a_{p+1, q}^{x \dagger} a_{p, q}^{x}+J^{y} a_{p, q+1}^{y \dagger} a_{p, q}^{y}+\text { h.c. }\right),
\end{aligned}
$$

where $\delta^{\mu}=\left(g^{\mu}\right)^{2} / \Delta^{\mu}, \omega^{\mu}=g^{\mu} \Omega^{\mu} / \Delta^{\mu}$, and $\sigma^{+}=|1\rangle\langle 0|$. Here, we have ignored the ac Stark shift induced by the classical fields, which is negligible in the assumed regime (it may also be compensated by other lasers). We further assume $\delta^{\mu} \gg J^{\mu} \gg \omega^{\mu}$, which can be satisfied, along with the above conditions, when

$$
g^{\mu} / \Delta^{\mu} \gg J^{\mu} / g^{\mu} \gg \Omega^{\mu} / \Delta^{\mu} .
$$

Now the resonator photon is suppressed and an adiabatic elimination can be applied once more. Keeping the terms up to the third order and taking only the submanifold with no photons, the effective Hamiltonian further simplifies to

$$
\begin{aligned}
H & =-t \sum_{p, q}\left[\sigma_{p+1, q}^{+} \sigma_{p, q}^{-} e^{i\left(\theta_{p+1, q}^{x}-\theta_{p, q}^{x}\right)}\right. \\
& \left.+\sigma_{p, q+1}^{+} \sigma_{p, q}^{-} e^{i\left(\theta_{p, q+1}^{y}-\theta_{p, q}^{y}\right)}+\text { h.c. }\right],
\end{aligned}
$$

where $\delta^{\mu}, \epsilon^{\mu}, \omega^{\mu}$, and $J^{\mu}$ are chosen to yield

$$
t=J^{x}\left(\frac{\omega^{x}}{\delta^{x}}\right)^{2}=J^{y}\left(\frac{\omega^{y}}{\delta^{y}}\right)^{2} .
$$


It is easy to see that this Hamiltonian reduces to the Hamiltonian $H_{0}$ in (8) in the hardcore bosonic limit, if we adjust the phases of the classical fields such that $\theta_{p, q}^{x}=$ $-p q \cdot 2 \pi \alpha$ and $\theta_{p, q}^{y}=0$. The ground state of the Hamiltonian (13) is expected to be described faithfully by the Laughlin wavefunction (9), modulo changes due to the difference in gauge and boundary conditions, which can be calculated analytically [100]. For a 4 by 4 lattice with $\alpha=1 / 4, m=2$, the fidelity between the numerically calculated ground state of the ideal Hamiltonian and this modified Laughlin state (with $m=2$ ) was shown to be excellent indeed $(\approx 0.989)$. The numerical ground states of the effective Hamiltonian (11) for $\delta^{\nu} / 10=10 \omega^{\mu}=J^{\mu}$, corresponding to $\Delta^{\mu} / 1000=g^{\mu} / 100=\Omega^{\mu}=J^{\mu}$, has the fidelity of 0.976 . The ground state can be prepared adiabatically by exciting two atoms at different sites initially without the laser fields $(t=0)$ and then delocalizing these excitations via adiabatically tuning up the effective hopping rate. The quasi-excitations can also be generated via local tuning of the laser fields to mimic an infinitely thin solenoid, around which the excitations form. More details on these issues can be found in [18, 99].

\section{OUT-OF-EQUILIBRIUM PHYSICS IN CRAS}

All the works we have reviewed so far were on equilibrium physics of CRAs. However, as photonic systems are intrinsically dissipative, they are naturally operated in a non-equilibrium setting where some sort of external pumping is often introduced. This is evident for example in recent experiments on BECs of exciton-polaritons [54, Dicke quantum phase transition with a superfluid gas of atoms in an optical cavity [101, and BECs of photons in an optical microcavity [102. In fact, one can go way back and regard lasing as a non-equilibrium BEC phase transition. The nature of Bose-Einstein condensation in such non-equilibrium settings have attracted much interest, because of its intrinsic differences to the equilibrium condensation phenomena 103. Investigations of many-body effects in CRAs in such experimentally relevant driven-dissipative regime has only recently begun [52, 104, 105. The open nature of CRAs makes it a particularly interesting platform to study non-equilibrium many-body physics. The external driving here can easily be tuned to be incoherent, coherent, or quantum, opening the road for exploration of novel many-body phases out of equilibrium.

Before discussing the efforts in this area, we would like to mention that investigations on driven dissipative regimes in the context of semiconductor polaritons and cold atoms and ions systems have also been carried out, employing field-theoretical approaches [106, or more conventional quantum optical approaches [107. These have mostly focused on problems with specifically engineered baths [108] or (quasi-) exactly solvable spin and fermionic models or dynamical phenomena [109, 110.

In this section, we review the early and more recent works on non-equilibrium physics in dissipative $\mathrm{JCH}$ or $\mathrm{BH}$ models, with or without driving. While there has been a proposal to implement the attractive $\mathrm{BH}$ model directly in a circuit-QED system [111, the majority of experimental realizations of a CRA are of the JCH type where the two-level system could be a real or artificial atom. Therefore, we begin by looking at similarities and differences between the two models in a nonequilibrium setting, which was first investigated by Grujic and coworkers [112.

After setting the stage, we look at fermionization and crystallization of photons in driven dissipative settings. We then move on to dynamical (transient) effects, first looking at the dynamical signatures of superfluidinsulator transition and then at the so-called localizationdelocalization transition of photons due to self-trapping effect. The experimental verification of the latter in a circuit-QED platform is also reviewed. Then comes proposals to implement exotic out-of-equilibrium phases, viz photon supersolid and Majorana-ilke modes. The next section gives a brief survey of further interesting works that investigate photon correlations and entanglement in small-size lattices or using mean field as well as those that investigate phases in 2D lattices with or without artificial gauge fields. Finally, we close the section with a brief discussion on possible experimental platforms.

\section{A. Comparison between $\mathrm{JCH}$ and $\mathrm{BH}$ models}

Let us begin by describing how to model the drivendissipative systems. Firstly, coherent driving by laser fields can be modelled by introducing a driving term in the Hamiltonian, $H_{\text {drive }}=\sum_{i}\left[\Omega_{i}(t) a_{i}^{\dagger}+\Omega_{i}^{*}(t) a_{i}\right]$, where $\Omega_{i}(t)=\Omega_{i} \exp \left(-i \omega_{L} t\right)$ is the time-dependent driving field and $a_{i}$ is the driven resonator mode. As we have noted earlier, we will encounter two types of model Hamiltonians. One is the Bose-Hubbard Hamiltonian which, including the driving term, can be written as

$$
\begin{aligned}
H_{B H} & =\sum_{i}\left[\omega_{c} a_{i}^{\dagger} a_{i}+\frac{U}{2} a_{i}^{\dagger} a_{i}^{\dagger} a_{i} a_{i}\right]-\sum_{\langle i, j\rangle} J a_{i}^{\dagger} a_{j} \\
& +\sum_{i}\left[\Omega_{i}(t) a_{i}^{\dagger}+\Omega_{i}^{*}(t) a_{i}\right]
\end{aligned}
$$

while the other is the JCH Hamiltonian

$$
\begin{aligned}
H_{J C H} & =\sum_{i}\left[\omega_{c} a_{i}^{\dagger} a_{i}+\left(\omega_{c}-\Delta\right) \sigma_{i}^{+} \sigma_{i}^{-}\right. \\
& \left.+g\left(a_{i}^{\dagger} \sigma_{i}^{-}+a_{i} \sigma_{i}^{+}\right)\right] \\
& -\sum_{\langle i, j\rangle} J a_{i}^{\dagger} a_{j}+\sum_{i}\left[\Omega_{i}(t) a_{i}^{\dagger}+\Omega_{i}^{*}(t) a_{i}\right] .
\end{aligned}
$$

In both models, $\omega_{c}$ is the bare resonator frequency and $J$ is the photon hopping rate between the nearest neighbour resonators-denoted by the angled brackets. $\Delta$ is 
the detuning between the resonator and atom and $g$ is the atom-photon coupling strength. $\sigma_{i}^{+}$and $\sigma_{i}^{-}$are the atomic raising and lowering operators at site $i$.

Secondly, photon losses are modelled by assuming that the system is (weakly) interacting with a bath of harmonic oscillators. Taking the Born-Markov approximation, one obtains a quantum master equation describing the dynamics of the reduced density matrix of the system $\rho$ 113. In this review, we will mostly deal with master equations of the form

$$
\begin{aligned}
\dot{\rho}= & -i\left[H_{X}, \rho\right]+\frac{\gamma}{2} \sum_{i=1}^{N}\left(2 a_{i} \rho a_{i}^{\dagger}-a_{i}^{\dagger} a_{i} \rho-\rho a_{i}^{\dagger} a_{i}\right) \\
& +\frac{\kappa}{2} \sum_{i=1}^{N}\left(2 \sigma_{i}^{-} \rho \sigma_{i}^{\dagger}-\sigma_{i}^{\dagger} \sigma_{i} \rho-\rho \sigma_{i}^{\dagger} \sigma_{i}\right)
\end{aligned}
$$

where $X$ can be $J C H$ or $B H ; \gamma$ and $\kappa$ are the dissipation rates of photons and atoms, respectively, assumed here to be identical for all sites.

Now we are ready to compare the two models. Let us start from the simplest case: a single-site system. The eigenstates of the Jaynes-Cummings Hamiltonian are the 'dressed' states $|n, \pm\rangle$, where $n$ is the total excitation number and \pm labels two polaritonic (hybrid atom-photon) modes with energies $\omega_{n}^{ \pm}=n \omega_{c}-\Delta / 2 \pm$ $\sqrt{(\Delta / 2)^{2}+n g^{2}}$. Because of the dependence on $n$, the energy of $|2,-\rangle$ is not twice that of $|1,-\rangle$, as illustrated in figure 8 (a) for $\Delta=0$. Comparing with the energy levels of the Kerr-nonlinear model shown in figure 8 (b), one thus concludes that if '-' polaritons are driven and if the driving is sufficiently weak such that only the lowest states are occupied, an effective interaction strength $U_{\text {eff }}$ can be defined to formally connect the two models. That is, one can assign an effective $\mathrm{BH}$ parameter

$$
\frac{U_{e f f}}{g}=\frac{\omega_{2}^{-}-2 \omega_{1}^{-}}{g}=\frac{\Delta}{2 g}+2 \sqrt{\left(\frac{\Delta}{2 g}\right)^{2}+1}-\sqrt{\left(\frac{\Delta}{2 g}\right)^{2}+2},
$$

to the JCH model.
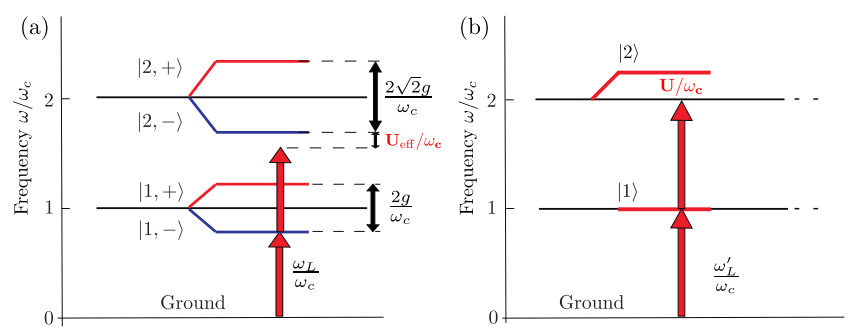

FIG. 8: Comparison between the lowest eigenmodes of (a) the Jaynes-Cummings model for $\Delta=0$ and (b) the Kerrnonlinear model. Reprinted from Grujic et al. [112].

Deep in the strongly interacting regime, we would thus expect the two models to yield similar qualitative behaviour: the polaritons will delocalize to minimize the kinetic energy, but the double occupation of any site is inhibited at the same time. On the other hand, we also know that the two models should converge in the opposite limit of linear CRAs. These two observations give a heuristic argument as to why we can see the Mottsuperfluid transition in a JCH system. However, this does not mean that the two types of systems are similar in all aspects. After all, there are additional atomic degrees of freedom in the JCH systems. To illustrate similarities and differences in the two models, figure 9 plots various expectation values of the non-equilibrium steady state (NESS) for a homogeneously-driven periodic threesite CRA.
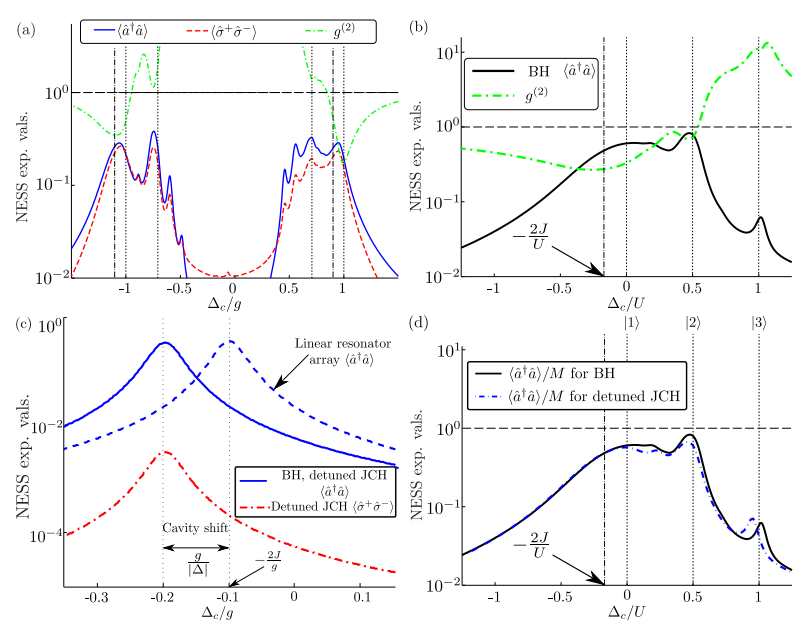

FIG. 9: Steady state expectation values for a homogeneously driven, cyclic three-site coupled resonator array. (a) A JCHtype system with $\Delta=2 J=2$ and (b) a BH-type system with the effective nonlinearity set by $g=20$. (c) $\Omega=0.3, g=20$, $\Delta / g=-10$ (d) $g=1.6 \times 10^{4} . \Omega=2$ in all figures except (c) and all parameters are in units of $\gamma$. Reprinted from Grujic et al. 112 .

Upon comparing the $\mathrm{JCH}$ (figure 9(a)) and the $\mathrm{BH}$ (figure $9(\mathrm{~b})$ ) results, we immediately see that the $\mathrm{JCH}$ has a more complex structure. Broadly, the spectrum is divided into two parts. The left (right) wing is mostly composed of the '-' (' + ') polaritons for the small value of $J$ used here $(U=20 J)$. The vertical dash-dotted line indicates the driven single-excitation eigenstate while the dotted lines indicate the eigenstates of a single resonator $(J=0$ case). A BH system by comparison has a relatively simple spectrum with peaks near (but not exactly at) the single-site $n$-photon energies indicated by the dotted vertical lines. Note that the spectrum is quite broad as we have assumed $\gamma=J$. Despite these differences, there is a notable similarity when the systems are driven at the single-particle resonance (vertical dash-dotted lines)-enhanced steady-state photon number is accompanied by a dip in the same-time intensityintensity correlation function $g^{(2)}=g^{(2)}\left(i_{1}, i_{1}\right)$. The lat- 
ter is defined as

$$
g^{(2)}\left(i_{1}, i_{2}\right)=\left\langle a_{i_{1}}^{\dagger} a_{i_{2}}^{\dagger} a_{i_{2}} a_{i_{1}}\right\rangle /\left\langle a_{i_{1}}^{\dagger} a_{i_{1}}\right\rangle\left\langle a_{i_{2}}^{\dagger} a_{i_{2}}\right\rangle,
$$

where the expectation values are taken with respect to the steady state density operator. The dip in $g^{(2)}$ signifies that two excitations do not want to occupy the same site simultaneously. This quantifies the photon blockade effect mentioned earlier, arising due to the strong effective repulsion between photons.

At this point, the reader might be wondering whether it is possible at all to achieve a good quantitative agreement between the two models. It is indeed possible in the 'photonic' regime, in which the atom-photon detuning is so large that the polaritons are mostly photonic. In this limit, however, the effect of atom-photon interaction is to merely shift the resonance (figure 9(c)). To counteract the reduced nonlinear effect, one needs a very large value of $g$. In figure $9(\mathrm{~d}), \Delta / g=-10$ and $g=6 \times 10^{4} \gamma$ were used. Since these kind of numbers lie well outside the experimental realm, we conclude that differences between the two types of systems are there in realistic systems and one must be careful in choosing the correct model to describe a given CRA. In particular, one should check whether a phenomenon found in a $\mathrm{BH}$ system can also be observed in a $\mathrm{JCH}$ system in a reasonable parameter regime. We will come across such examples below.

\section{B. Fermionization and crystallization of photons and polaritons in $\mathrm{BH}$ and $\mathrm{JCH}$ models}

The first investigations on many-body physics in driven-dissipative CRAs employed 1-dimensional $\mathrm{BH}$ models. Signatures of strong on-site interaction leading to fermionization of the excitations (much as in TonksGirardeau gases) were shown to be visible in the NESS of driven-dissipative CRAs systems 114, 115. JCH versions of these phenomena have also been studied by Grujic et al. 112-with large enough atom-photon coupling, the predicted fermionization in the $\mathrm{BH}$ system was shown to occur in driven-dissipative JCH systems.

\section{Fermionization of photons}

We begin with the work of Carusotto and coworkers [114]. The system is composed of Kerr nonlinear cavities coupled in a cyclic fashion as illustrated in figure 10. The master equation describing the system is given by (17) with $X=B H$, with homogeneous driving fields such that $\Omega_{i}=\Omega$. As shown on the right panel of figure 10 , the 'transmission' spectrum of the total 'transmitted' intensity $n_{T}=\sum_{i} \operatorname{Tr}\left[\rho a_{i}^{\dagger} a_{i}\right] \equiv\left\langle a_{i}^{\dagger} a_{i}\right\rangle$ show the traces of strong interaction between photons. For the case of impenetrable bosons $(U \gg J)$, the Bose-Fermi mapping can be employed [116], which translates an interacting Boson model into a free Fermion model to enable calculations of the energy eigenvalues. These eigenvalues with their eigenstates are shown in the figure, showing excellent agreement between the spectra of the drivendissipative system and the eigenvalues of the closed system.
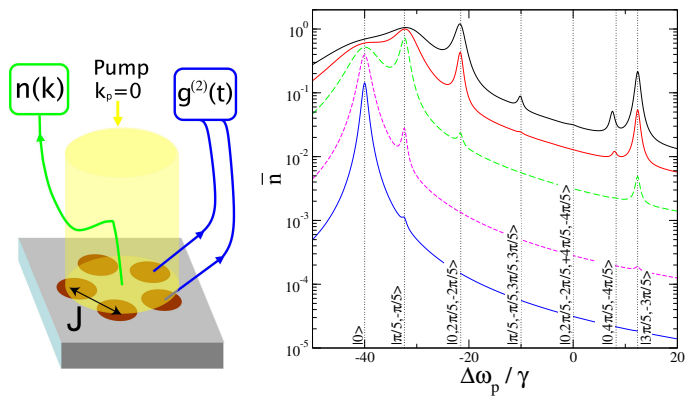

FIG. 10: Left: Schematic diagram of the system. Right: Total transmission spectra as a function of driving frequency for 5 cavities in the impenetrable boson limit, $U / J=\infty$ and $J / \gamma=20$. Decreasing pump amplitude from top to bottom: $\Omega / \gamma=3,2,1,0.3,0.1$. The vertical dotted lines indicate the predictions of the Bose-Fermi mapping where the states corresponding to the spectral peaks are written in the momentum basis. Reprint with permission from Carusotto et al. [114.

To further illustrate the fermionization of photons, figure 11 plots the on-site $\left(g^{(2)}\left(i_{1}=i_{2}\right)\right)$ and nearestneighbour $\left(g^{(2)}\left(i_{1} \neq i_{2}\right)\right)$ correlations as functions of $U / J$, when the pump lasers are kept on resonance with the lowest two-photon transition. Clearly, both the JCH and $\mathrm{BH}$ systems yield qualitatively similar results when the effective nonlinearity is matched via equation (18). In the weak interaction limit, all the levels are harmonic and the emitted light inherits the Poissonian nature of the pump. In the intermediate regime, $U / J \approx 1$, the (driven) two photon peak starts to separate from the single photon peak leading to bunching. Finally, in the large $\mathrm{U} / \mathrm{J}$ limit, the two photons fermionize, resulting in strong antibunching with enhanced cross-correlations.

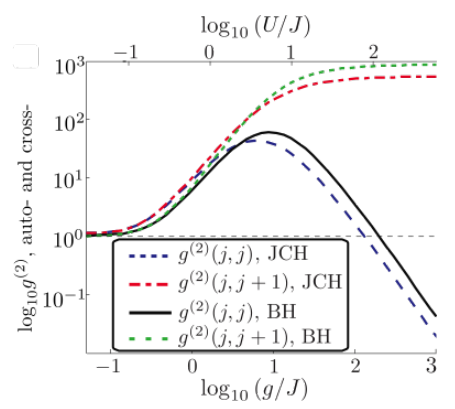

FIG. 11: Auto- (black, solid line) and cross- (red, dashed line) correlations as functions of the interaction strength. JCH systems with different couplings $g$ are compared with $\mathrm{BH}$ systems with $U$ calculated from (18). $J / \gamma=20, \Omega / \gamma=0.5$, and $\Delta=2 J$. Reprinted from Grujic et al. [112].

A detailed investigation on the fluorescence spectrum 
and the spectrum of the second-order correlation function under coherent and incoherent driving for a two coupled cavities of JCH type was carried out by Knap et al. [117. Similar to the work described above, the resonance peaks of the measured spectra of the dissipative system were matched to the eigenenergies of the closed system.

\section{Crystallization of polaritons}

In an independent work, Hartmann has studied the effects of strong interaction between polaritons in coupled resonators (described by the $\mathrm{BH}$ model in which $a_{j}$ is the polariton annihilation operator) [115. In that setup, instead of driving the system homogeneously, a flow of polaritons is created via modulation of the phase of the driving laser such that $\Omega_{j}=\Omega e^{-i \phi_{j}}$, where $\phi_{j}=j \pi / 2$. The interplay between this flow and the nonlinearity then results in polariton crystallization, where excitations are predominantly found within a specific distance from one another. To describe this phenomenon in detail, we will use the spatial correlation function $g^{(2)}(i, j)$ as well the momentum correlation function defined by

$$
g_{\mathrm{BM}}^{(2)}(p, q)=\left\langle B_{p}^{\dagger} B_{q}^{\dagger} B_{q} B_{p}\right\rangle /\left\langle B_{p}^{\dagger} B_{p}\right\rangle\left\langle B_{q}^{\dagger} B_{q}\right\rangle,
$$

where the momentum eigenmode operator is defined as $B_{p}=1 / \sqrt{N} \sum_{j} \exp (i p j) a_{j}$ with $N$ the total number of cavities.

There are two interesting regimes determined by the relative strength between the nonlinearity $U$ and the driving strength $\Omega$ : 1) Weakly nonlinear regime, $\Omega \gg U$, and 2) Strongly nonlinear regime, $U \gg \Omega$. Let us start with the weakly nonlinear regime. In this stronglydriven regime, a semiclassical treatment is valid, i.e., $B_{p}=\sqrt{N} \beta \delta_{p, \pi / 2}+b_{p}$, where $\beta$ is a mean amplitude for the driven mode at $p=\pi / 2$ and $b_{p}$ is an annihilation operator for the quantum of fluctuations. To second order in fluctuations the Hamiltonian, neglecting the driving term for now, can be written as

$$
\tilde{H}=\sum_{p}\left[\left(\omega_{p}+2 U n\right) b_{p}^{\dagger} b_{p}+\left(\frac{U \beta^{2}}{2} b_{p}^{\dagger} b_{\bar{p}}^{\dagger}+\text { H.c. }\right)\right],
$$

where $\bar{p}=\pi p /|p|-p$ and $n=|\beta|^{2}$ is the mean number of polaritons. This Hamiltonian is known to exhibit two-mode squeezing between the modes $p$ and $\bar{p}$, arising due to the scattering between $p$ and $\bar{p}$ modes induced by the nonlinear term. The latter also induces densitydensity correlations between the modes, which can be seen from the intensity correlation function between the Bloch modes evaluated to the leading order in $1 /(N n)$ :

$$
\begin{aligned}
g_{\mathrm{BM}}^{(2)}(p, q) & \approx 1+\delta_{p \neq \pi / 2}\left(\delta_{p, q}+\delta_{p+q, \pm \pi} \frac{|g|^{2}}{m^{2}}\right) \\
& -\delta_{p, \pi / 2} \delta_{q, \pi / 2} \frac{2 m}{N n}
\end{aligned}
$$

where $m=2 U^{2} n^{2} /\left(12 U^{2} n^{2}+\gamma^{2}\right)$ is the contribution to the mean photon number from quantum fluctuations and $g=\left\langle B_{p} B_{\bar{p}}\right\rangle=-\beta^{2}\left(4 U^{2} n+i U \gamma\right) /\left(12 U^{2} n^{2}+\gamma^{2}\right)$. The term $\delta_{p+q, \pm \pi} \frac{|g|^{2}}{m^{2}}$ clearly shows the correlations between the modes $p$ and $\bar{p}$ for non-driven modes, while the driven mode $p=\pi / 2$ exhibits slight antibunching $g_{m}^{(2)}(\pi / 2, \pi / 2) \approx 1-2 m / N n$.

In the strongly nonlinear regime, the semiclassical description is no longer valid. Instead, the time evolving block decimation (TEBD) algorithm (see [118, 119] for reviews) was used to calculate the steady state for an array of 16 cavities (for other methods to deal with larger systems, see e.g., [120, 121]). Contrary to the weakly nonlinear regime, there is now strong correlations between the same Bloch modes $\left(g_{m}^{(2)}(p, p)>1\right)$, while different modes are strongly anticorrelated $\left(g_{m}^{(2)}(p, q)<1, p \neq q\right)$, as shown in figure 12 .
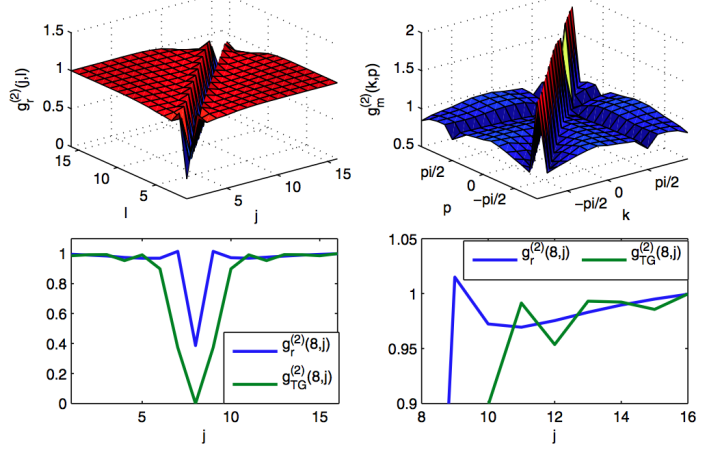

FIG. 12: Intensity correlations of the steady state for 16 cavities in the strong interaction regime driven at resonance. $U / \gamma=10, J / \gamma=2, \Omega / \gamma=2$ and $\Delta_{L}=0$, where $\Delta_{L}$ is the polariton-laser detuning. Top row: steady state intensity correlations between spatial modes (left) and Bloch modes (right). Bottom row: comparison with the correlation functions of a Tonks-Girardeau gas. Reprinted with permission from Hartmann [115.

The same figure also shows an evidence of photon crystallization, by which we mean the following: if a polariton is found in one resonator, a second polariton is most likely to be found in an adjacent resonator and this happens at the reduced probability of finding a polariton at farther sites. This is clearly observed in the polariton correlation function between the cavities, where $g^{(2)}(j, j+1)>1$ and $g^{(2)}(j, j+k)<1$ for $k=1,2, \ldots$. The correlations fall below 1 only when the driving phase is $\neq 0$, indicating that the observed phenomenon emerges due to the interplay between the nonlinearity and the flow of polaritons. For comparison, figure 12 also plots (green lines) the density correlations of a Tonks-Girardeau (TG) gas $g_{\mathrm{TG}}^{(2)}(i, j)=1-(\sin (\pi \tilde{n}(i-j)) / \pi \tilde{n}(i-j))^{2}$, where $\tilde{n}$ is the number of polaritons per resonator. While the TG gas shows an oscillation in the density-density correlation, no such oscillation is observed in crystallized polaritons. Similar behaviour is observed for the JCH model when the polaritonic mode $|1,-\rangle_{p=\pi / 2}$ is driven, as depicted 
in figure 13 . It is interesting to note that the on-site repulsion is strengthened with increasing $\Delta / g$-this can be attributed to an increase in the atomic portion of the JC polariton with the detuning.

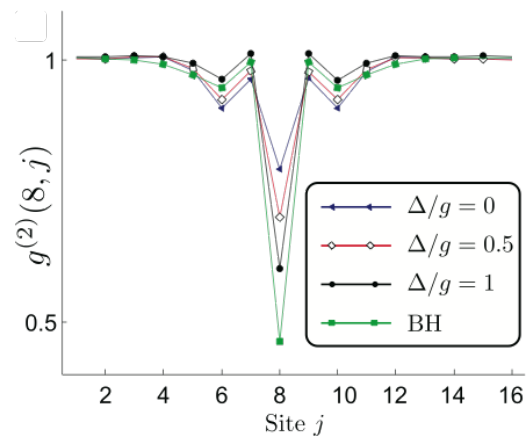

FIG. 13: Steady state photon density-density correlations for the JCH model for various values of $\Delta / g$ as compared with that from the BH model. $g / \gamma=10\left(U_{\text {eff }} \approx 6\right), J / \gamma=2$, and $\Omega=2$. Reprinted from Grujic et al. [112].

\section{Dynamical phenomena}

In the previous subsection we have seen that the steady states of driven-dissipative CRAs are capable of exhibiting signatures of underlying many-body states in the equilibrium system and furthermore that interesting phases can be created due to driving. However, this is not the only non-equilibrium scenario that reveals interesting physics. One well-known example is quenching, in which the system is initially excited in a particular initial state and subsequent dynamics is observed. Here we review two early works taking this approach. We begin with the non-equilibrium study on superfluid-insulator transition by Tomadin et al. 122 and briefly summarize a similar study on a hybrid system by Hummer et al. [123. Then we review the 'localization-delocalization' transition of photons 124, which has been verified experimentally in a superconducting circuit setup [125].

\section{Superfluid-insulator transition}

In [122, Tomadin and coworkers investigated a dissipative $\mathrm{BH}$ system in 2 dimensions without any driving field, initially prepared in a Mott-like state. Much as in the quantum quench scenario, subsequent transient evolutions depend sensitively on the underlying equilibrium phase (without dissipation). A self-consistent cluster mean-field approach was employed with up to two sites per cluster, where the evolution within a cluster is exact but the interaction with the rest of the system is treated at the mean-field level.

Let us first consider the dynamics of the initial Mott state. With negligible dissipation, the dynamics obvi- ously depends sensitively on the value of $J / U$. For small $J / U$, the system is expected to stay more or less in the same state, whereas for large $J / U$, nontrivial dynamics are expected. As in the equilibrium case, a useful observable to distinguish between these distinct cases is the second-order correlation function, since strong antibunching is expected to persist for small $J / U$. In fact, in the transient scenario, a more useful observable was shown to be the time-averaged second-order correlation function, which we denote $\langle g\rangle_{t}$. Figure 14 illustrates the usefulness of this quantity, where the averaging is over $2 / \gamma<t<4 / \gamma$. The inset shows the time-averaged meanfield order parameter $\langle|\psi|\rangle_{t}$ displaying similar behaviour as $\langle g\rangle_{t} ; z$ is the coordination number denoting the number of nearest neighbours. For small $J / U$, the average intensity correlation is 0 indicating that the system remains in the initial Mott state. However, for large $J / U$, the initial state becomes unstable and a crossover towards Poisson statistics $\left(g^{(2)}=1\right)$ is observed, the latter occurring over a narrow region of $J / U$ near the critical point indicated by the vertical dashed line.

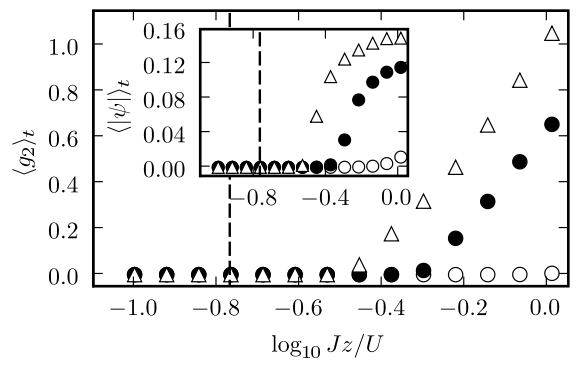

FIG. 14: Time averaged intensity correlations averaged in the interval $2 / \gamma<t<4 / \gamma^{-1}$. The laser field is resonant to the bare cavity frequency while the dissipation rate takes the values $\gamma / U=4 \times 10^{-2}$ (empty circles), $\gamma / U=2 \times 10^{-2}$ (filled circles), and $\gamma / U=1 \times 10^{-2}$ (empty triangles). The inset shows the mean-field order parameter. The vertical dashed line indicates the critical point where the Mott-superfluid phase transition occurs in the equilibrium case. Reprinted with permission from Tomadin et al. 122.

Experimentally, the initial state is most likely to be prepared by a series of laser pulses. In this case, there will inevitably be some imperfections in preparing the Mott state. Tomadin et al. have studied the effect of such imperfections by considering non-ideal filling factors in the initial state. Figure 15 shows the results. Clearly, a deviation from the ideal initial state does not destroy the stark difference between the superfluid (triangles) and Mott (circles) regimes as measured by $\left\langle g^{(2)}\right\rangle_{t}$. The same conclusion holds for a general initial condition, with up to $20 \%$ depletion of the average filling, for the whole range of initial coherences allowed by the manifold spanned by 0 and 1 Fock states in each resonator.

A similar investigation on non-equilibrium signatures of the phase transition has been carried out by Hummer et al. 123, in a hybrid system of flux qubits coupled 


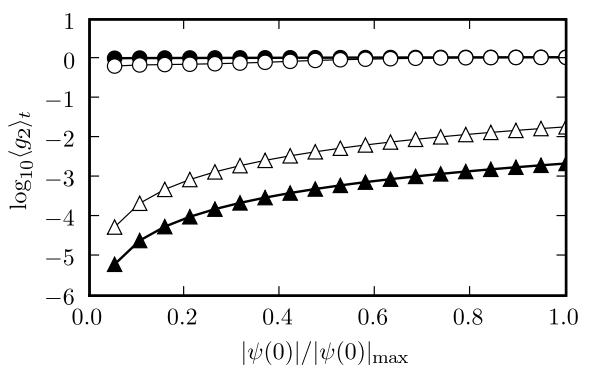

FIG. 15: Time-averaged intensity correlations, averaged over the interval $2 / \gamma<t<4 / \gamma^{-1}$, for resonant driving and $\gamma=2 \times$ $10^{-2}$. The vacuum population of the initial state is $\rho_{0}=0.02$ for the filled symbols and $\rho_{0}=0.2$ for the empty symbols. Results below the critical point $(z J / U=0.1)$ are denoted by triangles while those above the critical point $(z J / U=$ 1.0, ) are denoted by circles. Reprinted with permission from Tomadin et al. [122].

with NV centers. The model describing this system is different from the $\mathrm{BH}$ system in that i) each site comprises of qubits coupled to collective bosonic modes of NV centers surrounding it, and ii) the coupling is between the qubits, not bosons. Despite these differences, the time averaged probability to find two excitations in a single site is shown to be a very good indicator of the underlying phase transition in the equilibrium case.

\section{Localization-delocalization transition of photons}

The transient phenomenon to be reviewed here is not associated with the underlying phases of the equilibrium system, but with the dynamics of the system. The concept was initially introduced by Schmidt et al. 124] essentially in a closed Jaynes-Cummings dimer, but the subsequent experimental demonstration [125] showed that dissipation can play an important role in achieving the transition dynamically. As this experiment provides the state-of-the-art realization of a CRA, we discuss it in some detail. The essential physics of the localization-delocalization transition is similar to that of self-trapping: when two nonlinear systems are tunnelcoupled, the oscillation between the systems can be inhibited by the on-site nonlinearity. While previous studies in optical fibers [126, molecules [127, cold atoms [128130], and polaritons [131] have concentrated on the Kerr nonlinearity, Schmidt et al. showed that the same phenomenon also occurs in a Jaynes Cummings dimer. The effect has been generalized to more than two sites by Schetakis and coworkers [132.

As first explained in [124, the physics of the localization-delocalization transition are nicely captured in the semiclassical picture without dissipation. One resonator is assumed to be initially in a coherent state with $N$ photons on average, while the other resonator is assumed to be empty. Then, the semiclassical anal- ysis shows that the photon imbalance $Z(t)=\left(n_{L}(t)-\right.$ $\left.n_{R}(t)\right) / N$ undergoes an oscillation whose frequency decreases with increasing $g$ and furthermore goes to 0 at the critical coupling strength $g_{c}^{c l} \approx 2.8 \sqrt{N} J$. This behaviour survives in the quantum regime as shown in figure 16 for a lossless dimer with 7 photons initially placed in the left resonator. $\bar{Z}(t)$ is the time averaged imbalance up until time $t$. While the initial imbalance is washed out over time below the critical coupling strength, $g=0.3 g_{c}^{c l}$ (figure 16(a)), the signature of photon localization is evident above the critical coupling strength, $g=2.0 g_{c}^{c l}$ (figure 16(b)). The localization behaviour can be explained as follows. For a symmetric dimer, the two states with opposite imbalance 1 and -1 are approximate eigenstates of the system when $g \gg J$. Using the degenerate perturbation theory the period of oscillation between these states can be calculated as $1 /\left(c_{N_{0}} J(J / g)^{N_{0}-1}\right)$ with $c_{N_{0}}$ a constant that depends on the total photon number $N_{0}$. Clearly, this number diverges rapidly with increasing $N_{0}$ when $J / g \ll 1$.
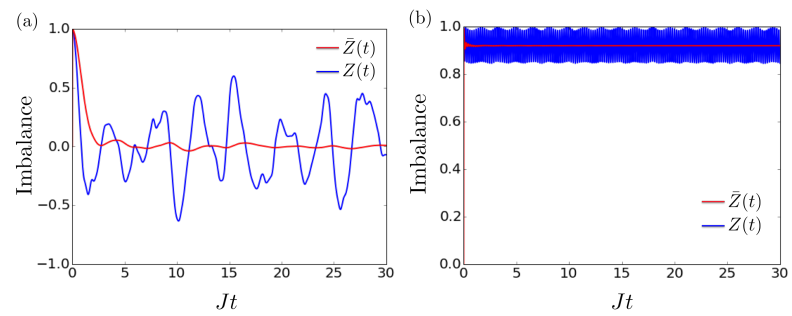

FIG. 16: Photon number imbalance (blue curves) and the time-averaged imbalance (red curves) for a lossless dimer containing 7 photons initially localized in the left resonator. (a) Below the critical coupling strength, $g=0.3 g_{c}^{c l}$, exhibiting delocalized dynamics. (b) Above the critical coupling strength, $g=2.0 g_{c}^{c l}$, exhibiting localized dynamics.

A detailed analysis shows that there are features not captured by the semiclassical treatment such as a beating in the coherent oscillation that is observable in the weakly-nonlinear case, smoothening of the transition, and a shift in the critical coupling strength where $g_{c}^{q u}<$ $g_{c}^{c l}$ 124. However, rather than delving into these features further, let us move on to the experimental realization of the dissipative localization-delocalization transition, where dissipation plays a key role in driving the transition dynamically. The latter occurs due to the photon number dependence of the critical coupling strength. For an experimental setup with fixed $g$, the initial photon number $N_{0}$ decides whether the system is in the localized regime $\left(g>g_{c}^{q u}\left(N_{0}\right)\right)$ or in the delocalized regime $\left(g<g_{c}^{q u}\left(N_{0}\right)\right)$. In a closed system, the system has no choice but to remain in a given regime. However, in a dissipative system, the decrease in the total number of photons can take the system from a delocalized regime to a localized regime (because $g_{c}^{q u}$ decreases with $N$ ).

This effect has been experimentally demonstrated by Raftery and coworkers in a superconducting circuit plat- 
form [125]. In the experiment, the initial state was prepared in a coherent state instead of a Fock state, but the described physics remain qualitatively unchanged. Initially, the qubits and the resonators are far-detuned so they are practically uncoupled. Then the system is driven by a coherent pulse and left to evolve a specific period of time, which prepares the system with the imbalance 1. The nonlinearity is then rapidly increased by bringing the qubits into resonance with the resonator mode. The results for various initial photon numbers $\left(N_{i}\right)$ are plotted in figure 17. It shows the homodyne signal in the initially unoccupied resonator as a function of the initial photon number and time. For a small enough $N_{i}$, the system starts off and remains in the localized regime, whereas for a larger $N_{i}$ the system starts off in the delocalized regime and crosses over to the localized regime after enough photons are lost from the system so that the critical coupling strength is smaller than the experimental coupling strength.

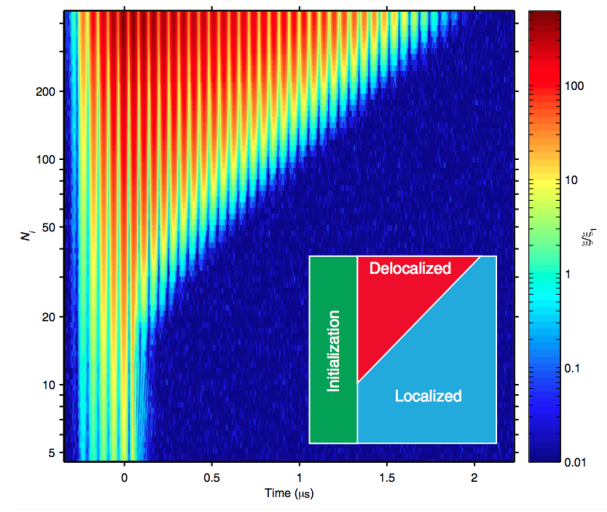

FIG. 17: Phase diagram of the dissipation-induced delocalization-localization transition for an open JaynesCummings dimer. Homodyne signals from the initially unoccupied resonator as a function of initial photon number $N_{i}$ and time. For $N_{i} \gtrsim 20$ the system starts in the delocalized regime because $g<g_{c}^{q u}(N)$. However, as time progresses the system enters the localized regime as photon losses drive the system into the localized regime characterized by $g \geq g_{c}^{q u}(N)$. Reprinted from Raftery et al. [125].

\section{Exotic phases out of equilibrium}

Coupled resonator arrays, especially in the superconducting circuit platform, allow one to engineer different types of interactions that are difficult to achieve in other systems. Here, we look at two proposals utilizing this advantage to realize exotic phases in non-equilibrium scenarios. We begin by reviewing a proposal on a photonic superfluid phase in a driven-dissipative setup and then a proposal to achieve Majorana-like modes in a chain of driven cavities.

\section{Photon supersolid phase}

Let us start with the proposal by Jin and coworkers, which investigated the consequences of adding a crossKerr term between nearest neighbours [133, 134. Such a term naturally arises in electronic systems due to the Coulomb interaction between electrons, but are necessarily much smaller than the on-site interaction term. However, in CRAs the magnitude of the new term can be made arbitrarily large as compared to the on-site interaction term, giving rise to interesting new phases. The open extended Hubbard system studied by the authors is described by the master equation (17) with

$$
H_{X}=H_{B H}+V \sum_{\langle i, j\rangle} n_{i} n_{j},
$$

which, as they show, can be realized using nonlinear coupling elements between the cavities.

Consider a 2D bipartite lattice-lattice that divides into two sublattices-with homogeneous coherent pumping $\left(\Omega_{i}=\Omega\right)$. Due to the presence of the cross-site interaction, a non-zero photon number difference between the sublattices may develop. This broken symmetry phase is characterized by the order parameter $\Delta n=\left|\left\langle n_{A}\right\rangle-\left\langle n_{B}\right\rangle\right|$, where $A$ and $B$ are sublattice indices. Figure 18(a) shows the order parameter as a function of the hopping strength and cross-Kerr nonlinearity for $U=1$. The coloured region indicates the broken-symmetry (or crystalline) phase, where the photon number distribution exhibits a checkerboard pattern. We see that the interaction term favours the broken symmetry phase, whereas the hopping tends to restore the symmetry. Remarkably though, having $V \neq 0$ is not a prerequisite to obtain the checkerboard phase, unlike in the equilibrium counterpart [133]: it is possible, although much harder, for the non-equilibrium steady-state to have non-zero $\Delta n \neq 0$ even with $V=0$. The requirements are a non-zero initial imbalance and a finite hopping strength.

In figure $18(\mathrm{~b})$, the effects of driving field detuning, $\delta$, is shown for $U=0$ and $z V=0.6$. Here the situation gets more interesting. A new phase appears (shaded green area) where the steady-state never becomes truly stationary in that there is a residual time dependence of $\langle a\rangle$ (on top of a trivial time dependence due to driving). A closer inspection on the reduced density matrix of the sublattice shows that there is a global dynamical phase coherence on top of the checkerboard pattern, which led Jin and coworkers to call this phase a photonic supersolid phase. Similar phases were also found in the equilibriums setting for an extended JCH model with the nearest neighbour interaction between the qubits 135.

The existence of the crystalline phase was corroborated by performing numerical simulations using the matrix product operator technique in 1D [115]. This approach takes into account quantum fluctuations ignored in the mean-field analysis and is therefore much more reliable. The results are shown in figure 19. The nature of the 

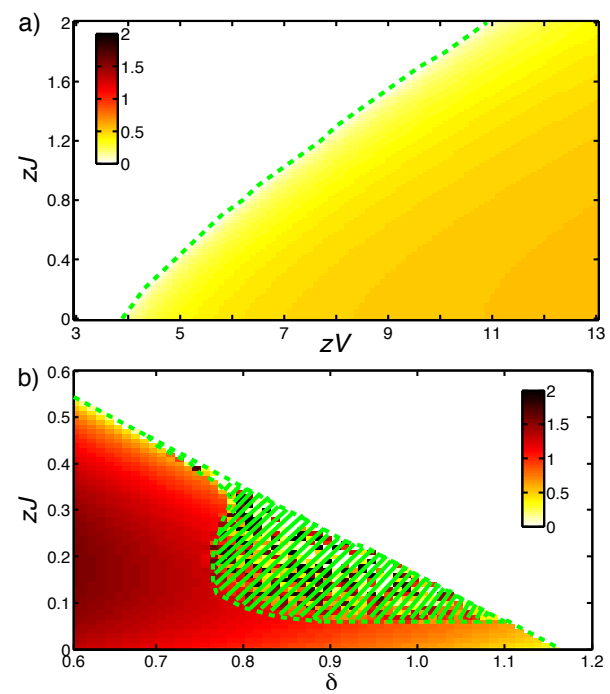

FIG. 18: a) Order parameter as a function of $J$ and $V$, for $\delta=$ 0 and $U=1$. b) Order parameter as a function of $J$ and $\delta$, for $z V=0.6$ and $U=0 . \Omega=0.75$ for both diagrams. For finite values of the detuning (of the driving field), an intermediate regime between staggered and uniform phase appears, which can be seen as a nonequilibrium analogue of supersolid. Green dashed lines are there to provide a guide to the eye. Reprinted with permission from Jin et al. 133.

crystalline phase is displayed in the second-order intensity correlation function $g^{(2)}(i, j)$. Figure 19 (a) plots this function for 20 cavities in the absence of hopping $(J=0)$. In this regime, the staggered distribution of photons is manifest, especially for larger values of $V$. Figure 19 (b) plots $g^{(2)}$ for 21 cavities for various values of hopping strengths, displaying the suppression of crystalline phase with increasing hopping strength.

\section{Majorana-like modes}

As the last detailed review of this section we look at a proposal by Bardyn and Imamoglu on a photonic realization of Majorana-like modes (MLMs) in a 1D CRA 136. The non-equilibrium steady state of the drivendissipative version of the model has been studied by Joshi and coworkers in a different context [137. There the model was shown to be equivalent to a transverse field anisotropic XY model, whose NESS was calculated using a matrix product operator representation. Quantum entanglement and correlations near the critical point (of the equilibrium system) were found to reveal interesting similarities and differences to the equilibrium physics. For instance, singular behaviour was found in the isotropic limit where the range of entanglement diverges (with vanishing magnitude), which is very distinct from the ground state behaviour. We refer the interested reader to the original article.

The Majorana modes are exotic 'half-a-fermion' zero
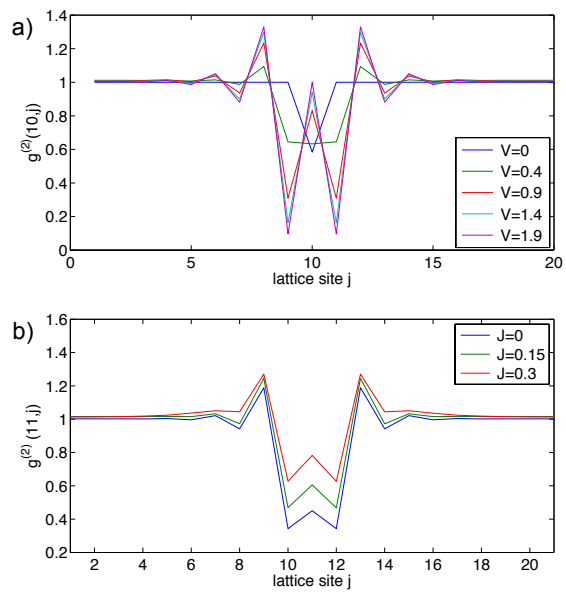

FIG. 19: Matrix product operator results for 1D CRA. (a) $g^{(2)}(10, j)$ for $\delta=0, J=0, U=0.5$, and $\Omega=0.4$. (b) $g^{(2)}(11, j)$ for $\delta=0, V=1, U=1$, and $\Omega=0.4$. Reprinted with permission from Jin et al. [133.

energy modes that can form, among others, in a 1D topological p-wave superconductor described by Kitaev's toy model [138]. The latter involves the so-called p-wave paring term, $c_{i} c_{i+1}+$ h.c., where $c_{i}$ is the fermion annihilation operator at site $i$, and is crucial for the formation of Majorana modes. To realize Kitaev's model with photons, the authors employ the hard-core limit $(U>>J)$ of the $\mathrm{BH}$ model and a parametric driving of the intercavity field as shown in figure 20. In the hard-core limit $(U>>|\Delta|)$, the effect of the parametric driving (with an amplitude $\left.\Delta=|\Delta| e^{i \phi}\right)$ is to introduce the 'squeezing' term

$$
H_{\text {drive }}=|\Delta| \sum_{i}^{N-1}\left(e^{i\left(2 \omega_{p} t+\phi\right)} \tilde{a}_{i} \tilde{a}_{i+1}+\text { H.c. }\right),
$$

where $\tilde{a}_{i} \mathrm{~s}$ are the hard-core photon operators. Using the Jordan-Wigner mapping $c_{i}=\prod_{j=1}^{i-1}\left(-\sigma_{j}^{z}\right) \sigma_{i}^{-}$with $\sigma_{i}^{-}=$ $\tilde{a}_{i}$, the total Hamiltonian is written as

$$
\begin{aligned}
H= & -J \sum_{i=1}^{N-1}\left(c_{i}^{\dagger} c_{i+1}+\text { H.c. }\right)+|\Delta| \sum_{i=1}^{N-1}\left(e^{i \phi} c_{i} c_{i+1}+\text { H.c. }\right) \\
& -\mu \sum_{i=1}^{N-1} c_{i}^{\dagger} c_{i},
\end{aligned}
$$

where $c_{i}$ 's are fermionic operators and $\mu=\omega_{p}-\omega_{c}$ is the pump-resonator detuning.

The above Hamiltonian is identical to Kitaev's model of 1D p-wave superconductor of spinless fermions and hosts localized zero-energy Majorana modes [138. The latter can most readily be seen in the limit $J=\Delta>0$ and $\mu=0$, in which the Hamiltonian can be written as

$$
H=i J \sum_{i=1}^{N-1} d_{2 i}^{\dagger} d_{2 i+1}=-J \sum_{i=1}^{N-1} \sigma_{i}^{x} \sigma_{i+1}^{x},
$$




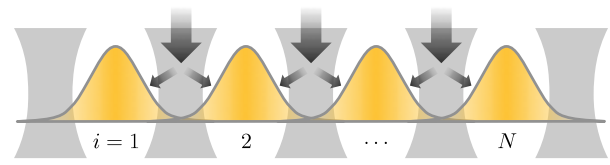

FIG. 20: Schematic diagram of a cavity array setup for realizing Kitaev's toy model. Cavity modes have strong Kerrnonlinearity to inhibit occupation of multiple photons, while the parametric pumping of the inter-cavity fields produce the 'p-wave' term. Reprinted with permission from Bardyn and Imamoglu [136.

where the Majorana operators are defined as $d_{2 i-1}=c_{i}+$ $c_{i}^{\dagger}$ and $d_{2 i}=-i\left(c_{i}-c_{i}^{\dagger}\right)$. The Majorana operators $d_{1}$ and $d_{2 N}$ are absent from the Hamiltonian and therefore the energy does not depend on whether or not the non-local fermion $f=\left(d_{1}+d_{2 N}\right) / 2$ is present. This means that the ground state is two-fold degenerate, characterized by the Majorana qubit operator

$$
\sigma_{M}^{z}=i d_{1} d_{2 N}=\prod_{j=1}^{N}\left(-\sigma_{j}^{z}\right) \sigma_{1}^{x} \sigma_{N}^{x} .
$$

The stringlike operator $P \equiv \prod_{j=1}^{N}\left(-\sigma_{j}^{z}\right)$ corresponds to the parity operator of the total number of (hardcore) photons. It commutes with the Hamiltonian and is therefore conserved, meaning that the Majorana qubit is associated with the end qubits only, thus (topologically) protected from local perturbations that preserves parity.

In the proposed photonic system, local dissipation breaks the parity and therefore also the topological protection of the Majorana qubit. However, the existence of localized Majorana modes and their characteristic nonAbelian braiding do not depend on the parity protection, and can therefore be simulated. The Majorana modes can be detected by attaching two probe cavities at the two ends of the array and measuring their second order cross-correlations. Due to the so-called Majorana mediated Cooper pair splitting [139, 140, the nonlocal Majorana qubit mediates a nonlocal coherent exchange of photons between the probe cavities, and the cross-correlation function reveals strong bunching in the topologically nontrivial regime as shown in figure 21 .

The braiding operation can also be simulated by using the 'tunnel-braid' operation [141, where the Majorana edge mode in one end of the array is tunnel-coupled, via an intermediate cavity, to the Majorana edge mode in another array. A sequence of operations such as turning on and off the tunneling amplitude to the intermediate cavity can be performed to simulate the braiding operation.

\section{E. Further interesting works}

There are many more works that address interesting out-of-equilibrium physics arising in CRAs, which we did

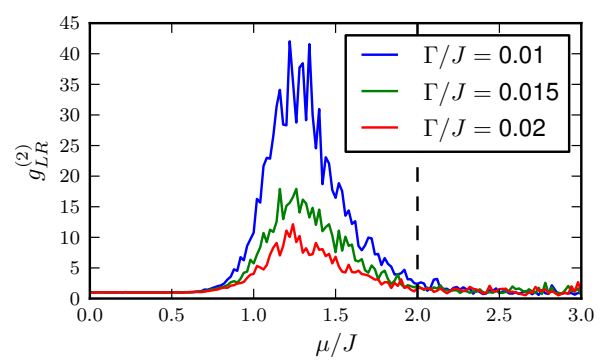

FIG. 21: Second-order cross-correlation function between the left and right probe cavities as a function of the detuning $\mu$ for 10 cavities (excluding probe cavities), $\Delta / J=1$, and $J_{L, R} / J=0.02$. $\Gamma / J=\Gamma_{L, R} / J$ denote cavity loss rates. Majorana-like modes are expected in topologically nontrivial regime (left of the vertical dashed line). Strong bunching is observed inside this region for large $\mu / J$ and for small dissipation rates. Reprinted with permission from Bardyn and Imamoglu [136].

not have the space to cover in detail. Here, we provide a brief survey of these works for the interested reader.

Photon statistics in driven-dissipative $\mathrm{BH}$ or $\mathrm{JCH}$ dimers have been investigated by many authors. One of the main themes is how the photon statistics, as measured by the local intensity-intensity correlation function $g^{(2)}$, change with the nonlinearity-hopping ratio. Gerace and coworkers showed that photon correlations can be used to reveal strongly correlated physics in a photonic Josephson junction-a three-site array with two end cavities coherently driven and the middle site containing single-photon nonlinearity [142]. Ferretti et al. have studied a homogeneously driven case for a $\mathrm{BH}$ dimer and found a crossover between coherent and anti-bunched regimes [143. An independent study on the JCH dimer was carried out by Leib and Hartmann, by mapping the system to an attractive BH model 144. Driving one of the cavities and measuring $g^{(2)}$ on the other, they observed the same crossover. A more detailed study on photon 'transport' was carried out by Biella et al. for up to 60 Kerr-nonlinear resonators, in which both the transmission spectrum and second-order correlation functions were evaluated 145. Going beyond coherent driving, effects of quantized driving fields were investigated by sending two-photon input states into a BH dimer [146. Effects of incoherent driving was also investigated by Ruiz-Rivas and coworkers, in which a spontaneous buildup of collective coherence was found [147].

The crossover from the weak to strong hopping for a dimer as well as the infinite lattice was investigated by Nissen and coworkers [148. There, in the dimer case, the antibunching was shown to persist up to a large value of hopping $(\mathrm{J} / \mathrm{g} \approx 10)$, for driving strengths comparable to or larger than the dissipation rate. However, a semiclassical analysis in the limit of large coordination number shows that the antibunching disappears with increasing system size. On the other hand, photon super- 
bunching was also shown occur in driven dissipative coupled resonator arrays, if the laser driving is tuned to the lowest-lying two-photon (polaritonic) mode in the $\mathrm{BH}$ (and JCH) system as shown by Grujic et al. [149. In fact, we have already observed the same behaviour while discussing fermionization of photons. TEBD simulations of 2-7 coupled JC cavities with homogeneous driving shows that when the hopping strength $J$ is larger than a critical value, the system exhibits photon (super) bunching, the magnitude of which decreases with increasing system size. Lastly, we want to discuss an interesting effect called unconventional photon blockade. Normally, a large value of nonlinearity $(U, g>>\gamma)$ is necessary to induce photon antibunching. However, Liew and Savona discovered that antibunching can occur in a weakly nonlinear $(U<\gamma)$ dimer upon making a judicious choice of parameters [150]. Soon after, the origin of this effect was identified as a destructive quantum interference between excitation pathways [151] and a similar effect has been shown to arise in resonators with a $\chi^{2}$ nonlinearity [152].

Quantum entanglement in driven-dissipative CRAs has also been investigated. Angelakis et al. have studied entanglement between polaritons formed at two cavities, where the latter are coupled via an empty resonator mode 153. Furthermore, a generalization to three sites that are interconnected via three coherently-driven waveguides modes allows the entanglement to be controlled [154, 155. In an alternative scheme, Liew and Savona considered three-site $\mathrm{BH}$ system with the middle site driven and found steady-state entanglement between the spatially separated modes [156]. An interesting aspect of this work is that, just like in the authors' antibunching work, only a small amount of nonlinearity is required. Furthermore, by repeating the three site setup, a multimode entanglement can be generated [157].

Finally, we would like to mention studies in 2D systems. We have already come across the latter when we reviewed non-equilibrium signatures of superfluidinsulator transition 122 and the photonic supersolid phase [133, 134. The phase diagram of the drivendissipative $\mathrm{BH}$ array (without the extra cross-Kerr interaction term) was studied by Le Boité and coworkers [158, 159. While the Mott-lobe-like structures were found, the phase diagram in the non-equilibrium setting exhibits clear differences to its equilibrium counterpart. This points to rich many-body physics beyond the equilibrium physics that arises due to the interplay between driving and dissipation. Verification of these mean-field results is an important future direction as predicted effects such as bistability may not survive the quantum fluctuations (see, for example [160] where tensor networks simulations for $1 \mathrm{D}$ arrays up to 60 sites have been performed). While one dimensional arrays can be numerically simulated efficiently, an efficient method for $2 \mathrm{D}$ arrays is still missing. Towards this direction, new numerical methods such as the 'corner-space renormalization method' 161] and 'self-consistent projection operator theory' [121] are currently being developed.
An interesting possibility in $2 \mathrm{D}$ that is absent in $1 \mathrm{D}$ is to introduce artificial magnetic fields by inducing phasedependent hopping terms. This allows one to implement quantum Hall-like models for photons as we have looked at earlier. Driven-dissipative signatures in such systems were studied by various authors. Umucallar and Carusotto proposed a method to experimentally verify the fractional quantum Hall state hidden in the NESS of a weakly driven array 96. In a similar setting, Hafezi and coworkers showed how $g^{(2)}$ measurements can be used to infer the underlying fractional quantum Hall states [98. Efforts to devise methods to experimentally measure topological invariants in integer quantum Hall systems have also been made. Ozawa and Carusotto showed that the Chern number and the Berry curvature can be measured by following the displacement of a localized excitation by a coherent driving field [162, while Berceanu et al. showed that by adding a weak harmonic potential to the model, the 'momentum-space Landau levels' can be observed [163. Alternatively, Hafezi 164] and Bardyn et al. 165. showed that twisted boundary conditions can be implemented to experimentally measure the topological invariants. Methods to stabilize strongly-correlated states of light using auxiliary two-level atoms have also been suggested [166, 167].

\section{F. Experimental implementations}

Before we finish this section, perhaps a few words on experimental realizations are in order. There are a number of promising experimental platforms to realize the JCH (or the BH) model: superconducting circuits, photonic crystal devices with quantum dots, microcavities connected by optical waveguides, polaritons in microstructures, and trapped ions. The trapped ion implementation uses phonons, the quantized vibrational motions of trapped ions, instead of photons and have been experimentally demonstrated for the dimer case [168]. 2D patterns of micropillars etched out of a semiconductor microcavity that can host exciton polaritons is a viable experimental platform especially if the polaritonic nonlinearity can be enhanced to the few-photon level. Recently, spin-orbit coupling has been demonstrated in this platform [169]. Microcavities plus optical waveguides is an innovative platform suggested by Lepert et al. 48] that uses cavities formed by open microcavities closed by coated waveguide tips. An emitter placed in each resonator acts as a two-level atom, while evanescent coupling between waveguides provide the photon hopping mechanism. Photonic crystals are dielectric devices with periodically patterned holes, where missing holes called defects can act as cavities. When a semiconductor is used to make a crystal, a quantum dot (artificial atom) grown in each defect resonator can allow the resulting system to be described by the JCH model. Majumdar et al. have experimentally implemented two cavities coupled to a single quantum dot in this system [170. The 
platform that is most promising is superconducting circuits. The JC dimer has already been implemented by Raftery et al. 125] as we have already seen (the $\mathrm{BH}$ one has also been implemented by Eichler et al. [171), and building a larger lattice seems certainly within reach in the near future with lattices up to 200 resonators in a Kagome geometry already a reality (without atoms yet) [46, 124, 172]. In the latter, an experimental method to probe the lattice using scanning defect microscopy has been developed [173.

\section{SIMULATING STRONGLY CORRELATED EFFECTS IN 1D WITH PHOTONS IN WAVEGUIDES WITH EIT-BASED NONLINEARITIES}

Simulations of strongly correlated effects with photons are not limited to lattice systems. Continuous one dimensional systems can also be simulated by using nonlinear waveguide systems instead of coupled resonator arrays. This section reviews proposals in this young field. We first explain the mechanism to produce strongly interacting polaritons in 1D waveguides and show how an effective Hamiltonian can be engineered. Equipped with this knowledge, we go on to review various proposals on simulating strongly correlated many-body system in $1 \mathrm{D}$ continuum. These range from the condensed matter models such as the Tonks-Girardeau gas of polaritons, quantum sine-Gordon model, Bose-Einstein condensation of polaritons, photonic Luttinger liquids and Cooper pairing to interacting relativistic model named the Thirring model. We end the section with a brief survey of experimental progress in realizing relevant nonlinear optical setups and a comment on possible future directions.

\section{A. Background}

Quantum simulations of one dimensional quantum field theories with strongly interacting photons (or more precisely, polaritons) to be reviewed in this section rely on two main techniques. The first is the technique of stationary light, allowing one to trap pulses of light and describe its time evolution via an effective Hamiltonian. The second is creating strong nonlinearity, achieved by introducing specifically structured atoms to mediate interaction between photons. Both are closely tied to the phenomenon of electromagnetically induced transparency (EIT) [174 176] as we will explain shortly.

\section{Stationary pulses of light}

The concept of stationary light has been proposed and demonstrated by Bajcsy et al. 177]. The main idea is based on dark state polaritons [178 formed in EIT, which we will briefly explain here. Consider an electric field propagating through an atomic medium. Normally, if the electric field is resonant with an atomic transition, it will be strongly attenuated as it propagates through the medium. However, a laser field driving another transition can induce destructive quantum interference between the excitation pathways and cancel this attenuation, i.e., the extra laser field may induce transparency. In EIT, this is accompanied with a steep dispersion, resulting in a dramatic reduction of the group velocity and enhanced nonlinearity.

Dark state polaritons: Dark state polaritons are collective excitations of light and matter formed when a quantum field propagates under specific conditions that allow EIT. A typical atomic level scheme employed in realizing EIT, called a $\Lambda$-scheme, is depicted in figure 22 . It comprises of a dissipative excited state $|b\rangle$ and two steady states $|a\rangle$ and $|c\rangle$. One transition to the excited state is coupled to a quantum field $\hat{E}$ while the other is coupled to a classical control (laser) field of Rabi frequency $\Omega$. The Hamiltonian describing the evolution of

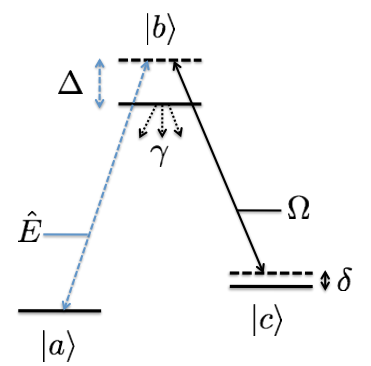

FIG. 22: Atomic level scheme to create dark state polaritons. $|a\rangle$ and $|c\rangle$ are stable states, $\hat{E}$ is a quantum field, and $\Omega$ is the Rabi frequency of a classical control field.

the atom-field system is

$$
\begin{aligned}
H= & -n \int d z\left[\Delta \hat{\sigma}_{b b}+\delta \hat{\sigma}_{c c}+g \sqrt{2 \pi}\left(\hat{\sigma}_{b a} \hat{E}+\text { h.c. }\right)\right. \\
& \left.+\left(\Omega \hat{\sigma}_{b c}+\text { h.c. }\right)\right]
\end{aligned}
$$

where $n$ is the linear density of atoms, $g$ is the single photon-atom coupling strength, and $\hat{\sigma}_{i j} \equiv \hat{\sigma}_{i j}(z, t)$ are continuous average spin-flip operators in a small region around the position $z$. The propagation of the electric field is governed by the equation

$$
\left(\partial_{t}+v \partial_{z}\right) \hat{E}(z, t)=i \sqrt{2 \pi} g n \hat{\sigma}_{a b}(z, t),
$$

where $v$ is the speed of light in the absence of atoms.

Assuming that atoms are mostly in the ground state $|a\rangle$ and adiabatically eliminating the fast-varying atomic coherence $\hat{\sigma}_{a b}$ in the Heisenberg-Langevin equation, the propagation equation reduces to (for a derivation, see for example [179])

$$
\left(\partial_{t}+v_{g} \partial_{z}\right) \hat{\Psi}(z, t)=\frac{i 2 \pi g^{2} n v_{g} \delta}{\left(\Omega^{2}-i \delta \Gamma\right) v} \hat{\Psi}(z, t) .
$$


The field operator $\hat{\Psi}$ describes a collective excitation of the quantum field $\hat{E}$ and the atomic excitation $\hat{\sigma}_{a b}$ which is called the dark state polariton. $\Gamma=\gamma / 2-i \Delta$ where $\gamma$ is the decay rate from the excited state $|b\rangle$ and $v_{g} \approx$ $v \Omega^{2} /\left(\Omega^{2}+2 \pi g^{2} n\right)$ in the limit $\Omega^{2} \gg|\delta \Gamma|$, with $\delta$ the twophoton detuning as depicted in figure 22 The dark state polariton operator reduces to $\hat{\Psi} \approx g \sqrt{2 \pi n} \hat{E}(z, t) / \Omega$ if the energy is mostly stored in the form of atomic excitations, which happens when $2 \pi g^{2} n \gg \Omega^{2}$. In such a regime the group velocity $v_{g}$ is much smaller than the speed of light in the empty waveguide, $v$.

Stationary light: Stationary light is formed when there are two counter-propagating control fields that form a Bragg grating for the quantum fields propagating in the opposite direction. The dark state polaritons are then trapped in the system while maintaining a finite photonic component, following the intensity profile of the control fields. Being stationary, these polaritons have longer time to interact with each other, which effectively enhances the nonlinearity of the system. To derive the equation of motion for these stationary polaritons, we need to first introduce some notations. Counter propagating fields will be denoted as $\hat{E}_{ \pm}$and $\Omega_{ \pm}$, where $\hat{E}=\hat{E}_{+} e^{i k_{c} z}+\hat{E}_{-} e^{-i k_{c} z}$ and $\Omega=\Omega_{+} e^{i k_{c} z}+\Omega_{-} e^{-i k_{c} z} \cdot k_{c}$ is the wavenumber of the control field, which we assume to be similar to the wavenumber of the quantum field. In turn, this induces a slowly varying optical coherence $\hat{\sigma}_{a b, \pm}$ defined through $\hat{\sigma}_{a b}=\hat{\sigma}_{a b,+} e^{i k_{0} z}+\hat{\sigma}_{a b,-} e^{-i k_{0} z}$. Because of the wavenumber mismatch, there is an additional term $i \Delta \omega$ in the r.h.s. of the propagation equation (29), where $\Delta \omega=v\left(k_{0}-k_{c}\right)$ (assuming the same speed of light $v$ for both the quantum and classical light) [180.

If the atoms are hot enough, we can use the secular approximation, i.e., neglect the fast oscillating contributions proportional to $e^{ \pm i 2 k_{0} z}$ to obtain propagation equations similar to 30 for counter propagating dark state polaritons $\hat{\Psi}_{ \pm}=g \sqrt{2 \pi n} \hat{E}_{ \pm} / \Omega_{ \pm}$, but with extra terms that couple the counter propagating fields [177, 179]. When $\Omega_{+}=\Omega_{-} \equiv \Omega_{0}$, these equations can be grouped into the equations for the sum, $\hat{\Psi}_{S}=\left(\hat{\Psi}_{+}+\hat{\Psi}_{-}\right) / \sqrt{2}$, and the difference, $\hat{\Psi}_{D}=\left(\hat{\Psi}_{+}-\hat{\Psi}_{-}\right) / \sqrt{2}$ modes:

$$
\begin{aligned}
\partial_{t} \hat{\Psi}_{S}+2 v_{g} \partial_{z} \hat{\Psi}_{D} & =i\left(\delta-2 \Delta \omega \frac{v_{g}}{v}\right) \hat{\Psi}_{S} \\
\partial_{t} \hat{\Psi}_{D}+v \partial_{z} \hat{\Psi}_{S} & =-\frac{2 \pi g^{2} n}{\Gamma} \hat{\Psi}_{D} .
\end{aligned}
$$

In the limit of interest, $2 \pi g^{2} n \gg \Gamma$, the difference mode $\hat{\Psi}_{D}$ can be adiabatically eliminated to give

$$
i \partial_{t} \hat{\Psi}_{S}=-\frac{1}{2 m_{e f f}} \partial_{z}^{2} \hat{\Psi}_{S}+V_{e f f} \hat{\Psi}_{S}
$$

where $m_{\text {eff }}=-\pi g^{2} n /\left[v v_{g}(\Delta+i \gamma / 2)\right]$ and $V_{\text {eff }}=$ $2 \Delta \omega v_{g} / v-\delta$ can be thought of as a (complex) effective mass and an effective potential in analogy to the Schrödinger equation. Note that the group velocity of the sum mode is zero (it becomes non-zero for non-identical control fields $\Omega_{ \pm}$), indicating that we have a stationary light.

Assuming $\Delta=-|\Delta| \gg \gamma$, the effective mass becomes real and can be written as $\gamma_{1 D} n / 4 v_{g}|\Delta|$, where $\gamma_{1 D}=$ $4 \pi g^{2} / v$ can be identified as the spontaneous emission rate of a single atom into the waveguide. $\eta=\gamma_{1 D} / \gamma$ then defines a 'single-atom cooperativity'-the efficiency of coupling into the waveguide. With these definitions the optical depth of the waveguide is defined as $O D=\eta n l$, where $l$ is the length of the waveguide. $O D$ quantifies the strength of the interaction between photons and the medium and a large value of it $(\approx 1000)$ is typically required to get into the strongly interacting regime considered in this section.

Preparation and detection of stationary pulses of light: Quantum simulations using stationary light largely consists of three steps: 1) loading a traveling pulse, 2) converting it to a stationary pulse that undergoes a prescribed evolution, and 3) detecting the resulting stationary light. Here let us illustrate this with a typical example. A quantum pulse of light $\hat{E}_{+}$is sent in from the left with only the control field $\Omega_{+}$initially turned on. In this typical EIT scheme, the quantum pulse travels slowly with the group velocity $v_{g} \propto \Omega^{2}$. When the pulse has completely entered the medium $\Omega(t)$ is adiabatically turned to zero, trapping the entire pulse in the system [178. Following this loading process, stationary light is created by adiabatically increasing both $\Omega_{+}(t)$ and $\Omega_{-}(t)$ simultaneously, creating the long-lived sum mode $\hat{\Psi}_{S}$ which evolves under an engineered Hamiltonian of choice. After a dynamical evolution or an adiabatic change of the initial state, the pulse is released by turning off $\Omega_{-}$which converts the stationary pulse to a propagating one. The out-going pulse can then be detected using standard quantum optical measurements; for example, a photon correlation measurement can detect spatial correlations of the stationary pulse that have been converted into temporal correlations.

\section{EIT nonlinearities with four-level atoms}

The last essential ingredient in simulating strongly correlated effects with photons is a strong nonlinearity (between photons or more accurately polaritons). Normally, to get photons to interact with eachother strongly, one requires them to be resonant with the atoms, which unfortunately entails strong dissipation. As we have already hinted, an effective way to overcome this problem is to use EIT to suppress the linear absorption while enhancing the Kerr-nonlinearity through a quantum interference effect [176]. However, the problem is that the magnitude of the nonlinearity is limited by dissipation in the usual three-lv scheme.

A scheme that uses four level atoms to generate a giant resonantly enhanced nonlinearities was first introduced in 181, where the atomic structure depicted in figure 23 was shown to yield a cross-Kerr nonlinearity between 
the fields $\hat{E}_{1}$ and $\hat{E}_{2}$. The imaginary part of the thirdorder susceptibility $\chi^{(3)}$ can be made small by increasing the detuning $\Delta_{2}$, while leaving the real part finite and, in fact, orders of magnitude larger than those obtained from the usual 3-lv scheme. Self-Kerr nonlinearity can also be obtained by replacing $\hat{E}_{2}$ with $\hat{E}_{1}$ 66. These types of nonlinearity are exactly what one needs to simulate strongly interacting fields with photons as we will explain in the remainder of this section. We will continue to adopt the Heisenberg-Langevin equation approach to study the dynamics in the low-loss regime in this review, but a master equation description can also be employed as shown by Kiffner and Hartmann [182.

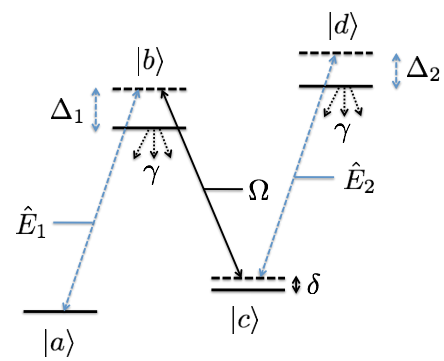

FIG. 23: Atomic level scheme to generate a giant resonantlyenhanced Kerr nonlinearity.

\section{B. Tonks-Girardeau gas of photons}

Combining the techniques of stationary light and giant Kerr nonlinearity, Chang et al. 49] showed that photons can crystallize and mimic the behaviour of strongly interacting one-dimensional bosons called the TonksGirardeau (TG) gas 116, 183. The system consists of an ensemble of four-level atoms interfaced with a tightconfining waveguide such as a tapered optical fiber or a hollow-core optical fiber. There has also been a proposal to observe a TG gas induced by a purely-dissipative interaction [184] in the same setting [185, but we will not discuss it here. Let us start with a gentle introduction to the physics of 1 dimensional Bose gas before delving into the proposal of Chang and coworkers.

Studies have shown that In 1 (spatial) dimension, effects of quantum fluctuations are enhanced and interacting particles show behaviour dramatically different from the higher dimensional analogues 186. A famous example is the TG gas, where strongly interacting bosons are effectively fermionized. Dynamics of the simplest interacting bosons in 1D are governed by the Lieb-Liniger model [187], described by the Hamiltonian

$$
-\int d z \Psi^{\dagger}(z) \frac{1}{2 m} \partial_{z}^{2} \Psi(z)+U \Psi^{\dagger}(z) \Psi^{\dagger}(z) \Psi(z) \Psi(z)
$$

which for a fixed linear density $n$ is characterized by a single dimensionless parameter $\gamma_{L L}=n U /\left(n^{2} / 2 m\right)$. When
$\gamma_{L L} \gg 1$, the bosons are impenetrable, which means that there is an effective exclusion principle and the system behaves in many ways like a non-interacting Fermi gas. This fermionization can be observed in the secondorder density-density correlation function $g^{(2)}\left(z, z^{\prime}\right)=$ $\left\langle I(z) I\left(z^{\prime}\right)\right\rangle /\left(\langle I(z)\rangle\left\langle I\left(z^{\prime}\right)\right\rangle\right)$, for example, as pointed out by Chang and coworkers. The TG gas has been realized with cold atoms in optical lattices [188, 189, but a realization with photons brings in a new set of techniques such as the standard photon-correlation measurements.

A schematic diagram of the proposed setup to realize a TG gas of photons is depicted in figure 24. The two-photon detuning $\delta$ is assumed to be zero and each atomic coherence is coupled to two counter-propagating fields as in the stationary light setting. Furthermore, the atomic transition $c \leftrightarrow d$ is coupled to the same quantum fields as the transition $a \leftrightarrow b$ so that the self-Kerr nonlinearity is induced. In the absence of the fourth lv $|d\rangle$, we have already seen that the sum mode obeys the Schrödinger equation (33). With the fourth level, this equation becomes a nonlinear Schrödinger equation (assuming $\left.V_{\text {eff }}=0\right)$ 49

$$
i \partial_{t} \hat{\Psi}(z, t)=-\frac{1}{2 m_{e f f}} \partial_{z}^{2} \hat{\Psi}(z, t)+2 \tilde{g} \hat{\Psi}^{\dagger}(z, t) \hat{\Psi}^{2}(z, t),
$$

where $m_{e f f}=-\pi g^{2} n /\left[v v_{g}\left(\Delta_{1}+i \gamma / 2\right)\right]$ as before and $2 \tilde{g}=2 \pi g^{2} v_{g} /\left[\left(\Delta_{2}+i \gamma / 2\right) v\right]$. We have assumed for convenience that the two excited states have the same decay rate $\gamma$. Because the dark state polariton operators obey the bosonic commutation relations, the above equation describes the evolution under the Lieb-Liniger Hamiltonian (34). In particular, the dimensionless parameter $\gamma_{L L}$ is written in terms of bare optical parameters as

$$
\gamma_{L L}=\frac{m_{e f f} \tilde{g}}{n_{p h}}=-\frac{\pi^{2} g^{4}}{\left(\Delta_{1}+i \gamma / 2\right)\left(\Delta_{2}+i \gamma / 2\right)} \frac{n}{n_{p h}},
$$

where $n_{p h}$ is the linear density of photons at the centre of the pulse. Notice that there is a non-zero imaginary part, which will be neglected on the basis that $\left|\Delta_{1,2}\right| \gg \gamma$.

Among the characteristic features of a TG gas is an oscillating density-density correlation function, similar to the Friedel oscillations in electronic systems with an impurity 190. In the TG limit, the second-order intensity correlation function $g^{(2)}\left(z, z^{\prime}\right)=$ $\left\langle I(z) I\left(z^{\prime}\right)\right\rangle /\left(\langle I(z)\rangle\left\langle I\left(z^{\prime}\right)\right\rangle\right)$ of the ground state takes the form 191]

$$
g_{\mathrm{TG}}^{(2)}\left(z, z^{\prime}\right)=1-\left(\frac{\sin k_{F}\left(z-z^{\prime}\right)}{k_{F}\left(z-z^{\prime}\right)}\right)^{2},
$$

where $I(z)=\hat{\Psi}^{\dagger}(z) \hat{\Psi}(z)$ and $k_{F}=\pi n_{p h}$ is the 'Fermi momentum' of the photonic TG gas.

The $2 k_{F}$ oscillations can be observed experimentally by following the preparation and detection procedure outlined in subsection IV A 1. An initial pulse of coherent state is loaded into the waveguide and turned into a stationary pulse. Then the dimensionless interaction parameter $\gamma_{L L}$ is increased from zero to a large value by 


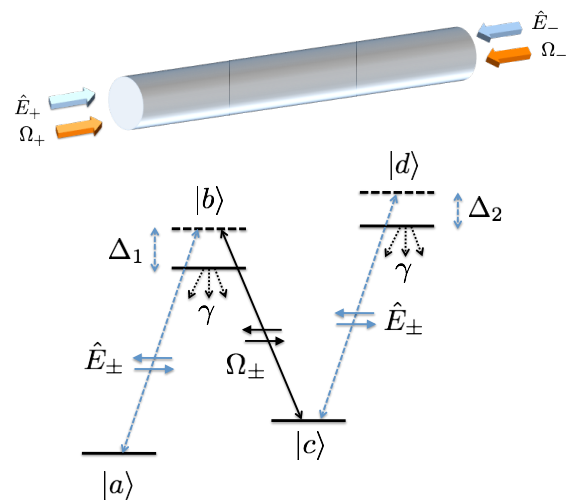

FIG. 24: Nonlinear waveguide setup simulating the LiebLiniger model with stationary pulses of light. The waveguide contains 4-lv atoms whose coupling scheme with the quantum and control fields is indicated.

changing, for example, the detuning. The initial coherent state, which is an eigenstate of the free Hamiltonian, remains in an eigenstate of the Hamiltonian (with non-zero interaction strength) and therefore exhibits the Friedel oscillations. The stationary pulse can then be converted to a traveling pulse, whose temporal correlation function reveals the density-density correlation function of the simulated quantum state.

An example is shown in figure 25, taken from [49, for an initial density profile $n_{p h}(z)=n_{0}\left(1-z^{2} / z_{0}^{2}\right)^{1 / 2}$ under an exponentially increasing interaction parameter $\gamma_{L L}$. The distance is in units of the inverse effective Fermi wavevector $k_{F}$ and the final time is $t=10 \omega_{f}^{-1}$, where $\omega_{f}$ is the Fermi energy on the order of $n_{p h}^{2} / m_{e f f}$. For an optical depth of $\approx 2000$ and the cooperativity $\eta \approx 0.2$, the achievable maximum $\gamma_{L L}$ is around 10 .

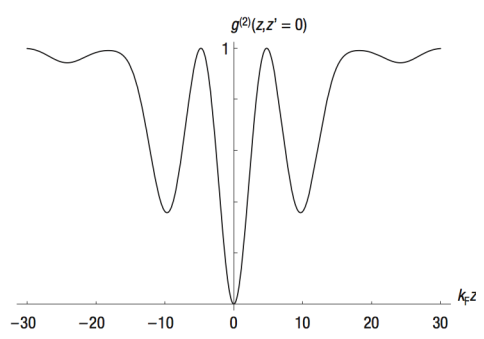

FIG. 25: Density-density correlation function $g^{(2)}\left(z, z^{\prime}=0\right)$ of the final TG gas of photons. Initial density profile is $n_{p h}(z)=n_{0}\left(1-z^{2} / z_{0}^{2}\right)^{1 / 2}, N_{p h}=10, z_{0}=5 k_{F}^{-} 1$, and $t=10 \omega_{f}^{-1}$. Reprinted with permission from Chang et al. 49.
C. Introducing an effective potential-Bose Hubbard and sine-Gordon models, and the 'pinning' transition

Looking back to equation (33), it is not too difficult to see that a spatially dependent two-photon detuning would induce an effective potential for the stationary light. Indeed, this fact has been noticed in a proposal to simulate the Klein tunnelling with stationary light 22 . Upon introducing another transition, one can induce a small spatially-dependent two-photon detuning and as a consequence the resulting nonlinear Schrödinger equation would have an effective potential term. Another method is to induce a small change in the spatial profile of the atomic density [192. The latter requires cold atomic gas so that the density profile does not change appreciably during the experiment. However, evolution of a stationary light in a cold atomic ensemble is different to that in a hot vapour because of the secular approximation discussed earlier in subsection IV A 1, 193, 194] and one has to resort to a different atomic level scheme of a doublelambda type. Here, we discuss the effects of introducing a lattice potential to strongly interacting photons assuming that the lattice has been created via one of the two methods and defer a discussion on stationary light in cold atomic gases to the next subsection.

We follow Huo and Angelakis 192 and consider the Lieb-Liniger model with an additional potential term of the form $V(z)=V_{0} \cos ^{2}\left(\pi n_{p h} z\right)$. The periodicity of the lattice potential is set such that the number of sites equals the number of photons in the waveguide. Depending on the lattice depth and the interaction strength, the system shows either the Mott insulating phase or the superfluid phase [195] as depicted schematically in figure 26 .

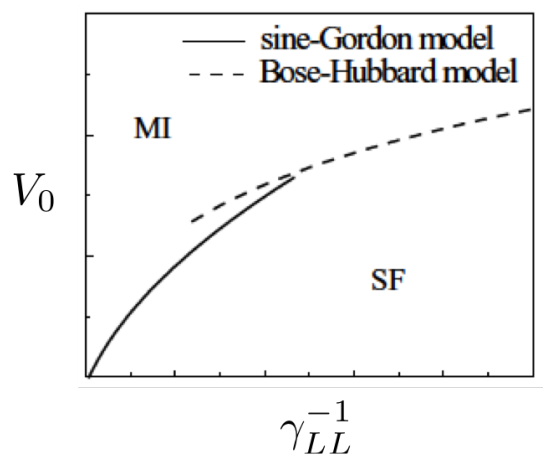

FIG. 26: A figure showing the phase diagram of the LiebLiniger model in the presence of a potential. The interaction strength and the lattice depth is changed by tuning an optical parameter which is not specified here. Reprinted from Huo and Angelakis [192.

There are two interesting limits in the phase diagram: i) weakly interacting particles $\left(\gamma_{L L}<1\right)$ in a deep potential $\left(V_{0} \gg E_{r}\right)$ and ii) strongly interacting particles $\left(\gamma_{L L}>1\right)$ in a shallow potential $\left(V_{0} \sim E_{r}\right)$, where 
$E_{r}=\pi^{2} n_{p h}^{2} / 2 m_{e f f}$ is the recoil energy. For case i), the physics is well described by the well-known BoseHubbard model [71, 196, where the lowest localised Wannier mode in each lattice site is coupled to the modes in its nearest neighbour sites with depth-dependent on-site interaction strength. As we have seen earlier, the BoseHubbard hamiltonian reads

$$
H_{B H}=-J \sum_{\langle i, j\rangle} a_{i}^{\dagger} a_{j}+\frac{U}{2} \sum_{i} a_{i}^{\dagger} a_{i}^{\dagger} a_{i} a_{i}
$$

where $a_{i}$ is now interpreted as the annihilation operator for the Wannier mode at site $i$, $\sqrt{\pi} J / E_{r}=4\left(V_{0} / E_{r}\right)^{3 / 4} e^{-2 \sqrt{V_{0} / E_{r}}}$, and $U / E_{r}=$ $\sqrt{2 / \pi^{3}}\left(V_{0} / E_{r}\right)^{1 / 4} \gamma_{L L}$. Note that the effective parameters $J$ and $U$ only depend on $V_{0}, E_{r}$, and $\gamma_{L L}$, all of which can be tuned by changing quantum optical parameters. By tuning the value of $U / E_{r}$, through changing the value of the detuning $\Delta_{2}$ for example, one can observe the Mott-to-superfluid transition.

There is also an interesting quantum phase transition called a 'pinning' transition in case ii), when the interaction is strong. In the weak lattice limit, the particle density is weakly modulated and one is able to separate out the density perturbation and phase as canonically conjugate observables. Haldane was the first to consider this case and show that the quantum sine-Gordon model correctly describes the physics in this limit [197. Following the work of Büchler, Blatter, and Zwerger [195], we briefly reproduce the derivation of the sine-Gordon Hamiltonian here.

First consider a hydrodynamic approximation where one describes the bosonic field operator in the low energy limit as $\Psi \approx \sqrt{\rho_{0}+\partial_{z} \theta / \pi} \exp (i \phi)$, where $\rho_{0}$ is the mean density of the atoms, $\partial_{z} \theta / \pi$ is the long-wavelength density fluctuation, and $\phi$ is the phase field. The latter two obeys the boson commutation relation $\left[\partial_{z} \theta(z), \phi\left(z^{\prime}\right)\right]=$ $i \pi \delta\left(z-z^{\prime}\right)$. Neglecting the lattice potential for now, the low-energy physics is described by the Hamiltonian

$$
H_{0}=\frac{1}{2 \pi} \int d z\left[v_{J}\left(\partial_{z} \phi\right)^{2}+v_{N}\left(\partial_{z} \theta\right)^{2}\right] .
$$

The first part comes from the kinetic energy where $v_{J}=$ $\pi \rho_{0} / m$ and the second term derives from the interaction energy with the coefficient $v_{N}=\partial_{n} \mu / \pi$ determined by the inverse compressibility. To obtain the Hamiltonian due to the lattice potential, one needs to modify the density operator to account for its discrete character:

$$
\rho(z)=\left[\rho_{0}+\frac{1}{\pi} \partial_{z} \theta\right]\left[1+2 \sum_{l=1}^{\infty} \cos \left(l \theta+l \pi \rho_{0} z\right)\right] .
$$

In the commensurate limit (one particle per lattice cell), the dominant part of the potential arising from the lowest harmonic in the above equation takes the sine-Gordon form

$$
H_{V}=\frac{n_{p h} V_{0}}{2} \int d z \cos (2 \theta)
$$

The Hamiltonian $H_{0}+H_{V}$ is the 1 dimensional quantum sine-Gordon equation which is known to exhibit the so-called 'pinning' transition 198. This phase transition can best be described by the dimensionless parameter $K=\sqrt{v_{J} / v_{N}}$ that is related, in the absence of the periodic potential, to the Lieb-Liniger parameter $\gamma_{L L}$; $K(\gamma \leq 10) \approx \pi / \sqrt{\gamma_{L L}-(1 / 2 \pi) \gamma_{L L}^{3 / 2}}$ and $K(\gamma \gg 10) \approx$ $\left(1+2 / \gamma_{L L}\right)^{2}$. When $K>K_{c}=2$, the weak potential is irrelevant and the bosons are in the superfluid phase whereas for $K<K_{c}$ an arbitrarily weak potential can pin the bosons to lattice sites, achieving the Mott insulating state.

The procedure to observe these phases in the nonlinear waveguide system with a lattice potential is as follows. First a pulse containing a certain number of photons is loaded into the waveguide. Then one turns up the interaction strength and then the lattice potential adiabatically. This step can be realized experimentally by changing the detunings $\Delta_{2}$ and $\delta$. By the end of this step, the photonic state would have adiabatically followed the Hamiltonian and consequently would exhibit either the superfluid or the Mott-insulating behaviour which can be observed by releasing the stationary pulse and performing correlation measurement on them as in the observation of a TG gas of photons.

\section{Bose-Einstein condensation of stationary-light polaritons}

The realization of strongly interacting photons in the previous subsection relied on having a hot vapour of atoms so that the secular approximation holds. However, it is also possible to create stationary light with cold atoms [199, 200] as demonstrated in 201]. In particular, Zimmer et al. 200] showed how the linear Schrödinger equation can be obtained with cold atoms using a slightly different atomic level scheme shown in figure 27(a). In this scheme, $\hat{E}_{+}$and $\hat{E}_{-}$couple to different atomic transitions unlike in the usual $\Lambda$ scheme where the two couple to the same transition, so the secular approximation no longer needs to be invoked. For equal control field strengths, $\Omega_{+}=\Omega_{-}$, the stationary dark state polariton is defined as

$$
\hat{\Psi}(\mathbf{r}, t)=\frac{\cos \theta}{\sqrt{2}}\left(\hat{E}_{+}(\mathbf{r}, t)+\hat{E}_{-}(\mathbf{r}, t)\right)-\sin \theta \hat{S}(\mathbf{r}, t),
$$

where $\tan ^{2} \theta=2 \pi g^{2} n / \Omega^{2}, \Omega^{2}=\Omega_{+}^{2}+\Omega_{-}^{2}$, and $\hat{S}=$ $\sqrt{n} \hat{\sigma}_{a c}(\mathbf{r}, t)$. The Schrödinger equation obeyed by this field operator reads

$$
i \partial_{t} \hat{\Psi}(\mathbf{r}, t)=-\left[\frac{1}{2 m_{\|}} \partial_{z}^{2}+\frac{1}{2 m_{\perp}} \Delta_{\perp}\right] \hat{\Psi}(\mathbf{r}, t),
$$

where $m_{\perp}=m v_{r e c} / v_{g}$ and $m_{\|}^{-1}=$ $m_{\perp}^{-1} k_{p}\left(\gamma v / 2 \pi g^{2} n\right)(2 \Delta / \gamma+i)$. Here $k_{p}$ is the wavenumber 
(a)

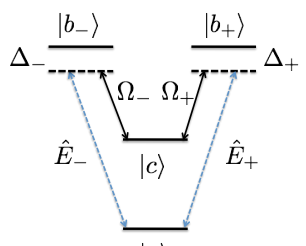

$|a\rangle$ (b)

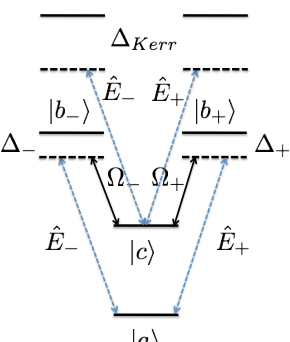

$|a\rangle$

FIG. 27: (a) Atomic level scheme for creating dark state polaritons using cold atoms. (b) Modified atomic level scheme to induce the Kerr interaction between dark state polaritons, allowing for them to Bose condense.

of the probe field and $v_{r e c}=k_{p} / m$ is the recoil velocity of an atom of mass $m$.

Introduction of additional levels as in figure 27(b) adds the Kerr-type interaction between the dark state polaritons whose elastic collision rate can be much greater than the loss rates and thereby allow thermalization of polaritons without significant losses 202. The optical depth required to reach this regime is only modestly high at about $O D>30$. For realistic parameters, the critical temperature to create a BEC out of dark state polaritons (as compared to the critical temperature for ideal bosonic atoms) is $T_{c} / T_{c}^{a t o m s} \approx 6 \times 10^{5}$ which is in the $m K$ regime. The latter is in the same range as the temperature limit of about $1 \mathrm{mK}$ imposed by the finite frequency window of EIT. Therefore, it is possible to observe the BEC of stationary light polaritons at temperatures several orders of magnitude higher than the critical temperature of atomic BECs.

In the stationary light setup, a BEC will be created not by reducing the temperature of the polariton gas, but by increasing the critical temperature of condensation through changing the effective mass. The steps to observe Bose-Einstein condensation of polaritons is then as follows. i) A pulse of coherent light is trapped as stationary polaritons using the same method described in the previous subsection. Initially, the critical temperature is below the ambient temperature so there is no condensation. ii) As the critical temperature is increased, by tuning the strength of the control laser, the polaritons thermalize through elastic collisions and reach BEC before significant losses occur. This causes the macroscopic occupation of $\mathbf{k}=0$ momentum state, which can be iii) observed by converting the stationary polariton into a traveling one, whose transverse profile shows the onset of BEC. The latter is illustrated in figure 28, where the transverse emission profiles are shown at temperatures above (left) and below (right) the critical temperature. A similar proposal with Rydberg atoms-to induce dipolar interaction between dark state polaritons-have been made by Nikoghosyan et al. 203.
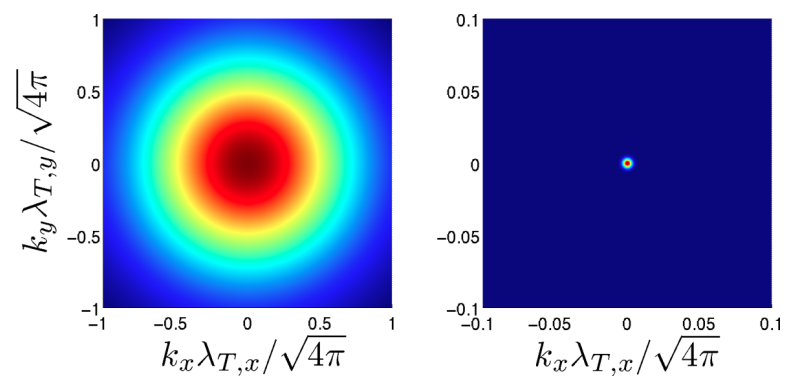

FIG. 28: Transverse emission profile above (left) and below (right) the condensation temperature in arbitrary units, assuming an ideal quasi-homogeneous Bose-gas of width $178 \lambda_{T, x}$, where $\lambda_{T, x}$ is the transversal de Broglie wavelength at temperature $T$. Reprinted with permission from Fleischhauer et al [202].

E. Two-component bosonic models: Luttinger liquids, spin-charge separation and Cooper pairing with photons

So far in this section, we have reviewed works on quantum simulations of strongly interacting bosons when there is only a single species of bosons. Soon after the pioneering work on a TG gas of stationary light, Angelakis et al. realized that the physics of two interacting bosons can also be simulated in a similar waveguide setup [50, 204. Here, we describe how this leads to a realization of the two-component Lieb-Liniger model, which could allow a clear experimental observation of spin-charge separation. Furthermore, by introducing a lattice potential, one can also simulate a two-component Bose-Hubbard model, which exhibits interesting behaviours such as (a 1D analogue of) the famous BEC-BCS crossover [205, 206].

A schematic diagram of the atomic level scheme that yields the two-component Lieb-Liniger model is shown in figure 29. Two different quantum fields $\hat{E}_{1}$ and $\hat{E}_{2}$ with frequencies $\omega_{q u}^{(1)}$ and $\omega_{q u}^{(2)}$ interact with two different species of 4-lv atoms labeled $a$ and $b$ and forms two polariton operators via the usual relation $\hat{\Psi}_{j, \pm}=$ $g \sqrt{2 \pi n^{x_{j}}} \hat{E}_{j, \pm} / \Omega_{x_{j}}$. The figure depicts the level scheme for type- $a$ atoms; atoms of type $b$ have a similar coupling scheme except that the roles of $\hat{E}_{1}$ and $\hat{E}_{2}$ are reversed and the detunings are accordingly labeled by replacing $a$ with $b$. We use $j=1,2$ to denote the polariton species, $x_{1}=a, x_{2}=b$ to label the atomic species, and we have assumed that the atom-photon coupling strength is independent of the atomic species and transition. $n^{a}\left(n^{b}\right)$ denotes the density of atomic species $a(b)$ and $\delta_{i j}=\omega_{q u}^{(i)}-\omega_{q u}^{(j)}$. By setting the detunings such that $\hat{E}_{1}\left(\hat{E}_{2}\right)$ is only coupled to atoms of type- $b(a)$ through the transition $|3\rangle \leftrightarrow|4\rangle$, one obtains a cross-species interaction term on top of the self-interaction terms. The dynamics of the polaritons are thus governed by the two- 
component Lieb-Liniger model

$$
\begin{aligned}
H_{\mathrm{tcll}} & =\int d z \sum_{j}\left[-\frac{1}{2 m_{j}} \hat{\Psi}_{j}^{\dagger}(z) \partial_{z}^{2} \hat{\Psi}_{j}(z)\right. \\
& \left.+\frac{U_{j}}{2} \hat{\Psi}_{j}^{\dagger}(z) \hat{\Psi}_{j}^{\dagger}(z) \hat{\Psi}_{j}(z) \hat{\Psi}_{j}(z)\right]+\frac{V}{2} \rho_{1}(z) \rho_{2}(z),
\end{aligned}
$$

where $\rho_{j}=\hat{\Psi}_{j}^{\dagger}(z) \hat{\Psi}_{j}(z) \cdot m_{j}$ and $U_{j}$ are defined as $m_{e f f}$ and $4 \tilde{g}$ introduced earlier, with species-dependent optical parameters, whereas the inter-species interaction parameter $V$ is new. For simplicity, we will assume that all species-dependence are suppressed. Then the interspecies interaction strength is compactly written as

$$
V=\frac{16 \pi g^{2} n v_{g}}{\Delta_{2}}
$$

An alternative scheme where the two species correspond to different polarizations has also been proposed in [204]. We will come back to this setup in Sect. IVF where a quantum simulation of an interacting relativistic model will be reviewed.

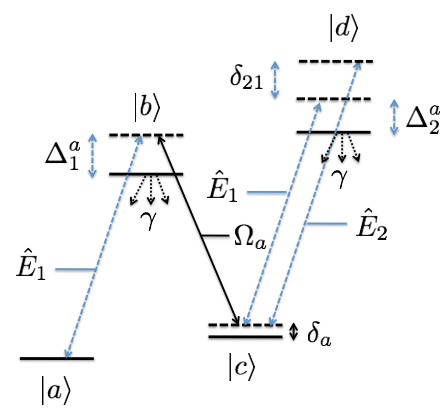

FIG. 29: Atomic level scheme to realize the two-component Lieb-Liniger model, for the type- $a$ atoms. Type- $b$ atoms have the role of $\hat{E}_{1}$ and $\hat{E}_{2}$ reversed and the super- and sub-script $a$ replaced by $b$, as well as $\delta_{21} \rightarrow \delta_{12}$.

\section{Luttinger liquid and spin-charge separation}

We have already seen how Lieb-Liniger bosons can be described, in the low-energy sector, in terms of the long-wave density function $\partial_{z} \theta$ and the phase field $\phi$. It turns out that the same decomposition applied to the two-component Lieb-Liniger model separates the Hamiltonian into two independent components, describing spin and charge components that travel in different speeds. Let us assume that the two species have identical parameters, i.e., $m_{j}=m, U_{j}=U$, and $\left\langle\rho_{j}\right\rangle=\rho$. Following the notations in [207] (see also 208]), the Hamiltonian (44) becomes $H=H_{c}+H_{s}$, where

$$
H_{c}=\frac{1}{2 \pi} \int d z v_{c} K_{c}\left(\partial_{z} \theta_{c}\right)^{2}+\frac{v_{c}}{K_{c}}\left(\partial_{z} \phi_{c}\right)^{2}
$$

and

$$
\begin{aligned}
H_{s} & =\frac{1}{2 \pi} \int d z v_{s} K_{s}\left(\partial_{z} \theta_{s}\right)^{2}+\frac{v_{s}}{K_{s}}\left(\partial_{z} \phi_{s}\right)^{2} \\
& +\frac{V}{\left(\pi \rho^{-1}\right)^{2}} \cos \left(\sqrt{8 \phi_{s}}\right)
\end{aligned}
$$

Here the subscripts $c$ and $s$ refer to the charge and spin components and $v_{c, s}=v_{0} \sqrt{1 \pm V K / \pi v_{0}}$ and $K_{c, s}=$ $K / \sqrt{1 \pm V K /\left(\pi v_{0}\right)}$, where analytic expressions $v_{0}=$ $\sqrt{U \rho / m}$ and $K=\sqrt{\pi^{2} \rho /(m U)}$ hold in the weakly interacting regime.

The physics of this system is fully determined by the velocities $v_{c, s}$ and the so-called Luttinger parameters $K_{c, s}$. Note that the speed of the spin and charge degrees of freedom can be different and could even have different signs. In terms of polaritons, the spin and charge densities correspond to the difference and the sum of the densities of two stationary polariton components. Therefore one could create an excitation in the original polariton field and observe as it splits into two components. One would go through the preparation stage discussed before, then let the two components evolve under different speeds, followed by the conversion into traveling slow-light which can be detected via standard optical measurements and post-processed to determine the velocities of the spin and charge parts, for example. In [50, it was shown that the optical depth of $\approx 3000$ is sufficient to create the difference $v_{c} / v_{s}=2$ and observe spin-charge separation by measuring the single particle spectral function, which can be determined from the first order correlation function of one of the fields, say $\hat{E}_{1}$ at a certain quasi-momentum.

\section{BCS-BEC like crossover}

Introducing an effective lattice potential to the twocomponent setup, one can also experimentally realize a 1 dimensional two-component Bose-Hubbard model [206], which exhibits some interesting properties. For example, upon inducing strong repulsive interaction between the same species, one can go into an effective Fermi-Hubbard regime where a 1 dimensional analogue of the famous BCS-BEC crossover can be observed. This requires an attractive inter-species interaction, which is easily achieved in the stationary light setup by changing the sign of a single-photon detuning. In the strongly attractive case, the two species tend to pair up to form a molecule although no more than a pair is allowed at a single site because of the effective Pauli exclusion due to the strong intra-species repulsion. This is reminiscent of the shortrange-correlated bosonic molecules forming in the BEC regime. In the weakly attractive case, two species tend to stay away from each other and form a long-range correlated pair analogous to the Cooper pair. Thus, by continuously tuning the inter-species interaction strength, it is possible to observe a 1 dimensional analogue of the 
BEC-BCS cross-over. A schematic illustration of these phases is provided in figure 30 .
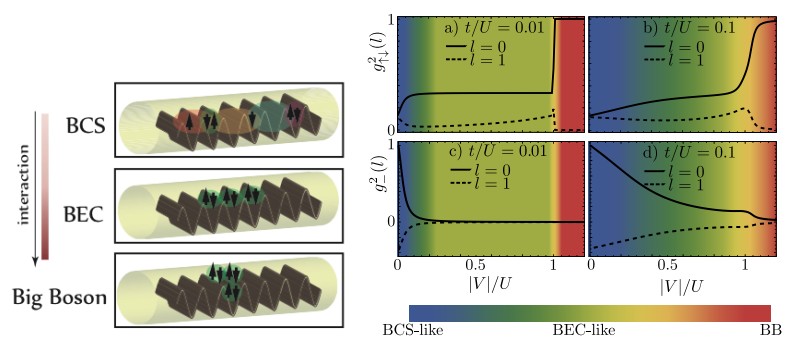

FIG. 30: Left: A schematic representation of the three regimes achievable in the system for different inter-species interaction strength. A crossover from a BCS-like to a BEClike phase occurs for small inter-species strengths compared to the intra-species repulsion, whereas in the opposite limit all the stationary polaritons gather at one site to form the socalled Big-Boson state. Right: Cross-species density-density correlation function, $g_{\uparrow \downarrow}^{2}$, and the density-density correlations between the cross-species population difference, $g_{-}^{2}$, as functions of the inter-species interaction strength. The left column is deeper into the effective Fermi-Hubbard regime compared to the right column, displaying sharper crossovers and transitions. Reprinted from Huo et al. 206].

An optical depth of approximately 1000 was shown to be sufficient for simulating the crossover, where tuning the frequency difference between the quantum fields changes the ratio between the inter- and intra-species interaction strengths. Going through the standard preparation and release processes, one could measure the cross-species intensity correlation function to clearly distinguish all three (BCS-like, BEC-like, and the BigBoson) phases. In figure 30 we plot two types of correlation functions: $g_{\uparrow \downarrow}^{2}(l)=\sum_{i}\left\langle n_{i \uparrow} n_{i+l \downarrow}\right\rangle$ and $g_{-}^{2}(l)=$ $\sum_{i}\left\langle\left(n_{i \uparrow}-n_{i \downarrow}\right)\left(n_{i+l \uparrow}-n_{i+l \downarrow}\right)\right\rangle$. The same-site crossspecies density-density correlation function $g_{\uparrow \downarrow}^{2}(0)$ clearly shows the cross-over behaviour upon changing the interspecies interaction strength, whereas a sharp transition is observed as the inter-species attraction becomes stronger than the intra-species repulsion. Correlations in the population difference, $g_{-}^{2}$, is sensitive to the BCS-BEC cross over, but not the transition to the Big-Boson phase.

\section{F. Interacting relativistic theories with slow light}

Simulations of quantum field theories using nonlinear waveguides can go beyond non-relativistic models. In particular, it was shown that the Thirring model (TM) 209, a relativistic quantum field theory describing the self-interaction of a Dirac field in 2 spacetime dimensions, can be implemented [51]. The atomic level scheme required is shown in figure 31(a). It consists of two sets of the standard four-level arrangements $\left\{|a\rangle,|b, s\rangle,|c, s\rangle,\left|d, s, s^{\prime}\right\rangle\right\}$, with $s, s^{\prime}=\{\uparrow, \downarrow\}$ denoting the two oppositely-polarized quantum pulses $\hat{E}_{\uparrow}$ and
$\hat{E}_{\downarrow}$. Two self-interacting stationary polaritons $\hat{\Psi}_{s}$ appear in exactly the same manner as discussed earlier and interact through the extra level $|d, s, \bar{s}\rangle, \bar{s} \neq s$, with strength dependent on $\Delta_{s, s^{\prime}}$. Making the usual assumptions, these polaritons can be shown to obey the twocomponent Lieb-Liniger model 204. To allow for the linear momentum term required in relativistic models, a small but finite difference between the forward and backward propagating control fields is necessary. Accommodating this change, the (quasi-) stationary lights are now defined as $\hat{\Psi}_{s}=\alpha_{s,+} \hat{\Psi}_{s,+}+\alpha_{s,-} \hat{\Psi}_{s,-}$ with $\alpha_{s, \pm}=$ $\Omega_{s, \pm}^{2} /\left(\Omega_{s,+}^{2}+\Omega_{s,-}^{2}\right)$ and $\hat{\Psi}_{s, \pm}=\sqrt{2 \pi n} g \hat{E}_{s, \pm} / \Omega_{s, \pm}$. The dynamics of these fields are governed by

$$
\begin{aligned}
i \partial_{t} \hat{\Psi}_{s} & =-\frac{1}{2 m_{n r, s}} \partial_{z}^{2} \hat{\Psi}_{s}+i \eta_{s} \partial_{z} \hat{\Psi}_{s}+\Omega_{0} \hat{\Psi}_{\bar{s}} \\
& +\chi_{s s} \hat{\Psi}_{s}^{\dagger} \hat{\Psi}_{s}^{2}+\chi_{s \bar{s}} \hat{\Psi}_{\bar{s}}^{\dagger} \hat{\Psi}_{\bar{s}} \hat{\Psi}_{s}
\end{aligned}
$$

where $m_{n r, s}=-\bar{\Omega}_{s}^{2} /\left(4 v_{s}^{2} \Delta_{s} \sin ^{2} 2 \varphi_{s}\right), \eta_{s}=-2 v_{s} \cos 2 \varphi_{s}$, $\chi_{s s}=4 \bar{\Omega}_{s}^{2} /\left(\Delta_{s s} n\right)$, and $\chi_{s \bar{s}}=2 \bar{\Omega}_{s}^{2}\left[2+\cos \left(\varphi_{\bar{s}}-\right.\right.$ $\left.\left.\varphi_{s}\right)\right] /\left(\Delta_{s \bar{s}} n\right)$. $\quad v_{s}$ is the reduced group velocity for the dark state polaritons, $\varphi_{s}$ is determined from the relation $\tan ^{2} \varphi_{s}=\Omega_{s,-}^{2} / \Omega_{s,+}^{2}$, and $\bar{\Omega}_{s}^{2}=\left(\Omega_{s,+}^{2}+\Omega_{s,-}^{2}\right) / 2$, and $\Omega_{0}$ is the coupling rate between $|c, \uparrow\rangle$ and $|c, \downarrow\rangle$ as shown in figure 31(a).

(a)
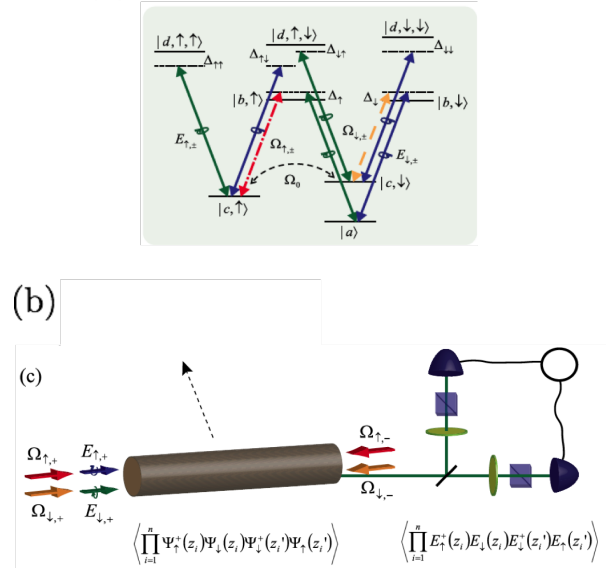

FIG. 31: (a) Proposed atomic level scheme to realize the Thirring model using two polarized stationary light pulses. (b) Experimental setup illustrating photon correlation measurements at the output that facilitates the direct measurement of the n-point correlation functions. Reprinted from Angelakis et al. [51.

To transform the above equation into the bosonic TM, we need to throw away the quadratic dispersion term and set $\eta_{\uparrow}=-\eta_{\downarrow}=\eta$. The latter can be achieved by setting $v_{\uparrow}=v_{\downarrow}$ and $\cos 2 \varphi_{\uparrow}=-\cos 2 \varphi_{\downarrow}$, whereas the former requires the non-relativistic kinetic energy to be much smaller than the relativistic kinetic energy, which can be achieved by an appropriate tuning of the single photon detunings $\Delta_{s}$. Defining a spinor field $\boldsymbol{\Psi}=\left(\hat{\Psi}_{\uparrow}, \hat{\Psi}_{\downarrow}\right)^{T}$, 
the Hamiltonian generating the equation can be written as

$$
\begin{aligned}
H & =\int d z \overline{\mathbf{\Psi}}\left(-i|\eta| \gamma_{1} \partial_{z}+m_{0} \eta^{2}\right) \mathbf{\Psi} \\
& +\sum_{s} \frac{\chi_{s s}}{2} \hat{\Psi}_{s}^{\dagger} \hat{\Psi}_{s}^{\dagger} \hat{\Psi}_{s} \hat{\Psi}_{s}+\frac{\chi}{2} \overline{\mathbf{\Psi}} \gamma^{\mu} \boldsymbol{\Psi} \overline{\mathbf{\Psi}} \gamma_{\mu} \boldsymbol{\Psi},
\end{aligned}
$$

where $\overline{\mathbf{\Psi}}=\boldsymbol{\Psi}^{\dagger} \gamma_{0}$ with the gamma matrices defined as $\gamma_{0}=\gamma^{0}=\sigma_{x}, \gamma_{1}=-\gamma^{1}=i \sigma_{y}$ and $m_{0}=-\Omega_{0} / \eta^{2}$. Thus, neglecting the small quadratic dispersion term, the dynamics of the stationary polaritons is governed by the bosonic TM. Furthermore, by entering the hardcore regime in which the self-interaction energy dominates over all other terms, one can get into the fermionic regime where, as discussed earlier, the one-dimensional bosons behave in many ways as fermions. Figure 32 illustrates that this regime (small kinetic energy + hardcore) can be reached in a possible experiment, assuming achievable values for the optical parameters: $\left|\cos 2 \varphi_{s}\right|=0.004$, $n=10^{7} m^{-1}, \bar{\Omega}_{2} \approx 1.5 \gamma, 0<\chi /|\eta|<\pi$, and the single atom cooperativity $\gamma_{1 D} / \gamma=0.2$.
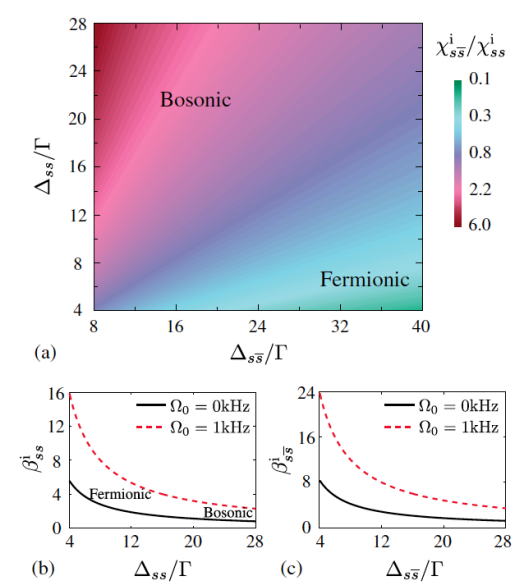

FIG. 32: (a) The ratio of inter- to intra-species interaction strength as a function of two experimentally controllable detunings for $m_{0}=0$. (b) and (c): The ratio of intra- and inter-species interaction energy to kinetic energy, respectively. Reprinted from Angelakis et al. [51].

One of the methods to extract interesting physical information in field theories is to calculate correlation functions. In the massless fermionic TM, the n-point correlation function defined as $\left\langle 0\left|\prod_{i=1}^{n} \hat{\Psi}_{\uparrow}^{\dagger}\left(z_{i}\right) \hat{\Psi}_{\downarrow}\left(z_{i}\right) \hat{\Psi}_{\downarrow}^{\dagger}\left(z_{i}^{\prime}\right) \hat{\Psi}_{\uparrow}\left(z_{1}^{\prime}\right)\right| 0\right\rangle$ has been explicitly calculated 210, and serves as an excellent check for the quantum simulator. After a satisfactory agreement has been obtained in the massless case, one can increase the value of the mass and explore the correlation functions in the relatively unexplored massive regime. To see how the correlation function can be measured, it is helpful to define the fields $\hat{\Psi}_{x, \pm}=\left(\hat{\Psi}_{\uparrow} \pm \hat{\Psi}_{\downarrow}\right) / \sqrt{2}$ and $\hat{\Psi}_{y, \pm}=\left(\hat{\Psi}_{\uparrow} \mp i \hat{\Psi}_{\downarrow}\right) / \sqrt{2}$. When written in terms of these fields, the original correlation function becomes a combination of various density-density correlation functions involving the densities of $x$ and $y$, which can be straightforwardly measured. The detection scheme is depicted in figure 31(b). The waveplates mix the $\uparrow$ and $\downarrow$ components into $x$ and $y$ fields, one of which is then detected by using a polarization beam splitter in each arm. In this way, one could probe the scaling behaviour of the correlation functions and moreover infer the renormalization of mass due to the interaction.

\section{G. Experimental progress}

There are largely two promising experimental techniques to produce the waveguide-atom interface considered in this section. One is loading atoms in a hollowcore photonic band gap fibers (HCPBFs) and the other is coupling atoms to a tapered-fiber or more recently a two-dimensional photonic crystal. The purpose of this subsection is to discuss briefly the experimental progress in these fields.

The $\mathrm{HCPBF}$ is a waveguide which has a hollow-core surrounded by a photonic crystal structure [211, 212] and can therefore accommodate atoms in its core, where the guided mode resides. Strong confinement of light at the core then facilitates the sought-after strong atom-light interaction. The first experimental effort used pressure difference to pump hydrogen atoms into a HCPBF and thereby demonstrate stimulated Raman scattering [213]. It was soon followed by the generation of optical solitons in a xenon-filled fiber 214. A step towards strong lightmatter interaction has been made by demonstrating EIT using acetylene molecules [215] and rubidium molecules 216]. Optical guiding of thermal [217] and cold atoms 218 through a HCPBF and trapping of ultracold atoms in a HCPBF 219] have also been achieved. Most importantly for the subject of this review, guiding and trapping of laser cooled atoms was achieved using a magnetic guide [220]. In the same experiment, authors demonstrated all-optical switching using the four-level giant cross-Kerr effect introduced in Sect. IVA 1 with a moderate value of optical depth $\approx 30$. More recently, the use of hollowoptical-beam has allowed more efficient guiding of atoms, leading to an improved optical depth of 180 221.

The second promising system consists of atoms coupled to the guided mode of a tapered fiber. When an optical fiber is drawn out such that its diameter is smaller than the wavelength of the guided light [222], light mostly resides outside the fiber in the form of an evanescent wave, which enables easy coupling between the guided light and surrounding atoms. Coupling trapped cold atoms to the guided mode of a tapered fiber has been demonstrated [223, 224] and a hot vapor of atoms has been used to demonstrate EIT with a very low pump power [225]. Trapping and interrogation of cold atoms on the surface of a tapered-fiber by creating an optical lattice above the nanofiber surface has been achieved with an optical 
depth as high as $\approx 30$ [226, 227]. Most recently, stateinsensitive trapping of atoms has been achieved with an optical depth of 66 .

More recently, people have started looking at twodimensional nanophotonic structures involving photoniccrystal patterns [228, 229]. These types of systems allow strong light-atom coupling and may lead to an alternative platform to realize the models reviewed in this section. Moreover, other types of models can be readily simulated in this setup, such as the conventional cavityQED physics 230] and tunable many-body spin models [231]. As such, the platform is also a good candidate for a quantum information processor.

\section{H. Outlook: Rydberg polaritons}

Before we close the section, we would like to briefly mention another interesting paradigm to realize strong photon-photon interaction that has attracted much interest recently-Rydberg EIT. In Rydberg EIT, the strong long-range Rydberg interaction between atoms are converted to an effective photon-photon interaction through the appropriate use of EIT: one chooses the third state $(|c\rangle)$ of the atom to be a Rydberg state (typically requiring the level scheme to be of the ladder type). Due to an energy shift induced by the interaction between Rydberg states, the presence of a single photon in the system then creates a range (called the blockade radius) around the excitation where light propagation is forbidden. This means that two Rydberg excitations cannot travel within the blockade distance, or in other words they repel each other. The nonlinear interaction not only allows one to build photonic quantum information processors [232236, but also to investigate many-body physics in such systems. For example, there has been a proposal to create a BEC of stationary-light dark state Rydberg polaritons [203], as well as to observe Wigner Crystallization of photons in cold Rydberg ensembles [237]. Experimental progress in the field has been equally amazing; there have been demonstrations of EIT in a Rydberg medium 238, a strong Rydberg blockade [239]242, and even a microwave-controlled polariton-polariton interaction [243]. This rapidly growing field would not only provide a means to achieve strong photon-photon interaction but also a chance to simulate different types of phenomena by utilizing the available long-range interaction.

\section{SUMMARY AND OUTLOOK}

In this review, we have summarized numerous theoretical proposals and a few experimental demonstrations of strongly-interacting many-body photonic systems. We have started with the early proposals that realized that atom-induced photon-photon interactions used in tandem with coupled resonator structures can give rise to
Hubbard-like models often studied in condensed matter physics and cold atoms in optical lattices.

Section II reviewed the properties of the many-body ground states of closed coupled-resonator arrays as well as the possible equilibrium phases as calculated via meanfield approaches. We also presented proposals to simulate quantum spin-models and fractional quantum Hall states with photons.

Section III focused on works that go beyond equilibrium approaches, exploring experimentally valid situations where the system is naturally out of equilibrium. The differences and similarities between the driven-dissipative photonic Bose-Hubbard and JaynesCummings-Hubbard systems were first highlighted, followed by reviews on early investigations on interactiondriven fermionization and crystallization of photons in different scenarios. We also discussed two 'quenching scenarios', where transient effects of an initially prepared state are probed to reveal the underlying eigenstates of the system in an open but not driven system. Apart from observing the signatures of Mott-superfluid transition, an interesting 'self-trapping' was shown to occur, which has been verified experimentally in a superconducting circuit setup. We then summarized a couple of proposals to implement more exotic models. The first is an extended Hubbard model with the cross-Kerr interaction between nearest neighbours which, within the mean-field description, was shown to exhibit a supersolid-like phase. The second is a bosonic Kitaev chain with localized Majorana like modes near the end cavities. Such proposals reveal the versatility of CRA simulators. The section ends with a brief overview of interesting works that we could not cover in detail as well as a short survey on possible experimental platforms.

Section IV presented a very different paradigm which is more suitable for simulating continuum 1D quantum models, but nevertheless shares the main theme in that the central players are interacting photons. The system consists of an ensemble of 3- or 4-level atoms coupled to a 1D waveguide, with EIT or EIT-like coupling schemes. We showed how stationary states of light are formed, whose dynamics can be engineered to a large degree. This way, one can realize a photonic Tonks-Girardeau gas and furthermore add an effective potential whose depth can be varied to allow tuning between the Bose-Hubbard and quantum sine-Gordon limit, which in turn allows the 'pinning transition' to occur. We then reviewed a proposal to achieve the Bose-Einstein condensation of darkstate stationary-polaritons if the $2 \mathrm{D}$ nature of the crosssection of the waveguide becomes important and interaction is sufficiently strong. Next we discussed a generalization of the 1D system involving two species of atoms or two polarizations for the input pulses, which allows simulations of Luttinger liquids physics, spin-charge separation and BEC-BCS-like crossover. We then showed that one can also implement an interacting relativistic theory, namely the Thirring model, by engineering the dispersion relations of the propagating pulses of light and exploiting 
the strong EIT-based interactions. Strong interactions between intra-species photons enforce an effective Pauli principle in this $1 \mathrm{D}$ system, so that a fermionic model can be implemented in the photonic system. The section finishes with a mini survey on experimental progresses in doing EIT physics with strongly-coupled atom-waveguide interface and a more recent promising platform in this direction using Rydberg-EIT .

Hopefully we have convinced the reader that there have been many exciting-and still ongoing-developments in this young field. Although tremendous progress has been made in the last decade, there is still plenty of room for development. Experimentally, a large-size coupled resonator array with strong atom-resonator coupling in each site has not yet been fabricated, although impressive technological developments in circuit-QED indicate that we are very close to seeing one. Further developments in tools to probe and measure the non-equilibrium many-body states are necessary both experimentally and theoretically. Current theoretical tools are not sufficient to study even a moderate-sized driven-dissipative $2 \mathrm{D}$ arrays as we have discussed already, and developing a good numerical or analytical tool is of fundamental importance for further progress. One can ask many questions such as: Will developments in the tensor-networks help? Can we apply techniques from other area such as the Keldysh formalism to analytically find meaningful quantities? What are the optimal observables and ways to characterize the out-of-equilibrium phases in driven-dissipative photonic systems?

Regarding simulations of field theories using photonic systems, one of the key questions is whether the EITbased platform can be generalized to higher dimensions or a different platform is needed. A straightforward option is to utilize the two-dimensions within the waveguide (as we have seen in the BEC of dark state polaritons), but one difficulty is in maintaining a large optical depth while doing so. One can think of increasing the atom-photon coupling strength for this purpose or utilizing the strong interactions between the Rydberg atoms. The latter looks to be a promising avenue with exciting prospects for further discoveries.

\section{ACKNOWLEDGEMENTS}

We would like to acknowledge the financial support provided by the National Research Foundation and Ministry of Education Singapore (partly through the Tier 3 Grant "Random numbers from quantum processes"), and travel support by the EU IP-SIQS. We would like to thank the hospitality of the KITP during the program "Many-body physics with light"
[1] J Ignacio Cirac and Peter Zoller. Goals and opportunities in quantum simulation. Nature Physics, 8(4):264266, 2012.

[2] P. Hauke, F. M. Cucchietti, L. Tagliacozzo, I. Deutsch, and M. Lewenstein. Can one trust quantum simulators? Reports on Progress in Physics, 75(8):082401, August 2012.

[3] R. Blatt and C. F. Roos. Quantum simulations with trapped ions. Nat Phys, 8(4):277-284, 042012.

[4] Immanuel Bloch, Jean Dalibard, and Wilhelm Zwerger. Many-body physics with ultracold gases. Rev. Mod. Phys., 80:885-964, Jul 2008.

[5] D. G. Angelakis, M. F. Santos, and S. Bose. Photonblockade-induced Mott transitions and XY spin models in coupled cavity arrays. Physical Review A, 76(3):031805, September 2007.

[6] Michael J. Hartmann, Fernando G. S. L. Brandao, and Martin B. Plenio. Strongly interacting polaritons in coupled arrays of cavities. Nat Phys, 2(12):849-855, 12 2006.

[7] A. D. Greentree, C. Tahan, J. H. Cole, and L. C. L. Hollenberg. Quantum phase transitions of light. Nature Physics, 2:856-861, December 2006.

[8] D. Rossini and R. Fazio. Mott-Insulating and Glassy Phases of Polaritons in 1D Arrays of Coupled Cavities. Physical Review Letters, 99(18):186401, November 2007.

[9] Y. C. N. Na, S. Utsunomiya, and Y. Yamamoto. Strongly Correlated Photons in a Photonic Crystal. ArXiv Physics e-prints, February 2007.

[10] S. Bose, D. Angelakis, and D. Burgarth. Transfer of a polaritonic qubit through a coupled cavity array. Journal of Modern Optics, 54:2307-2314, September 2007.

[11] F. M. Hu, L. Zhou, T. Shi, and C. P. Sun. Coupled cavity QED for coherent control of photon transmission: Green-function approach for hybrid systems with two-level doping. Physical Review A, 76(1):013819, July 2007.

[12] R Chakrabarti and G Sreekumari. Propagation of single-excitation quantum states through jaynescummings-hubbard arrays. Journal of Physics B: Atomic, Molecular and Optical Physics, 44(11):115505, 2011.

[13] M. I. Makin, J. H. Cole, C. D. Hill, A. D. Greentree, and L. C. L. Hollenberg. Time evolution of the onedimensional Jaynes-Cummings-Hubbard Hamiltonian. Physical Review A, 80(4):043842, October 2009.

[14] M. Knap, E. Arrigoni, and W. von der Linden. Quantum phase transition and excitations of the Tavis-Cummings lattice model. Physical Review B, 82(4):045126, July 2010.

[15] M. J. Hartmann, F. G. S. L. Brandão, and M. B. Plenio. Effective Spin Systems in Coupled Microcavities. Physical Review Letters, 99(16):160501, October 2007.

[16] Dimitris G Angelakis and Alastair Kay. Weaving lightmatter qubits into a one way quantum computer. New Journal of Physics, 10(2):023012, 2008.

[17] Jaeyoon Cho, Dimitris G. Angelakis, and Sougato Bose. Heralded generation of entanglement with coupled cavities. Physical Review A, 78(2):022323-, 082008.

[18] J. Cho, D. G. Angelakis, and S. Bose. Fractional Quan- 
tum Hall State in Coupled Cavities. Physical Review Letters, 101(24):246809, December 2008.

[19] L. Lamata, J. León, T. Schätz, and E. Solano. Dirac equation and quantum relativistic effects in a single trapped ion. Phys. Rev. Lett., 98:253005, Jun 2007.

[20] J. Y. Vaishnav and Charles W. Clark. Observing zitterbewegung with ultracold atoms. Phys. Rev. Lett., 100:153002, Apr 2008.

[21] Gediminas Juzeliūnas, Julius Ruseckas, Markus Lindberg, Luis Santos, and Patrik Öhberg. Quasirelativistic behavior of cold atoms in light fields. Phys. Rev. A, 77:011802, Jan 2008.

[22] J. Otterbach, R. G. Unanyan, and M. Fleischhauer. Confining stationary light: Dirac dynamics and klein tunneling. Physical Review Letters, 102(6):063602-, 02 2009.

[23] S. Longhi. Klein tunneling in binary photonic superlattices. Phys. Rev. B, 81:075102, Feb 2010.

[24] J. Casanova, J. J. García-Ripoll, R. Gerritsma, C. F. Roos, and E. Solano. Klein tunneling and dirac potentials in trapped ions. Phys. Rev. A, 82:020101, Aug 2010.

[25] C Noh, B M Rodríguez-Lara, and D G Angelakis. Quantum simulation of neutrino oscillations with trapped ions. New Journal of Physics, 14(3):033028, 2012.

[26] Dimitris G. Angelakis, P. Das, and C. Noh. Probing the topological properties of the jackiw-rebbi model with light. Sci. Rep., 4, 082014.

[27] R. Gerritsma, G. Kirchmair, F. Zahringer, E. Solano, R. Blatt, and C. F. Roos. Quantum simulation of the dirac equation. Nature, 463(7277):68-71, 012010.

[28] R. Gerritsma, B. P. Lanyon, G. Kirchmair, F. Zähringer, C. Hempel, J. Casanova, J. J. García-Ripoll, E. Solano, R. Blatt, and C. F. Roos. Quantum simulation of the klein paradox with trapped ions. Phys. Rev. Lett., 106:060503, Feb 2011.

[29] Felix Dreisow, Matthias Heinrich, Robert Keil, Andreas Tünnermann, Stefan Nolte, Stefano Longhi, and Alexander Szameit. Classical simulation of relativistic zitterbewegung in photonic lattices. Phys. Rev. Lett., 105:143902, Sep 2010.

[30] F. Dreisow, R. Keil, A. Tünnermann, S. Nolte, S. Longhi, and A. Szameit. Klein tunneling of light in waveguide superlattices. EPL (Europhysics Letters), 97(1):10008, 2012.

[31] M. X. Huo, Y. Li, Z. Song, and C. P. Sun. Atomic entanglement versus visibility of photon interference for quantum criticality of a hybrid system. Physical Review A, 77(2):022103, February 2008.

[32] J. Cho, D. G. Angelakis, and S. Bose. Heralded generation of entanglement with coupled cavities. Physical Review A, 78(2):022323, August 2008.

[33] E. K. Irish. Ground-state entanglement in a coupledcavity model. Physical Review A, 80(4):043825, October 2009.

[34] S. M. Giampaolo and F. Illuminati. Long-distance entanglement in many-body atomic and optical systems. New Journal of Physics, 12(2):025019, February 2010.

[35] M. Aichhorn, M. Hohenadler, C. Tahan, and P. B. Littlewood. Quantum Fluctuations, Temperature, and Detuning Effects in Solid-Light Systems. Physical Review Letters, 100(21):216401, May 2008.

[36] J. Zhao, A. W. Sandvik, and K. Ueda. Insulator to superfluid transition in coupled photonic cavities in two dimensions. ArXiv e-prints, June 2008.

[37] A. Mering, M. Fleischhauer, P. A. Ivanov, and K. Singer. Analytic approximations to the phase diagram of the Jaynes-Cummings-Hubbard model. Physical Review A, 80(5):053821, November 2009.

[38] S. Schmidt and G. Blatter. Excitations of Strongly Correlated Lattice Polaritons. Physical Review Letters, 104(21):216402, May 2010.

[39] M. Knap, E. Arrigoni, and W. von der Linden. Spectral properties of coupled cavity arrays in one dimension. Physical Review B, 81(10):104303, March 2010.

[40] M. I. Makin, J. H. Cole, C. Tahan, L. C. L. Hollenberg, and A. D. Greentree. Quantum phase transitions in photonic cavities with two-level systems. Physical Review A, 77(5):053819, May 2008.

[41] Z.-X. Chen, Z.-W. Zhou, X. Zhou, X.-F. Zhou, and G.C. Guo. Quantum simulation of Heisenberg spin chains with next-nearest-neighbor interactions in coupled cavities. Physical Review A, 81(2):022303, February 2010.

[42] M. J. Hartmann, F. G. S. L. Brandão, and M. B. Plenio. A polaritonic two-component Bose-Hubbard model. New Journal of Physics, 10(3):033011, March 2008.

[43] M. Paternostro, G. S. Agarwal, and M. S. Kim. Solitonic behaviour in coupled multi atom-cavity systems. New Journal of Physics, 11(1):013059, January 2009.

[44] S. Schmidt and G. Blatter. Strong Coupling Theory for the Jaynes-Cummings-Hubbard Model. Physical Review Letters, 103(8):086403, August 2009.

[45] E. S. Kyoseva, D. G. Angelakis, and L. C. Kwek. A single-interaction step implementation of a quantum search in coupled micro-cavities. EPL (Europhysics Letters), 89:20005, January 2010.

[46] A. A. Houck, H. E. Türeci, and J. Koch. On-chip quantum simulation with superconducting circuits. Nature Physics, 8:292-299, April 2012.

[47] A. Majumdar, A. Rundquist, M. Bajcsy, V. D. Dasika, S. R. Bank, and J. Vučković. Design and analysis of photonic crystal coupled cavity arrays for quantum simulation. Physical Review B, 86(19):195312, November 2012.

[48] G Lepert, M Trupke, M J Hartmann, M B Plenio, and E A Hinds. Arrays of waveguide-coupled optical cavities that interact strongly with atoms. New Journal of Physics, 13(11):113002, 2011.

[49] D. E. Chang, V. Gritsev, G. Morigi, V. Vuletić, M. D. Lukin, and E. A. Demler. Crystallization of strongly interacting photons in a nonlinear optical fibre. Nature Physics, 4:884-889, November 2008.

[50] D. G. Angelakis, M. Huo, E. Kyoseva, and L. C. Kwek. Luttinger Liquid of Photons and Spin-Charge Separation in Hollow-Core Fibers. Physical Review Letters, 106(15):153601, April 2011.

[51] Dimitris G. Angelakis, Ming-Xia Huo, Darrick Chang, Leong Chuan Kwek, and Vladimir Korepin. Mimicking interacting relativistic theories with stationary pulses of light. Phys. Rev. Lett., 110:100502, Mar 2013.

[52] Iacopo Carusotto and Cristiano Ciuti. Quantum fluids of light. Rev. Mod. Phys., 85:299-366, Feb 2013.

[53] I. Carusotto, T. Volz, and A. Imamoğlu. Feshbach blockade: Single-photon nonlinear optics using resonantly enhanced cavity polariton scattering from biexciton states. EPL (Europhysics Letters), 90(3):37001, 2010 . 
[54] J. Kasprzak, M. Richard, S. Kundermann, A. Baas, P. Jeambrun, J. M. J. Keeling, F. M. Marchetti, M. H. Szymanska, R. Andre, J. L. Staehli, V. Savona, P. B. Littlewood, B. Deveaud, and Le Si Dang. Boseeinstein condensation of exciton polaritons. Nature, 443(7110):409-414, 092006.

[55] Y. Lai and H. A. Haus. Quantum theory of solitons in optical fibers. i. time-dependent hartree approximation. Physical Review A, 40(2):844-853, 071989.

[56] Y. Lai and H. A. Haus. Quantum theory of solitons in optical fibers. ii. exact solution. Physical Review A, 40(2):854-866, 071989.

[57] Pierre-Élie Larré and Iacopo Carusotto. Propagation of a quantum fluid of light in a cavityless nonlinear optical medium: General theory and response to quantum quenches. Physical Review A, 92(4):043802-, 102015.

[58] Amnon Yariv, Yong Xu, Reginald K. Lee, and Axel Scherer. Coupled-resonator optical waveguide: a proposal and analysis. Opt. Lett., 24(11):711-713, Jun 1999.

[59] Mehmet Bayindir, B. Temelkuran, and E. Ozbay. Tightbinding description of the coupled defect modes in threedimensional photonic crystals. Physical Review Letters, 84(10):2140-2143, 032000.

[60] George Paloczi, Yanyi Huang, Amnon Yariv, and Shayan Mookherjea. Polymeric mach-zehnder interferometer using serially coupled microring resonators. $O p$ tics Express, 11(21):2666-2671, 2003.

[61] M. H. Shih, W. J. Kim, Wan Kuang, J. R. Cao, H. Yukawa, S. J. Choi, J. D. O'Brien, P. D. Dapkus, and W. K. Marshall. Two-dimensional photonic crystal mach-zehnder interferometers. Applied Physics Letters, 84(4):460-462, 2004.

[62] Hatice Altug and Jelena Vučković. Two-dimensional coupled photonic crystal resonator arrays. Applied Physics Letters, 84(2):161-163, 2004.

[63] Antonio Badolato, Kevin Hennessy, Mete Atatüre, Jan Dreiser, Evelyn Hu, Pierre M. Petroff, and Atac Imamoğlu. Deterministic coupling of single quantum dots to single nanocavity modes. Science, 308(5725):1158-1161, 2005.

[64] A. Wallraff, D. I. Schuster, A. Blais, L. Frunzio, R. S. Huang, J. Majer, S. Kumar, S. M. Girvin, and R. J. Schoelkopf. Strong coupling of a single photon to a superconducting qubit using circuit quantum electrodynamics. Nature, 431(7005):162-167, 092004.

[65] Dimitris G. Angelakis, Marcelo F. Santos, Vassilios Yannopapas, and Artur Ekert. A proposal for the implementation of quantum gates with photonic-crystal waveguides. Physics Letters A, 362(5-6):377-380, 3 2007.

[66] A. Imamoḡlu, H. Schmidt, G. Woods, and M. Deutsch. Strongly interacting photons in a nonlinear cavity. Physical Review Letters, 79(8):1467-1470, 081997.

[67] K. M. Birnbaum, A. Boca, R. Miller, A. D. Boozer, T. E. Northup, and H. J. Kimble. Photon blockade in an optical cavity with one trapped atom. Nature, 436(7047):87-90, 072005.

[68] L.-M. Duan, E. Demler, and M. D. Lukin. Controlling spin exchange interactions of ultracold atoms in optical lattices. Phys. Rev. Lett., 91:090402, Aug 2003.

[69] M.J. Hartmann, F.G.S.L. Brandão, and M.B. Plenio. Quantum many-body phenomena in coupled cavity ar- rays. Laser and Photonics Reviews, 2(6):527-556, 2008.

[70] M. J. Hartmann and M. B. Plenio. Strong Photon Nonlinearities and Photonic Mott Insulators. Physical Review Letters, 99(10):103601, September 2007.

[71] D. Jaksch, C. Bruder, J. I. Cirac, C. W. Gardiner, and P. Zoller. Cold bosonic atoms in optical lattices. Physical Review Letters, 81(15):3108-3111, 101998.

[72] P. Pippan, H. G. Evertz, and M. Hohenadler. Excitation spectra of strongly correlated lattice bosons and polaritons. Physical Review A, 80(3):033612, September 2009.

[73] M. Hohenadler, M. Aichhorn, S. Schmidt, and L. Pollet. Dynamical critical exponent of the Jaynes-CummingsHubbard model. Physical Review A, 84(4):041608, October 2011.

[74] M. Hohenadler, M. Aichhorn, L. Pollet, and S. Schmidt. Polariton Mott insulator with trapped ions or circuit QED. Physical Review A, 85(1):013810, January 2012.

[75] Michael A. Nielsen. Cluster-state quantum computation. Reports on Mathematical Physics, 57(1):147-161, 22006.

[76] P. Li, Q. Gong, and G. Guo. Effective generation of Ising interaction and cluster states in coupled microcavities. ArXiv e-prints, December 2007.

[77] P. B. Li, Y. Gu, Q. H. Gong, and G. C. Guo. Generation of Ising interaction and cluster states in a onedimensional coupled resonator waveguide. European Physical Journal D, 55:205-209, October 2009.

[78] A. Kay and D. G. Angelakis. Reproducing spin lattice models in strongly coupled atom-cavity systems. EPL (Europhysics Letters), 84(2):20001, 2008.

[79] D. Burgarth. Quantum State Transfer with Spin Chains. ArXiv e-prints, April 2007.

[80] Sujit Sarkar. Quantum field theoretical study of an effective spin model in coupled optical cavity arrays. Physica B: Condensed Matter, 407(1):44-48, 12012.

[81] C. Bruder, Rosario Fazio, and Gerd Schön. Superconductor-mott-insulator transition in bose systems with finite-range interactions. Physical Review B, 47(1):342-347, 011993.

[82] Daniel Loss and David P. DiVincenzo. Quantum computation with quantum dots. Physical Review A, 57(1):120-126, 011998.

[83] J. J. García-Ripoll, M. A. Martin-Delgado, and J. I. Cirac. Implementation of spin hamiltonians in optical lattices. Physical Review Letters, 93(25):250405-, 12 2004.

[84] J. Cho, D. G. Angelakis, and S. Bose. Simulation of high-spin Heisenberg models in coupled cavities. Physical Review A, 78(6):062338, December 2008.

[85] D. C. Tsui, H. L. Stormer, and A. C. Gossard. Twodimensional magnetotransport in the extreme quantum limit. Physical Review Letters, 48(22):1559-1562, 05 1982.

[86] Maciej Lewenstein, Anna Sanpera, Veronica Ahufinger, Bogdan Damski, Aditi Sen(De), and Ujjwal Sen. Ultracold atomic gases in optical lattices: mimicking condensed matter physics and beyond. Advances in Physics, 56(2):243-379, 2014/02/25 2007.

[87] N. K. Wilkin and J. M. F. Gunn. Condensation of "composite bosons" in a rotating bec. Physical Review Letters, 84(1):6-9, 012000.

[88] G. Juzeliūnas and P. Öhberg. Slow light in degenerate 
fermi gases. Physical Review Letters, 93(3):033602-, 07 2004.

[89] D Jaksch and P Zoller. Creation of effective magnetic fields in optical lattices: the hofstadter butterfly for cold neutral atoms. New Journal of Physics, 5(1):56, 2003.

[90] Anders S. Sørensen, Eugene Demler, and Mikhail D. Lukin. Fractional quantum hall states of atoms in optical lattices. Physical Review Letters, 94(8):086803-, 03 2005.

[91] Jens Koch, Andrew A. Houck, Karyn Le Hur, and S. M. Girvin. Time-reversal-symmetry breaking in circuitqed-based photon lattices. Phys. Rev. A, 82:043811, Oct 2010.

[92] A. Nunnenkamp, J. Koch, and S. M. Girvin. Synthetic gauge fields and homodyne transmission in Jaynes-Cummings lattices. New Journal of Physics, 13(9):095008, September 2011.

[93] R. O. Umucalılar and I. Carusotto. Artificial gauge field for photons in coupled cavity arrays. Phys. Rev. A, 84:043804, Oct 2011.

[94] Mohammad Hafezi, Eugene A. Demler, Mikhail D. Lukin, and Jacob M. Taylor. Robust optical delay lines with topological protection. Nat Phys, 7(11):907-912, 112011.

[95] Hafezi M., Mittal S., Fan J., Migdall A., and Taylor J. M. Imaging topological edge states in silicon photonics. Nat Photon, 7(12):1001-1005, 122013.

[96] R. O. Umucalılar and I. Carusotto. Fractional quantum hall states of photons in an array of dissipative coupled cavities. Phys. Rev. Lett., 108:206809, May 2012.

[97] A. L. C. Hayward, A. M. Martin, and A. D. Greentree. Fractional Quantum Hall Physics in JaynesCummings-Hubbard Lattices. Physical Review Letters, 108(22):223602, June 2012.

[98] Mohammad Hafezi, Mikhail D. Lukin, and Jacob M. Taylor. Non-equilibrium fractional quantum hall state of light. New Journal of Physics, 15(6):063001, 2013.

[99] Changsuk Noh and Dimitris G. Angelakis. Simulating topological effects with photons in coupled qed cavity arrays. International Journal of Modern Physics B, 28(02):1441003, 2014.

[100] F. D. M. Haldane and E. H. Rezayi. Periodic laughlinjastrow wave functions for the fractional quantized hall effect. Physical Review B, 31(4):2529-2531, 021985.

[101] Kristian Baumann, Christine Guerlin, Ferdinand Brennecke, and Tilman Esslinger. Dicke quantum phase transition with a superfluid gas in an optical cavity. $\mathrm{Na}$ ture, 464(7293):1301-1306, 042010.

[102] Jan Klaers, Julian Schmitt, Frank Vewinger, and Martin Weitz. Bose-einstein condensation of photons in an optical microcavity. Nature, 468(7323):545-548, 112010.

[103] A. Chiocchetta, A. Gambassi, and I. Carusotto. Laser operation and Bose-Einstein condensation: analogies and differences. ArXiv e-prints, March 2015.

[104] A. Tomadin and R. Fazio. Many-body phenomena in qed-cavity arrays [invited]. Journal of the Optical Society of America B, 27(6):A130-A136, 2010.

[105] S. Schmidt and J. Koch. Circuit QED lattices: Towards quantum simulation with superconducting circuits. Annalen der Physik, 525:395-412, 2013.

[106] L. M. Sieberer, S. D. Huber, E. Altman, and S. Diehl. Dynamical critical phenomena in driven-dissipative systems. Physical Review Letters, 110(19):195301-, 05 2013.
[107] Markus Müller, Sebastian Diehl, Guido Pupillo, and Peter Zoller. Engineered Open Systems and Quantum Simulations with Atoms and Ions, volume Volume 61, pages 1-80. Academic Press, 2012.

[108] Sebastian Diehl, Andrea Tomadin, Andrea Micheli, Rosario Fazio, and Peter Zoller. Dynamical phase transitions and instabilities in open atomic many-body systems. Physical Review Letters, 105(1):015702-, 072010.

[109] Tomaž Prosen and Iztok Pižorn. Quantum phase transition in a far-from-equilibrium steady state of an $\$ \mathrm{xy} \$$ spin chain. Physical Review Letters, 101(10):105701-, 092008.

[110] Birger Horstmann, J. Ignacio Cirac, and Géza Giedke. Noise-driven dynamics and phase transitions in fermionic systems. Physical Review A, 87(1):012108, 012013.

[111] M. Leib, F. Deppe, A. Marx, R. Gross, and M. J. Hartmann. Networks of nonlinear superconducting transmission line resonators. New Journal of Physics, 14(7):075024, July 2012.

[112] T Grujic, S R Clark, D Jaksch, and D G Angelakis. Nonequilibrium many-body effects in driven nonlinear resonator arrays. New Journal of Physics, 14(10):103025, 2012.

[113] Howard J. Carmichael. Statistical Methods in Quantum Optics 1, volume 1. Springer-Verlag Berlin Heidelberg, 2002.

[114] I. Carusotto, D. Gerace, H. E. Tureci, S. de Liberato, C. Ciuti, and A. Imamoğlu. Fermionized Photons in an Array of Driven Dissipative Nonlinear Cavities. Physical Review Letters, 103(3):033601, July 2009.

[115] M. J. Hartmann. Polariton Crystallization in Driven Arrays of Lossy Nonlinear Resonators. Physical Review Letters, 104(11):113601, March 2010.

[116] M. Girardeau. Relationship between systems of impenetrable bosons and fermions in one dimension. Journal of Mathematical Physics, 1(6):516-523, 1960.

[117] M. Knap, E. Arrigoni, W. von der Linden, and J. H. Cole. Emission characteristics of laser-driven dissipative coupled-cavity systems. Physical Review A, 83(2):023821, February 2011.

[118] F. Verstraete, V. Murg, and J. I. Cirac. Matrix product states, projected entangled pair states, and variational renormalization group methods for quantum spin systems. Advances in Physics, 57(2):143-224, 2014/10/07 2008.

[119] Ulrich Schollwöck. The density-matrix renormalization group in the age of matrix product states. Annals of Physics, 326(1):96-192, 12011.

[120] Elena del Valle and Michael J Hartmann. Correlator expansion approach to stationary states of weakly coupled cavity arrays. Journal of Physics B: Atomic, Molecular and Optical Physics, 46(22):224023, 2013.

[121] Peter Degenfeld-Schonburg and Michael J. Hartmann. Self-consistent projection operator theory for quantum many-body systems. Phys. Rev. B, 89:245108, Jun 2014.

[122] A. Tomadin, V. Giovannetti, R. Fazio, D. Gerace, I. Carusotto, H. E. Türeci, and A. Imamoglu. Signatures of the superfluid-insulator phase transition in laser-driven dissipative nonlinear cavity arrays. Physical Review A, 81(6):061801, June 2010.

[123] T. Hümmer, G. M. Reuther, P. Hänggi, and D. Zueco. Nonequilibrium phases in hybrid arrays with flux qubits and nitrogen-vacancy centers. Physical Review A, 
85(5):052320, May 2012.

[124] S. Schmidt, D. Gerace, A. A. Houck, G. Blatter, and H. E. Türeci. Nonequilibrium delocalization-localization transition of photons in circuit quantum electrodynamics. Physical Review B, 82(10):100507, September 2010.

[125] J. Raftery, D. Sadri, S. Schmidt, H. E. Türeci, and A. A. Houck. Observation of a dissipation-induced classical to quantum transition. Phys. Rev. X, 4:031043, Sep 2014.

[126] S. M. Jensen. The nonlinear coherent coupler. Quantum Electronics, IEEE Journal of, 18(10):1580-1583, Oct 1982.

[127] J. C. Eilbeck, P. S. Lomdahl, and A. C. Scott. The discrete self-trapping equation. Physica D: Nonlinear Phenomena, 16(3):318-338, 71985.

[128] A. Smerzi, S. Fantoni, S. Giovanazzi, and S. R. Shenoy. Quantum coherent atomic tunneling between two trapped bose-einstein condensates. Physical Review Letters, 79(25):4950-4953, 121997.

[129] Michael Albiez, Rudolf Gati, Jonas Fölling, Stefan Hunsmann, Matteo Cristiani, and Markus K. Oberthaler. Direct observation of tunneling and nonlinear selftrapping in a single bosonic josephson junction. Physical Review Letters, 95(1):010402-, 062005.

[130] S. Levy, E. Lahoud, I. Shomroni, and J. Steinhauer. The a.c. and d.c. josephson effects in a bose-einstein condensate. Nature, 449(7162):579-583, 102007.

[131] Davide Sarchi, Iacopo Carusotto, Michiel Wouters, and Vincenzo Savona. Coherent dynamics and parametric instabilities of microcavity polaritons in double-well systems. Physical Review B, 77(12):125324-, 032008.

[132] Nikolaos Schetakis, Thomas Grujic, Stephen Clark, Dieter Jaksch, and Dimitris Angelakis. Frozen photons in jaynes-cummings arrays. Journal of Physics B: Atomic, Molecular and Optical Physics, 46(22):224025, 2013.

[133] Jiasen Jin, Davide Rossini, Rosario Fazio, Martin Leib, and Michael J. Hartmann. Photon solid phases in driven arrays of nonlinearly coupled cavities. Physical Review Letters, 110(16):163605-, 042013.

[134] Jiasen Jin, Davide Rossini, Martin Leib, Michael J. Hartmann, and Rosario Fazio. Steady-state phase diagram of a driven qed-cavity array with cross-kerr nonlinearities. Phys. Rev. A, 90:023827, Aug 2014.

[135] B. Bujnowski, J. K. Corso, A. L. C. Hayward, J. H. Cole, and A. M. Martin. Supersolid phases of light in extended jaynes-cummings-hubbard systems. Physical Review A, 90(4):043801-, 102014.

[136] C.-E. Bardyn and A. İmamoğlu. Majorana-like Modes of Light in a One-Dimensional Array of Nonlinear Cavities. Physical Review Letters, 109(25):253606, December 2012.

[137] Chaitanya Joshi, Felix Nissen, and Jonathan Keeling. Quantum correlations in the one-dimensional driven dissipative \$xy\$ model. Physical Review A, 88(6):063835-, 122013.

[138] A Yu Kitaev. Unpaired majorana fermions in quantum wires. Physics-Uspekhi, 44(10S):131, 2001.

[139] Johan Nilsson, A. R. Akhmerov, and C. W. J. Beenakker. Splitting of a cooper pair by a pair of majorana bound states. Phys. Rev. Lett., 101:120403, Sep 2008.

[140] K. T. Law, Patrick A. Lee, and T. K. Ng. Majorana fermion induced resonant andreev reflection. Phys. Rev. Lett., 103:237001, Dec 2009.
[141] Karsten Flensberg. Non-abelian operations on majorana fermions via single-charge control. Phys. Rev. Lett., 106:090503, Mar 2011.

[142] D. Gerace, H. E. Türeci, A. Imamoglu, V. Giovannetti, and R. Fazio. The quantum-optical Josephson interferometer. Nature Physics, 5:281-284, April 2009.

[143] S. Ferretti, L. C. Andreani, H. E. Türeci, and D. Gerace. Photon correlations in a two-site nonlinear cavity system under coherent drive and dissipation. Physical Review A, 82(1):013841, July 2010.

[144] M. Leib and M. J. Hartmann. Bose-Hubbard dynamics of polaritons in a chain of circuit quantum electrodynamics cavities. New Journal of Physics, 12(9):093031, September 2010.

[145] Alberto Biella, Leonardo Mazza, Iacopo Carusotto, Davide Rossini, and Rosario Fazio. Photon transport in a dissipative chain of nonlinear cavities. Physical Review A, 91(5):053815-, 052015.

[146] Changhyoup Lee, Changsuk Noh, Nikolaos Schetakis, and Dimitris G. Angelakis. Few-photon transport in many-body photonic systems: A scattering approach. Physical Review A, 92(6):063817-, 122015.

[147] J. Ruiz-Rivas, E. del Valle, C. Gies, P. Gartner, and M. J. Hartmann. Spontaneous collective coherence in driven dissipative cavity arrays. Physical Review A, 90(3):033808-, 092014.

[148] Felix Nissen, Sebastian Schmidt, Matteo Biondi, Gianni Blatter, Hakan E. Türeci, and Jonathan Keeling. Nonequilibrium dynamics of coupled qubit-cavity arrays. Physical Review Letters, 108(23):233603-, 062012.

[149] T. Grujic, S. R. Clark, D. Jaksch, and D. G. Angelakis. Repulsively induced photon superbunching in driven resonator arrays. Physical Review A, 87(5):053846-, 05 2013.

[150] T. C. H. Liew and V. Savona. Single photons from coupled quantum modes. Physical Review Letters, 104(18):183601-, 052010.

[151] M. Bamba, A. Imamoğlu, I. Carusotto, and C. Ciuti. Origin of strong photon antibunching in weakly nonlinear photonic molecules. Physical Review A, 83(2):021802, February 2011.

[152] Dario Gerace and Vincenzo Savona. Unconventional photon blockade in doubly resonant microcavities with second-order nonlinearity. Physical Review A, 89(3):031803-, 032014.

[153] D. G. Angelakis, S. Bose, and S. Mancini. Steady-state entanglement between hybrid light-matter qubits. EPL (Europhysics Letters), 85(2):20007, 2009.

[154] D. G. Angelakis, L. Dai, and L. C. Kwek. Coherent control of long-distance steady-state entanglement in lossy resonator arrays. EPL (Europhysics Letters), 91(1):10003, 2010.

[155] Li Dai, Dimitris G. Angelakis, Leong Chuan Kwek, and S. Mancini. Correlations and thermalization in driven cavity arrays. AIP Conference Proceedings, 1384(1):168-176, 2011.

[156] T. C. H. Liew and V. Savona. Quantum entanglement in nanocavity arrays. Physical Review A, 85(5):050301, May 2012.

[157] T. C. H. Liew and V. Savona. Multimode entanglement in coupled cavity arrays. New Journal of Physics, 15(2):025015, February 2013.

[158] Alexandre Le Boité, Giuliano Orso, and Cristiano Ciuti. Steady-state phases and tunneling-induced instabilities 
in the driven dissipative bose-hubbard model. Physical Review Letters, 110(23):233601-, 062013.

[159] Alexandre Le Boité, Giuliano Orso, and Cristiano Ciuti. Bose-hubbard model: Relation between drivendissipative steady states and equilibrium quantum phases. Physical Review A, 90(6):063821-, 122014.

[160] J. J. Mendoza-Arenas, S. R. Clark, S. Felicetti, G. Romero, E. Solano, D. G. Angelakis, and D. Jaksch. Beyond mean-field bistability in driven-dissipative lattices: Bunching-antibunching transition and quantum simulation. Physical Review A, 93(2):023821-, 022016.

[161] S. Finazzi, A. Le Boité, F. Storme, A. Baksic, and C. Ciuti. Corner-space renormalization method for driven-dissipative two-dimensional correlated systems. Physical Review Letters, 115(8):080604-, 082015.

[162] Tomoki Ozawa and Iacopo Carusotto. Anomalous and quantum hall effects in lossy photonic lattices. Phys. Rev. Lett., 112:133902, Apr 2014.

[163] A. C. Berceanu, H. M. Price, Ozawa T., and I. Carusotto. Momentum-space landau levels in drivendissipative cavity arrays. arXiv:1510.03054, 2015.

[164] Mohammad Hafezi. Measuring topological invariants in photonic systems. Physical Review Letters, 112(21):210405-, 052014.

[165] C-E Bardyn, S D Huber, and O Zilberberg. Measuring topological invariants in small photonic lattices. New Journal of Physics, 16(12):123013, 2014.

[166] Eliot Kapit, Mohammad Hafezi, and Steven H. Simon. Induced self-stabilization in fractional quantum hall states of light. Physical Review X, 4(3):031039-, 09 2014.

[167] J. Lebreuilly, I. Carusotto, and M. Wouters. Strongly interacting photons in arrays of dissipative nonlinear cavities under a frequency-dependent incoherent pumping. arXiv:1502.04016, 2015.

[168] Kenji Toyoda, Yuta Matsuno, Atsushi Noguchi, Shinsuke Haze, and Shinji Urabe. Experimental realization of a quantum phase transition of polaritonic excitations. Phys. Rev. Lett., 111:160501, Oct 2013.

[169] V. G. Sala, D. D. Solnyshkov, I. Carusotto, T. Jacqmin, A. Lemaître, H. Terças, A. Nalitov, M. Abbarchi, E. Galopin, I. Sagnes, J. Bloch, G. Malpuech, and A. Amo. Spin-orbit coupling for photons and polaritons in microstructures. Physical Review X, 5(1):011034-, 03 2015.

[170] A. Majumdar, A. Rundquist, M. Bajcsy, and J. Vučković. Cavity quantum electrodynamics with a single quantum dot coupled to a photonic molecule. Physical Review B, 86(4):045315, July 2012.

[171] C. Eichler, Y. Salathe, J. Mlynek, S. Schmidt, and A. Wallraff. Quantum-limited amplification and entanglement in coupled nonlinear resonators. Physical Review Letters, 113(11):110502-, 092014.

[172] D. L. Underwood, W. E. Shanks, J. Koch, and A. A. Houck. Low-disorder microwave cavity lattices for quantum simulation with photons. Physical Review A, 86(2):023837, August 2012.

[173] D. L. Underwood, W. E. Shanks, A. C. Y. Li, L. Ateshian, J. Koch, and A. A. Houck. Imaging Photon Lattice States by Scanning Defect Microscopy. arXiv:1510.08428, 2015.

[174] S. E. Harris, J. E. Field, and A. Imamoğlu. Nonlinear optical processes using electromagnetically induced transparency. Physical Review Letters, 64(10):1107-
1110, 031990.

[175] Stephen E. Harris. Electromagnetically induced transparency. Physics Today, 50(7):36-42, 071997.

[176] Michael Fleischhauer, Atac Imamoglu, and Jonathan P. Marangos. Electromagnetically induced transparency: Optics in coherent media. Reviews of Modern Physics, 77(2):633-673, 072005.

[177] M. Bajcsy, A. S. Zibrov, and M. D. Lukin. Stationary pulses of light in an atomic medium. Nature, 426(6967):638-641, 122003.

[178] M. Fleischhauer and M. D. Lukin. Dark-state polaritons in electromagnetically induced transparency. Physical Review Letters, 84(22):5094-5097, 052000.

[179] F. E. Zimmer, A. André, M. D. Lukin, and M. Fleischhauer. Coherent control of stationary light pulses. Optics Communications, 264(2):441-453, 82006.

[180] A. André, M. Bajcsy, A. S. Zibrov, and M. D. Lukin. Nonlinear optics with stationary pulses of light. Physical Review Letters, 94(6):063902-, 022005.

[181] H. Schmidt and A. Imamoglu. Giant kerr nonlinearities obtained by electromagnetically induced transparency. Optics Letters, 21(23):1936-1938, 1996.

[182] M. Kiffner and M. J. Hartmann. Master equation approach for interacting slow- and stationary-light polaritons. Physical Review A, 82(3):033813, September 2010.

[183] Lewi Tonks. The complete equation of state of one, two and three-dimensional gases of hard elastic spheres. Phys. Rev., 50:955-963, Nov 1936.

[184] N. Syassen, D. M. Bauer, M. Lettner, T. Volz, D. Dietze, J. J. García-Ripoll, J. I. Cirac, G. Rempe, and S. Dürr. Strong dissipation inhibits losses and induces correlations in cold molecular gases. Science, 320(5881):13291331, 2008.

[185] M. Kiffner and M. J. Hartmann. Dissipation-induced tonks-girardeau gas of polaritons. Phys. Rev. A, 81:021806, Feb 2010.

[186] Thierry Giamarchi. Quantum Physics in One Dimension. Oxford University Press, 2003.

[187] Elliott H. Lieb and Werner Liniger. Exact analysis of an interacting bose gas. i. the general solution and the ground state. Physical Review, 130(4):1605-1616, 05 1963.

[188] Toshiya Kinoshita, Trevor Wenger, and David S. Weiss. Observation of a one-dimensional tonks-girardeau gas. Science, 305(5687):1125-1128, 082004.

[189] Belen Paredes, Artur Widera, Valentin Murg, Olaf Mandel, Simon Folling, Ignacio Cirac, Gora V. Shlyapnikov, Theodor W. Hansch, and Immanuel Bloch. Tonks-girardeau gas of ultracold atoms in an optical lattice. Nature, 429(6989):277-281, 052004.

[190] J. Friedel. Metallic alloys. Il Nuovo Cimento, 7(2):287311, 1958.

[191] A. Lenard. One-dimensional impenetrable bosons in thermal equilibrium. Journal of Mathematical Physics, 7(7):1268-1272, 071966.

[192] M.-X. Huo and D. G. Angelakis. Sine-Gordon and BoseHubbard dynamics with photons in a hollow-core fiber. Physical Review A, 85(2):023821, February 2012.

[193] Kristian Rymann Hansen and Klaus Mølmer. Trapping of light pulses in ensembles of stationary atoms. Physical Review A, 75(5):053802-, 052007.

[194] Gor Nikoghosyan and Michael Fleischhauer. Stationary light in cold-atomic gases. Physical Review A, 80(1):013818-, 072009. 
[195] H. P. Büchler, G. Blatter, and W. Zwerger. Commensurate-incommensurate transition of cold atoms in an optical lattice. Physical Review Letters, 90(13):130401-, 042003.

[196] Matthew P. A. Fisher, Peter B. Weichman, G. Grinstein, and Daniel S. Fisher. Boson localization and the superfluid-insulator transition. Physical Review B, 40(1):546-570, 071989.

[197] F. D. M. Haldane. Effective harmonic-fluid approach to low-energy properties of one-dimensional quantum fluids. Physical Review Letters, 47(25):1840-1843, 12 1981.

[198] Elmar Haller, Russell Hart, Manfred J. Mark, Johann G. Danzl, Lukas Reichsollner, Mattias Gustavsson, Marcello Dalmonte, Guido Pupillo, and HannsChristoph Nagerl. Pinning quantum phase transition for a luttinger liquid of strongly interacting bosons. $\mathrm{Na}$ ture, 466(7306):597-600, 072010.

[199] S. A. Moiseev and B. S. Ham. Quantum manipulation of two-color stationary light: Quantum wavelength conversion. Physical Review A, 73(3):033812-, 032006.

[200] F. E. Zimmer, J. Otterbach, R. G. Unanyan, B. W. Shore, and M. Fleischhauer. Dark-state polaritons for multicomponent and stationary light fields. Physical Review A, 77(6):063823-, 062008.

[201] Yen-Wei Lin, Wen-Te Liao, Thorsten Peters, HungChih Chou, Jian-Siung Wang, Hung-Wen Cho, PeiChen Kuan, and Ite A. Yu. Stationary light pulses in cold atomic media and without bragg gratings. Physical Review Letters, 102(21):213601-, 052009.

[202] Michael Fleischhauer, Johannes Otterbach, and Razmik G. Unanyan. Bose-einstein condensation of stationary-light polaritons. Physical Review Letters, 101(16):163601-, 102008.

[203] G. Nikoghosyan, F. E. Zimmer, and M. B. Plenio. Dipolar bose-einstein condensate of dark-state polaritons. Phys. Rev. A, 86:023854, Aug 2012.

[204] M.-X. Huo, D. G. Angelakis, and L. C. Kwek. Spinons and holons with polarized photons in a nonlinear waveguide. New Journal of Physics, 14(7):075027, July 2012.

[205] B. Paredes and J. I. Cirac. From cooper pairs to luttinger liquids with bosonic atoms in optical lattices. Physical Review Letters, 90(15):150402-, 042003.

[206] M.-X. Huo, C. Noh, B. M. Rodríguez-Lara, and D. G. Angelakis. Quantum simulation of Cooper pairing with photons. Physical Review A, 86(4):043840, October 2012.

[207] A. Kleine, C. Kollath, I. P. McCulloch, T. Giamarchi, and U. Schollwöck. Spin-charge separation in two-component bose gases. Physical Review A, 77(1):013607-, 012008.

[208] E. Orignac and T. Giamarchi. Vortices in coupled planes with columnar disorder and bosonic ladders. Physical Review B, 57(18):11713-11729, 051998.

[209] Walter E Thirring. A soluble relativistic field theory. Annals of Physics, 3(1):91-112, 11958.

[210] Sidney Coleman. Quantum sine-gordon equation as the massive thirring model. Physical Review D, 11(8):20882097, 041975.

[211] R. F. Cregan, B. J. Mangan, J. C. Knight, T. A. Birks, P. St. J. Russell, P. J. Roberts, and D. C. Allan. Singlemode photonic band gap guidance of light in air. Science, 285(5433):1537-1539, 091999.

[212] Charlene M. Smith, Natesan Venkataraman, Michael T.
Gallagher, Dirk Muller, James A. West, Nicholas F. Borrelli, Douglas C. Allan, and Karl W. Koch. Low-loss hollow-core silica/air photonic bandgap fibre. Nature, 424(6949):657-659, 082003.

[213] F. Benabid, J. C. Knight, G. Antonopoulos, and P. St. J. Russell. Stimulated raman scattering in hydrogen-filled hollow-core photonic crystal fiber. Science, 298(5592):399-402, 102002.

[214] Dimitre G. Ouzounov, Faisal R. Ahmad, Dirk Müller, Natesan Venkataraman, Michael T. Gallagher, Malcolm G. Thomas, John Silcox, Karl W. Koch, and Alexander L. Gaeta. Generation of megawatt optical solitons in hollow-core photonic band-gap fibers. Science, 301(5640):1702-1704, 092003.

[215] Saikat Ghosh, Jay E. Sharping, Dimitre G. Ouzounov, and Alexander L. Gaeta. Resonant optical interactions with molecules confined in photonic band-gap fibers. Physical Review Letters, 94(9):093902-, 032005.

[216] P. S. Light, F. Benabid, F. Couny, M. Maric, and A. N. Luiten. Electromagnetically induced transparency in rbfilled coated hollow-core photonic crystal fiber. Optics Letters, 32(10):1323-1325, 2007.

[217] T. Takekoshi and R. J. Knize. Optical guiding of atoms through a hollow-core photonic band-gap fiber. Physical Review Letters, 98(21):210404-, 052007.

[218] S Vorrath, S A Möller, P Windpassinger, K Bongs, and K Sengstock. Efficient guiding of cold atoms through a photonic band gap fiber. New Journal of Physics, 12(12):123015, 2010.

[219] Caleb A. Christensen, Sebastian Will, Michele Saba, Gyu-Boong Jo, Yong-Il Shin, Wolfgang Ketterle, and David Pritchard. Trapping of ultracold atoms in a hollow-core photonic crystal fiber. Physical Review A, 78(3):033429-, 092008.

[220] M. Bajcsy, S. Hofferberth, V. Balic, T. Peyronel, M. Hafezi, A. S. Zibrov, V. Vuletic, and M. D. Lukin. Efficient all-optical switching using slow light within a hollow fiber. Phys. Rev. Lett., 102:203902, May 2009.

[221] M. Bajcsy, S. Hofferberth, T. Peyronel, V. Balic, Q. Liang, A. S. Zibrov, V. Vuletic, and M. D. Lukin. Laser-cooled atoms inside a hollow-core photoniccrystal fiber. Physical Review A, 83(6):063830-, 062011.

[222] Limin Tong, Rafael R. Gattass, Jonathan B. Ashcom, Sailing He, Jingyi Lou, Mengyan Shen, Iva Maxwell, and Eric Mazur. Subwavelength-diameter silica wires for low-loss optical wave guiding. Nature, 426(6968):816819, 122003.

[223] K. P. Nayak, P. N. Melentiev, M. Morinaga, Fam Le Kien, V. I. Balykin, and K. Hakuta. Optical nanofiber as an efficient tool for manipulating and probing atomicfluorescence. Optics Express, 15(9):5431-5438, 2007.

[224] G. Sagué, E. Vetsch, W. Alt, D. Meschede, and A. Rauschenbeutel. Cold-atom physics using ultrathin optical fibers: Light-induced dipole forces and surface interactions. Physical Review Letters, 99(16):163602-, 102007.

[225] S. M. Spillane, G. S. Pati, K. Salit, M. Hall, P. Kumar, R. G. Beausoleil, and M. S. Shahriar. Observation of nonlinear optical interactions of ultralow levels of light in a tapered optical nanofiber embedded in a hot rubidium vapor. Physical Review Letters, 100(23):233602-, 062008.

[226] E. Vetsch, D. Reitz, G. Sagué, R. Schmidt, S. T. Dawkins, and A. Rauschenbeutel. Optical interface cre- 
ated by laser-cooled atoms trapped in the evanescent field surrounding an optical nanofiber. Physical Review Letters, 104(20):203603-, 052010.

[227] S. T. Dawkins, R. Mitsch, D. Reitz, E. Vetsch, and A. Rauschenbeutel. Dispersive optical interface based on nanofiber-trapped atoms. Physical Review Letters, 107(24):243601-, 122011.

[228] C-L Hung, S M Meenehan, D E Chang, O Painter, and H J Kimble. Trapped atoms in one-dimensional photonic crystals. New Journal of Physics, 15(8):083026, 2013.

[229] A. Goban, C. L. Hung, S. P. Yu, J. D. Hood, J. A. Muniz, J. H. Lee, M. J. Martin, A. C. McClung, K. S. Choi, D. E. Chang, O. Painter, and H. J. Kimble. Atomlight interactions in photonic crystals. Nat Commun, 5, 052014.

[230] D E Chang, L Jiang, A V Gorshkov, and H J Kimble. Cavity qed with atomic mirrors. New Journal of Physics, 14(6):063003, 2012.

[231] Douglas J. S., Habibian H., Hung C.-L., Gorshkov A. V., Kimble H. J., and Chang D. E. Quantum many-body models with cold atoms coupled to photonic crystals. Nat Photon, 9(5):326-331, 052015.

[232] Inbal Friedler, David Petrosyan, Michael Fleischhauer, and Gershon Kurizki. Long-range interactions and entanglement of slow single-photon pulses. Phys. Rev. A, 72:043803, Oct 2005.

[233] David Petrosyan and Michael Fleischhauer. Quantum information processing with single photons and atomic ensembles in microwave coplanar waveguide resonators. Phys. Rev. Lett., 100:170501, Apr 2008.

[234] M. Müller, I. Lesanovsky, H. Weimer, H. P. Büchler, and P. Zoller. Mesoscopic rydberg gate based on electromagnetically induced transparency. Phys. Rev. Lett., 102:170502, Apr 2009.

[235] Ephraim Shahmoon, Gershon Kurizki, Michael Fleischhauer, and David Petrosyan. Strongly interacting photons in hollow-core waveguides. Phys. Rev. A,
83:033806, Mar 2011.

[236] Alexey V. Gorshkov, Johannes Otterbach, Michael Fleischhauer, Thomas Pohl, and Mikhail D. Lukin. Photon-photon interactions via rydberg blockade. Phys. Rev. Lett., 107:133602, Sep 2011.

[237] Johannes Otterbach, Matthias Moos, Dominik Muth, and Michael Fleischhauer. Wigner crystallization of single photons in cold rydberg ensembles. Phys. Rev. Lett., 111:113001, Sep 2013.

[238] J. D. Pritchard, D. Maxwell, A. Gauguet, K. J. Weatherill, M. P. A. Jones, and C. S. Adams. Cooperative atom-light interaction in a blockaded rydberg ensemble. Phys. Rev. Lett., 105:193603, Nov 2010.

[239] Thibault Peyronel, Ofer Firstenberg, Qi-Yu Liang, Sebastian Hofferberth, Alexey V. Gorshkov, Thomas Pohl, Mikhail D. Lukin, and Vladan Vuletic. Quantum nonlinear optics with single photons enabled by strongly interacting atoms. Nature, 488(7409):57-60, 082012.

[240] Valentina Parigi, Erwan Bimbard, Jovica Stanojevic, Andrew J. Hilliard, Florence Nogrette, Rosa TualleBrouri, Alexei Ourjoumtsev, and Philippe Grangier. Observation and measurement of interaction-induced dispersive optical nonlinearities in an ensemble of cold rydberg atoms. Phys. Rev. Lett., 109:233602, Dec 2012.

[241] Y. O. Dudin and A. Kuzmich. Strongly interacting rydberg excitations of a cold atomic gas. Science, 336(6083):887-889, 2012.

[242] C. S. Hofmann, G. Günter, H. Schempp, M. Robert-de Saint-Vincent, M. Gärttner, J. Evers, S. Whitlock, and M. Weidemüller. Sub-poissonian statistics of rydberginteracting dark-state polaritons. Phys. Rev. Lett., 110:203601, May 2013.

[243] D. Maxwell, D. J. Szwer, D. Paredes-Barato, H. Busche, J. D. Pritchard, A. Gauguet, K. J. Weatherill, M. P. A. Jones, and C. S. Adams. Storage and control of optical photons using rydberg polaritons. Phys. Rev. Lett., 110:103001, Mar 2013. 